TOPOGRAPHIC PRECONDITIONING OF OPEN OCEAN DEEP CONVECTION

by

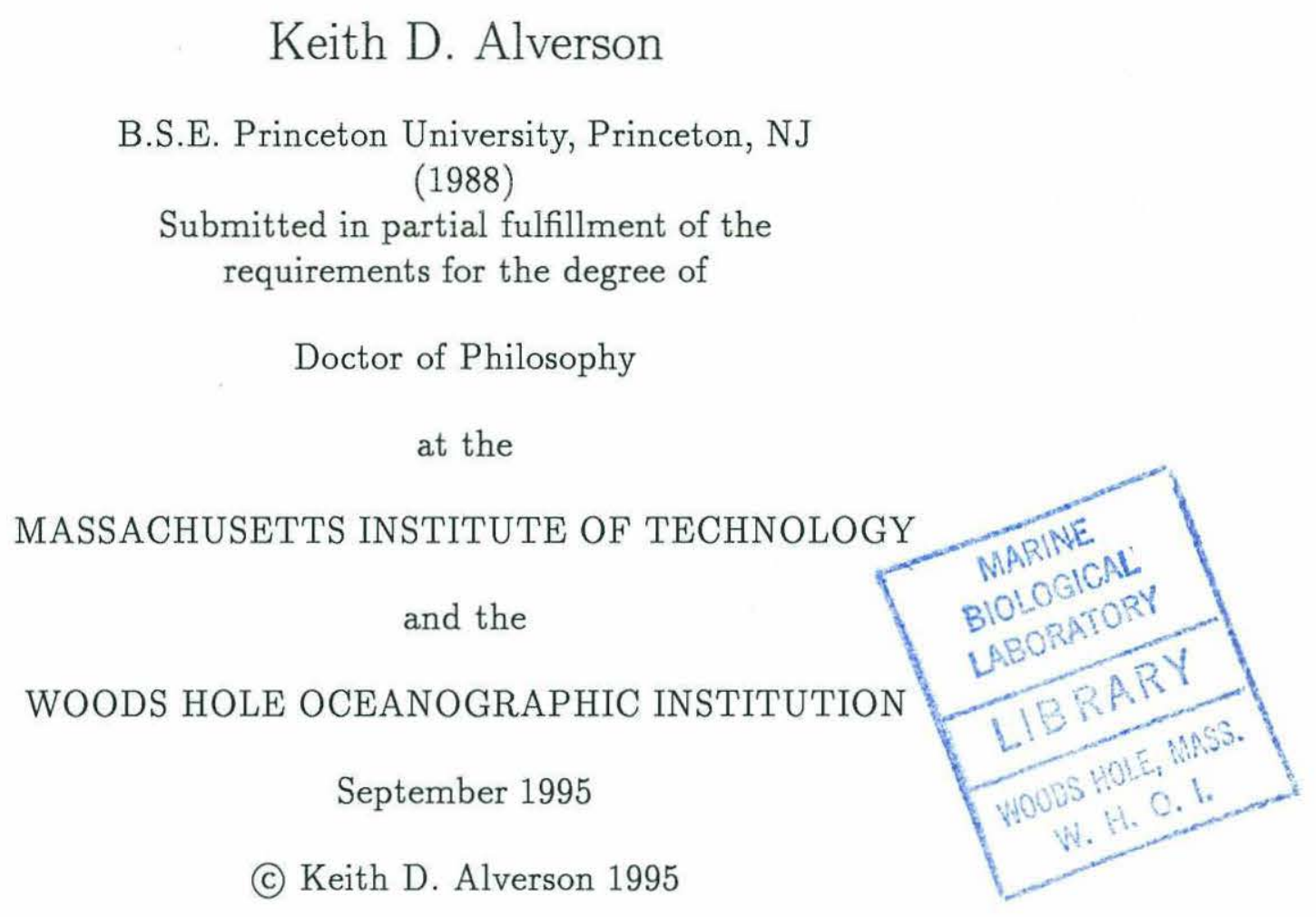

The author hereby grants to MIT and to WHOI permission to reproduce and to distribute copies of this thesis document in whole or in part.

Signature of Author ............................................... Joint Program in Physical Oceanography Massachusetts Institute of Technology Woods Hole Oceanographic Institution

September, 1995

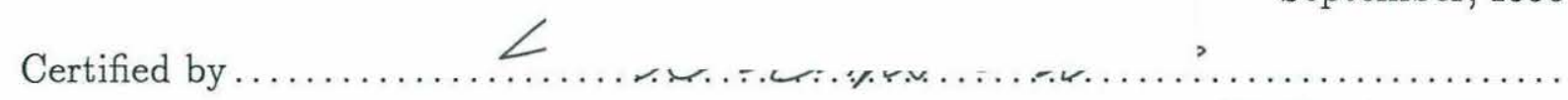
W. Brechner Owens

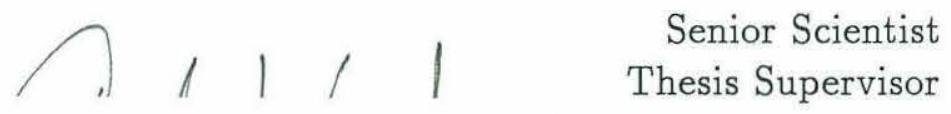

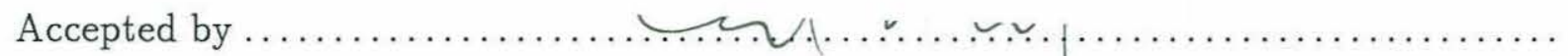

Carl Wunsch Chairman, Joint Committee for Physical Oceanography Massachusetts Institute of Technology Woods Hole Oceanographic Institution 


\title{
TOPOGRAPHIC PRECONDITIONING OF OPEN OCEAN DEEP CONVECTION
}

by

\author{
Keith D. Alverson
}

Submitted in partial fulfillment of the requirements for the degree of Doctor of Philosophy at the Massachusetts Institute of Technology and the Woods Hole Oceanographic Institution

September, 1995

\begin{abstract}
Evidence of enhanced oceanic convection over Maud Rise in the Weddell Sea indicates that bottom topography may play a role in selecting the location and scale of deep convecting oceanic chimneys below large scale atmospheric negative buoyancy forcing.

Topographic preconditioning of open ocean deep convection is studied using an idealized, three-dimensional, primitive-equation model. A barotropic mean flow impinges on an isolated Gaussian-shaped seamount in a stratified domain with uniform negative surface buoyancy forcing. A region of topographically trapped flow forms over the topography. When this "Taylor cap" is tall enough to interact with the surface mixed-layer, the local isolation from mean horizontal advection forms a conduit into the deep water. The convective penetration depth within this local region is significantly enhanced relative to ambient levels away from the seamount and to similar runs performed without bottom topography. The parameter dependencies for these preconditioning processes are investigated.

With uniform background stratification, the doming of isopycnals does not play a major role in the preconditioning process. However, when a surface intensified stratification is included, domed isopycnals associated with the Taylor cap circulation can also play a preconditioning role. In this case, the pycnocline is first ventilated over the seamount, leading to rapid convective deepening into the weakly stratified deep water. An analytical formula for one-dimensional, non-penetrative convection into an exponential stratification profile is derived and compares well with results from the numerical model.

Previous modeling studies have often parameterized the mehanism by which the horizontal scale of oceanographic chimneys is set through the use of disk-shaped surface forcing functions. Unlike in such experiments, topographically preconditioned chimneys are not prone to breakup by the growth of baroclinic instabilities. Instead, convection is generally shut down by horizontal fluxes of heat due to the mean flow across the temperature gradients of the chimney walls. The presence of the mean flow, which is neccessary in order for the topographic preconditioning to work, causes instabilities to be advected downstream faster than they can grow locally. These results suggest that
\end{abstract}


the role of baroclinic eddies in shutting down oceanographic convection is probably misrepresented in studies which parameterize the preconditioning mechanism, particularly if the preconditioning mechanism being parameterized is a topographic one.

Thesis Supervisor: W. Brechner Owens, Professor, Physical Oceanography Department Woods Hole Oceanographic Institution 


\section{Acknowledgements}

I have enjoyed my time as a graduate student at MIT and WHOI immensely. The years of learning which have culminated in this thesis (the ultimate sciolist credentials) were enjoyable primarily because of the wonderful people with whom I have interacted both socially and scientifically. Obviously I cannot do justice to everyone who has motivated, mentored, or moaned with me within the confines of this short space, but I will ramble on a bit in a generally thankful vein.

During the first two years of the program, I kept looking forward to the end of classes and exams in order to concentrate on research. Despite this palpably irrational desire, I did appreciate the opportunity to learn from two true masters of the art of pedogogy, Carl Wunsch and Joe Pedlosky. They introduced me to oceanography and taught me to think like a scientist. My fellow students learned the lecture material with ease, which allowed me to glean a few scraps second hand, especially from Sarah "it's trivial" Gille, Paul "think about it" Robbins, Ari "I haven't started yet" Epstein, Ed "Peary made it" Dever, Bing-of-the-secret-agenda, and Jim Goonson.

After that little bump in the road called the general exam I finally did manage to do a little research thanks to the help and support of my thesis committee: Mike Spall, Nelson Hogg, John Marshall, Dave Chapman and my advisor, Breck Owens. In addition, Glen Gawarkiewicz and Glenn Flierl had many helpful suggestions and were always happy to listen to my silly questions. I want to thank Dave Chapman, in particular, for taking far too much of his time to steer me through my initial encounters with the SPEM model and the problem of flow over topography as well as for reading every draft I gave him with a fine tooth comb. Breck was exactly the advisor I would choose if I could do it all over again. He gave me all the freedom I could ever ask in chosing a thesis topic, and oversaw the learning experience of tackling the problem with just the right amount of constructive criticism, and an underlying, unquestioning, support.

I would like to thank the MIT-WHOI Joint Program for the Paul Fye fellowship which covered my first year in graduate school as well as a generous allowance for books. Financial support for the thesis research was provided by NOAA grant number NA16RC0073 and NSF grant number OCE90-04864. Additional financial support was provided by the Office of Naval Research, Physical Oceanography Division under grant number N0001486-K-0751 as well as an AASERT fellowship, grant number N00014-89-J-1106.

Along the way I wasted an awful lot of time. And I would like to thank those who helped me in that endeavor as well:

oThe MIT athletic department for all the free ice time I could ever ask for.

- Lars Schade for always cycling faster than me so that I could draft.

- Chris Edwards for putting up with me as a volleyball partner.

-Richard "Flea" Wardle for those scintillating stories on bad hair days.

-Mom and Pats for the free weekend passes to God's country.

In the end though, Min Ku really did contribute the Max. 


\section{Contents}

$\begin{array}{ll}\text { Abstract } & 3\end{array}$

$\begin{array}{ll}\text { Acknowledgements } & 5\end{array}$

1 Introduction $\quad 9$

1.1 Open Ocean Convection in the Weddell Sea . . . . . . . . . . . . . . 11

1.1.1 Hydrographic Data . . . . . . . . . . . . . . . . . 11

1.1.2 Satellite Passive Microwave Sea Ice Concentration Data . . . . . . 17

1.2 Historical Overview . . . . . . . . . . . . . . . . . . . . . . . . . . . . . . . .

1.2 .1 Flow over Isolated Topography . . . . . . . . . . . . . . . . . 19

1.2 .2 Open Ocean Convection . . . . . . . . . . . . . . . . 21

1.2.3 Topographic Influences on Convection . . . . . . . . . . . . 24

1.3 Overview of the Thesis . . . . . . . . . . . . . . . 26

2 The Model $\quad 29$

2.1 The Numerical Model . . . . . . . . . . . . . . . . . . . . . . . 29

2.2 Model Configuration . . . . . . . . . . . . . . . . . . 33

2.3 Open Boundary Conditions . . . . . . . . . . . . . . . . . . . . . . . . . . . . 38

2.3 .1 Background . . . . . . . . . . . . . . . . . 38

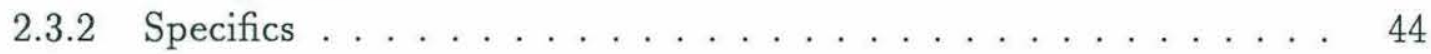

2.4 Surface Buoyancy Flux . . . . . . . . . . . . . . . . . . 46

3 Topographic Preconditioning of Open Ocean Convection 56

3.1 Introduction . . . . . . . . . . . . . . . 56

3.1 .1 One-Dimensional Analytic Limit. . . . . . . . . . . . . 56

3.1.2 Local Trapping as Topographic Preconditioning . . . . . . . . . . 58

3.2 Surface Mixed-Layer Depth . . . . . . . . . . . . . . . . . . . . 59

3.3 Taylor Cap Height . . . . . . . . . . . . . . . . . . . . . . . . . 65

3.3.1 Flow Reversal Diagnostic . . . . . . . . . . . . . . . 66

3.3.2 Passive Tracer Diagnostic . . . . . . . . . . . . . . . 70

3.3.3 Taylor Cap Height Parameter Dependencies . . . . . . . . . . . 73 
3.4 Taylor Cap Mixed-Layer Interaction . . . . . . . . . . . . . . . . . . 77

3.4 .1 Parameter dependencies . . . . . . . . . . . . . . . 81

3.5 Conclusions . . . . . . . . . . . . . . . . . . . . . . 87

4 Nonuniform Stratification $\quad 92$

4.1 Domed Isopycnals . . . . . . . . . . . . . . . . . . . . . 93

4.2 A One-Dimensional Analytic Model . . . . . . . . . . . . . . . . 96

4.3 A Simple Isopycnal Doming Experiment . . . . . . . . . . . . . . . . 103

4.4 Topographic Preconditioning Experiments . . . . . . . . . . . . . . . . . 104

4.5 Conclusions . . . . . . . . . . . . . . . . . . . . 110

5 Shutdown of Convective Deepening 112

5.1 Introduction . . . . . . . . . . . . . . . . . . . . . . . 112

5.2 Mechanisms . . . . . . . . . . . . . . . . . . . . . . 113

5.2.1 Disk-Cooling Experiment - D1 . . . . . . . . . . . . . 114

5.2.2 Stable Topographic Preconditioning Experiment - C4 . . . . . 117

5.2.3 Unstable Topographic Preconditioning Experiment - C6 . . . . . . 119

5.3 Baroclinic Instability of Convective Chimneys . . . . . . . . . . . 121

5.4 Conclusions . . . . . . . . . . . . . . . . . . . . . 131

6 Conclusions 135

6.1 Summary of the thesis . . . . . . . . . . . . . . . 135

6.2 Future Work . . . . . . . . . . . . . . . . . . . . . . . 140

$\begin{array}{lr}\text { References } & 143\end{array}$ 


\section{Chapter 1}

\section{Introduction}

In the Antarctic, during the winter, a vast region of ocean is subject to intense atmospheric cooling and salt rejection resulting from sea ice formation. Despite the widespread nature of this buoyancy loss, the resulting deep reaching open ocean convection occurs on a much smaller spatial scale. The wide disparity in the spatial scales of the atmospheric forcing and oceanic response can be explained if certain regions of the ocean have been preconditioned such that they preferentially convect. Killworth [1979], in a study of one convective feature, even hypothesized that in the absence of some mechanism for preselecting specific regions for convection, the entire Weddell gyre would be prone to overturning given the magnitude of typical winter forcing. Insofar as the basin scale

cyclonic circulation of the Weddell gyre represents a broad doming of isopycnals and an associated reduction in the heat content of the upper water column, the entire gyre is to some extent prone to convective overturning. In order to generate convection on the horizontal scales typically seen in the ocean, however, smaller regions of additional preconditioning must be superimposed within this larger gyre scale.

Preconditioning is used here as a general term for any dynamical processes in the ocean which can isolate a small, order 10 to 100 kilometer radius, patch of the ocean and allow it to convect to great depth relative to the ambient surface mixed-layer. This isolated deepening of the mixed-layer is often called a convective chimney. Thus, a 
preconditioning mechanism must select both a specific location as well as set a horizontal scale on which the chimney subsequently penetrates into the interior.

Because deep convection is forced at the ocean surface, far from the bottom boundary, the effect of bottom topography on the convective process is often ignored. However, convection tends to occur in regions of low ambient background stratification. In addition, oceanic flows are generally characterized by small Rossby number and are nearly inviscid. Given these conditions - low stratification, low Rossby number, and inviscid flow - the Taylor-Proudman theorem [eg. Pedlosky 1987] suggests that fluid motions in a rotating system should tend to be independent of the coordinate parallel to the axis of rotation. Thus, the influence of bottom topography can be expected to reach well up into the water column. As a result, bottom topography can be expected to influence the dynamics of surface driven convection, perhaps acting as a preconditioning mechanism. There are at least two means by which mean flow interacting with isolated topography can precondition the water column for convection: uplifted isopycnals associated with flow over the topography and isolation of topographically trapped fluid.

The purpose of this thesis is to examine how the interaction of oceanic mean flow with isolated bottom topography can influence the initial location of convecting chimneys, the depth to which they can be expected to penetrate, and the mechanisms by which the convection is shut down. In order to motivate the problem in terms of its application to the real ocean, section 1.1 provides an overview of some of the historical data indicative of open ocean convection in the Weddell Sea. I have chosen to concentrate on convection in the Weddell Sea because of its importance in setting the properties of Antarctic Bottom Water, which makes up the majority of the deep water in the world oceans. In addition, the scant evidence which does exist suggests that much of the open ocean convection which occurs in the Weddell Sea is concentrated in the vicinity of Maud Rise, a large isolated seamount with a horizontal scale of about 100 kilometers which rises roughly halfway into the water column from the otherwise flat and featureless 5000 meter deep Weddell Abyssal Plain. 
In section 1.2 , a short summary of the problem of flow over isolated topography is presented. Some of the important physical aspects of the problem of idealized flow over topography are introduced. In addition, an introduction to numerical modeling of oceanic convection is provided. This study has arisen as a direct amalgam of these two lines of research, in that it incorporates both flow over isolated topography and deep convection driven by surface buoyancy forcing. Finally, section 1.3 introduces this thesis research, a process oriented numerical modeling study, incorporating continuous stratification, tall topography and surface buoyancy forcing.

\subsection{Open Ocean Convection in the Weddell Sea}

Data indicative of open ocean convection in the Weddell Sea are primarily of two types: hydrographic data from ship based surveys and ice concentration data from satellite measurements. Most of the hydrographic data from the Weddell Sea have been taken in the summer season when retreat of the seasonal ice cover, combined with summer temperature and light conditions, allows relative ease of shipboard operations. However, modern high resolution CTD data are available for the Eastern Weddell from the Polarstern ANT V/2 cruise in the winter of 1986. Unfortunately, the 1986 winter cruise located no actively convecting chimneys.

\subsubsection{Hydrographic Data}

Evidence for open ocean convection appears in hydrographic data as anomalous regions of low stratification thought to be relict chimneys from the previous winter, and in changes in deep water characteristics following a winter of particularly strong, or weak, convection. 


\section{Relict Convective Chimneys}

Several summertime remnants of deep reaching convective chimneys, capped by warm, fresh, surface water due to ice melt and solar insolation, have been found in the Weddell not far to the west of Maud Rise. Gordon [1978] described a roughly $14 \mathrm{~km}$ radius eddy extending to at least 4000 meters depth with a surface cyclonic velocity signature of over $50 \mathrm{~cm} / \mathrm{s}$ located in the central Weddell Gyre. Temperature, salinity, oxygen and density sections through this convective feature are reproduced from Gordon in figure 1.1. The direction and magnitude of the mean flows in the region make it plausible that the chimney had been actively convecting the previous winter over Maud Rise, to the northeast of the location at which this remnant was found. Gordon suggested that open ocean convection in the vicinity of Maud Rise may produce a variety of Antarctic Bottom Water which can spread along isopycnals to replenish deep water outside of the Antarctic, whereas the denser bottom water produced along the continental margins may be topographically confined to some extent.

Foldvik et.al. [1985] reported two separate CTD casts showing anomalous thermohaline stratification similar to that found in the eddy observed by Gordon, and suggested that such deep convection phenomena may be quite common. Due to their wide station spacing, however, Foldvik was not able to determine the horizontal scale of these features. In 1983, Bersch [1988] found a region of convectively cooled and freshened water in the central Weddell reaching to at least 3000 meters depth, below which no data were available. He concluded, based on the same convective signal being seen in an XBT section 200 kilometers distant, that the horizontal scale of the convection was greater than $200 \mathrm{~km}$. Clearly, however, the possibility of two separate convective chimneys of smaller size cannot be excluded. A compendium of hydrographic evidence for convective chimneys, together with discussion of the possible evolution of convective features in the Weddell Sea has been published by Muench [1988]. 

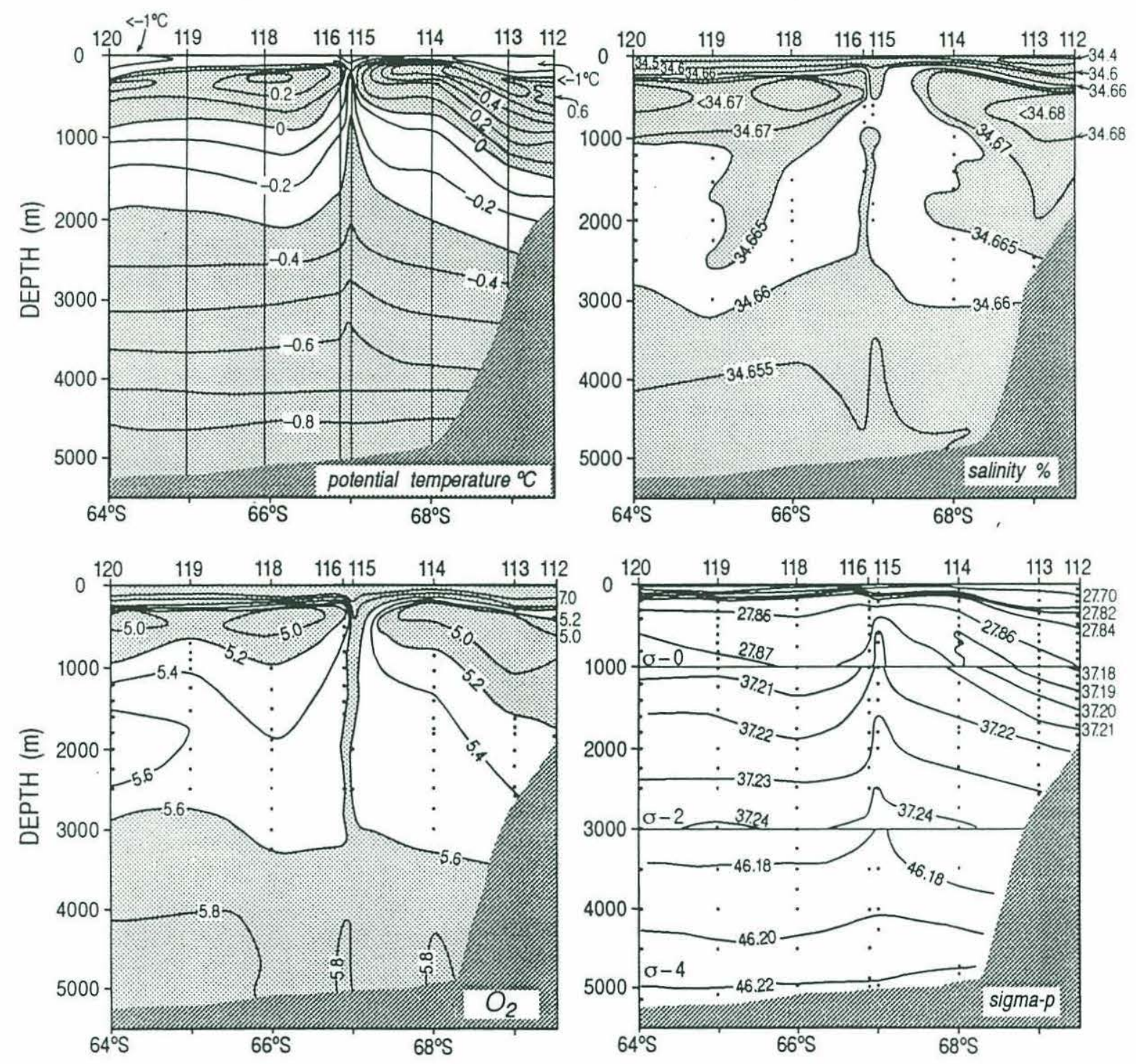

Figure 1.1: Along track temperature, salinity, oxygen and sigma-p showing the location and structure of a deep reaching convective feature west of Maud Rise. This figure is reproduced from Gordon [1978]. 


\section{Deep Water Temperature and Salinity Characteristics}

A high resolution wintertime hydrographic section running roughly north to south over Maud Rise is reproduced from Gordon and Huber [1990] in figure 1.2. The two panels show potential temperature and salinity along their cruise track. Maud Rise is clearly evident in the bottom topography. It is interesting to note that the warm salty water of North Atlantic origin lying between 500 and 1000 meters depth through most of the section is not present over Maud Rise. One likely explanation for this feature is that enhanced ventilation of the water over Maud Rise has partially homogenized the water column. In addition, a slight doming of isotherms is apparent over the topography.

Changes in the structure and properties of the deep water provide evidence for anomalously strong or weak convection. Gordon [1982], noted that the temperature of Weddell Deep Water was dramatically lower in 1977 than it was in 1973, with intense thermal alterations reaching to approximately 3000 meters depth. He attributed this cooling of the deep water to enhanced convective ventilation associated with the Weddell Polynya in the intervening years, which he estimates to have been between 1.6 and 7.7 Sverdrups depending primarily on whether the convection is assumed to have been occuring continuously, or primarily during the winter months. The Weddell Polynya, a large ice free region which occured in the Weddell sea for several consecutive years in the 1970's is discussed below.

In subsequent years a warming of the deep water has been observed. Foldvik et.al. [1985b] reported approximately $1{ }^{\circ} \mathrm{C}$ of warming in the core of the Weddell Deep Water between 1977 and 1979 which they attributed to the lack of Polynya activity during those years. It is important to note that these changes in deep water characteristics are only evidence of anomalous levels of open ocean convection, not the absolute rate of ventilation. The Weddell Polynya, because it occurred only for a few years, is ideally suited to this type of measurement. However, to the extent that a background level, or average amount, of convection occurs every year in the Weddell Sea, it will not show up as anomalous changes in the deep water characteristics. Rather, this open ocean 

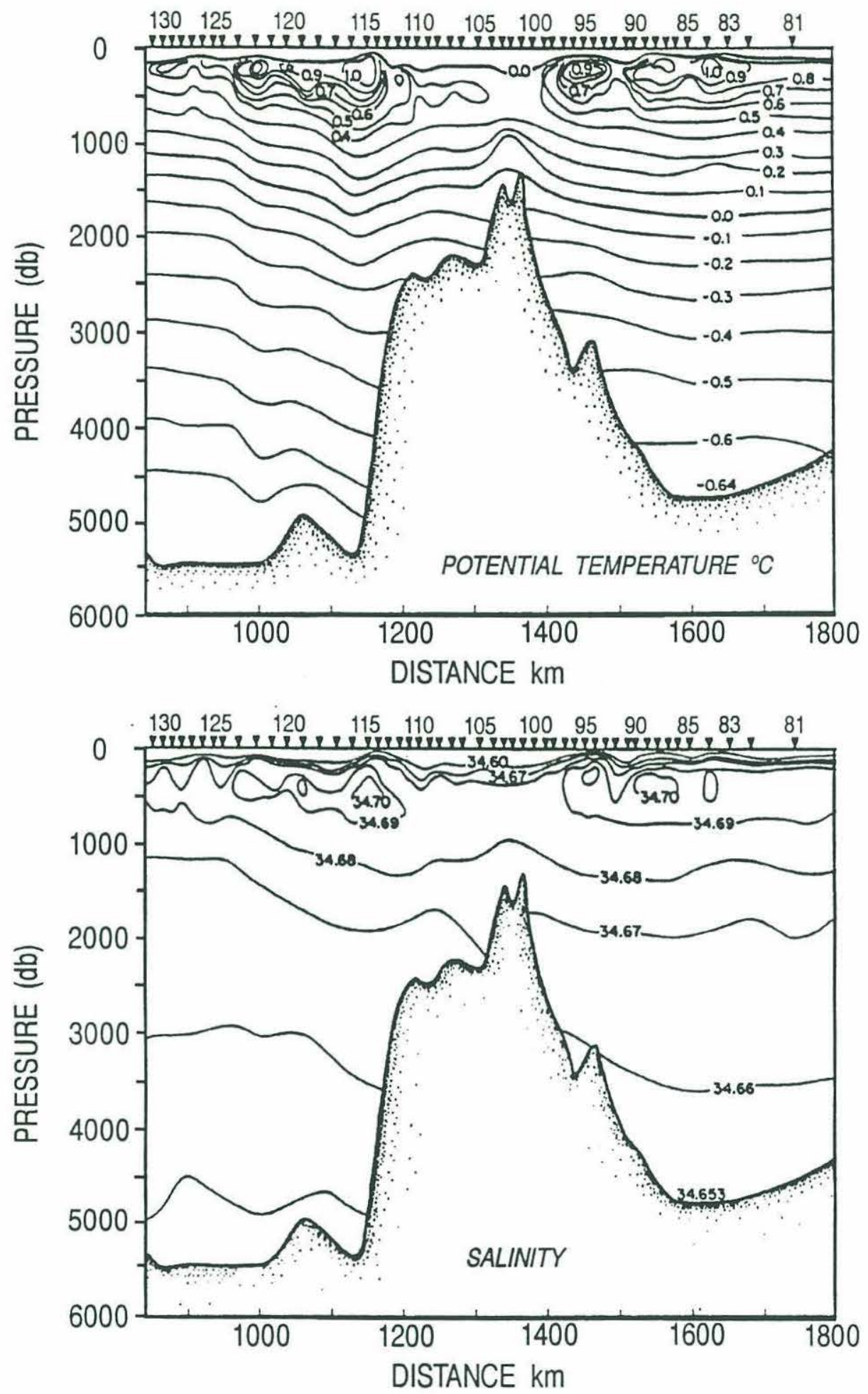

Figure 1.2: Wintertime sections of potential temperature and salinity over Maud Rise. This figure is reproduced from Gordon and Huber [1990]. 
ventilation is, in part, what sets the average Weddell Deep Water characteristics around which these anomalous measurements are defined.

\section{The Weddell Polynya}

Perhaps the most remarkable convective feature in the Weddell Sea was the magnificent Weddell Polynya. This immense region of open water was maintained through several winter seasons in the mid-1970's despite severe atmospheric cooling. After its initial formation in the vicinity of Maud Rise in 1974, the polynya drifted slowly westward with the mean circulation of the Weddell gyre over the following three seasons before vanishing. Although there remains evidence of significant convection in the vicinity of Maud Rise, the midwinter polynya has not been observed since 1976 .

Martinson et. al. [1981] used a two layer model to demonstrate that surface cooling and brine rejection from ice formation can lead to entrainment of warm salty water from depth with enough heat content to either melt back the initial ice cover or prohibit its formation all together, even given midwinter levels of atmospheric cooling. They suggested that bottom topography, although not explicitly included in their model, probably played a role in determining the initial location of the polynya by virtue of the locally uplifted pycnocline. Parkinson [1983] used a numerical model forced with realistic wind fields to suggest that the polynya may have been initiated by slow wind speeds in the centers of atmospheric lows, which would have reduced both sensible and latent heat loss to the atmosphere. However, given that her modelled polynya could not last the winter season, she concludes that heat fluxes from below due to oceanic convection are probably necessary to maintain the open water. Motoi et. al. [1987] used a one-dimensional, mixed-layer model to show that the major preconditioning factor was the presence of a salty mixed-layer in the preceding summer. Subject to the condition that atmospheric fresh water fluxes were less than $0.4 \mathrm{~m} /$ year, they conclude that the existence of the saline water was sufficient to ensure deep convection, without requiring either wind field anomalies or sea ice formation. 


\subsubsection{Satellite Passive Microwave Sea Ice Concentration Data}

Unlike hydrographic data, sea ice concentration information from satellite radar measurements is available continuously, with excellent temporal and spatial resolution. Indeed, it is only because of the advent of remote satellite measurements that the Weddell Polynya is known to have existed at all. On the negative side, satellite measurements are only capable of discerning the percentage of water covered by sea ice; they do not reveal anything about ice thickness (other than differentiating first year and multi-year ice which is of little use in the Weddell where nearly the entire ice cover is seasonal). In addition, the underlying isopycnal structure of the water column is opaque to electromagnetic sampling from satellite.

Sea ice concentration alone can be indicative of convective activity, however. If enough warm water from depth is brought to the surface, the sea ice can be partially or completely melted, leading to enhanced heat losses, further convection, and additional sea ice loss. The most magnificent evidence of such sea ice removal is of course the Weddell Polynya. False color satellite imagery of the entirely ice free polynya region and the rest of the Antarctic is available from NASA satellite passive radar data in Zwally et.al [1983]. Figure 1.3, reproduced from Zwally et.al, compares the September average ice cover in 1973, when no polynya was present, with the 1974 data when the polynya was situated directly over Maud Rise, just east of the Greenwich meridian, at 65 degrees south latitude.

Less obvious but more frequently occuring evidence of convection and the associated enhanced ocean heat fluxes is found in the consistent early spring meltback of the sea ice around Maud Rise. This early spring meltback is also visible in NASA false color images provided by Gloerson et.at. [1992]. Figure 1.4, derived from the Gloerson et.at. data, shows the sea ice distribution in December for several non-polynya years. Remarkably, nearly every spring the sea ice in the Weddell does not melt back from the ice edge, as one might expect given the latitudinal temperature gradient and mechanical forcing at the ice edge, but rather from the middle, in the vicinity of Maud Rise. This pattern of spring meltback can be taken as evidence suggestive of convection during the winter, 

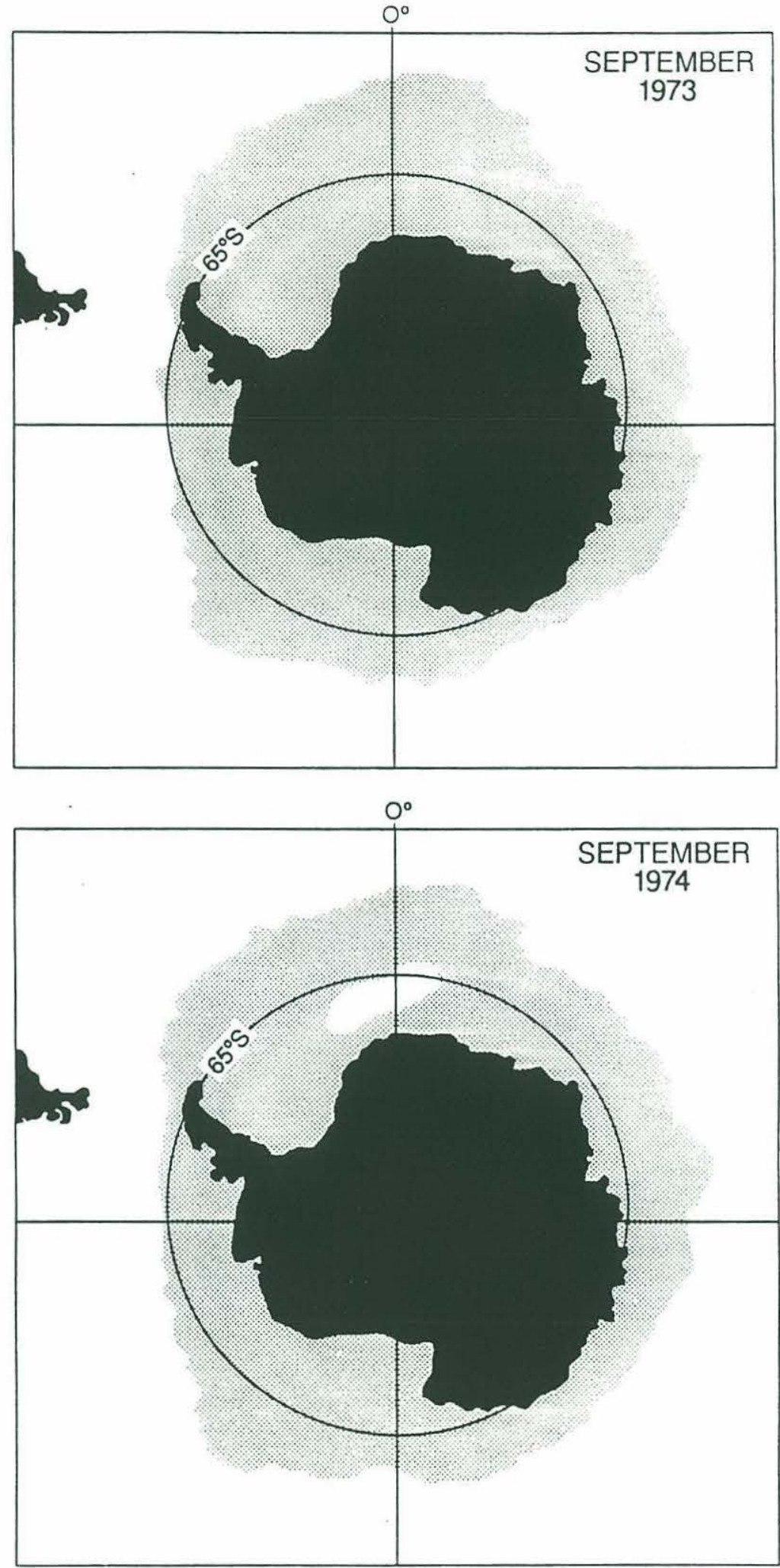

Figure 1.3: Wintertime (September) sea ice coverage in the Weddell Sea in 1973, a nonpolynya year, and 1974, when the polynya first appeared. This figure is adapted from false color satellite data in Zwally et.al [1983]. 
which would reduce ice thickness in the region over Maud Rise and thus allow for earlier removal in the spring, or, alternatively, as evidence of active convection in the early spring. In either case, a source of oceanic heat, as provided by deep reaching convection, is suggested by these data.

\subsection{Historical Overview}

\subsubsection{Flow over Isolated Topography}

The study of the effect of isolated bottom topography on rotating fluid motions has a long history. The seminal work by G.I. Taylor [1923] was primarily done with laboratory experiments in the context of unstratified flow. Taylor showed that when a mean flow impinges on a small bump on the bottom of a rotating tank, the fluid over the bump is trapped while the mean flow diverts around the obstacle. Such a column of topographically trapped fluid is now generally referred to as a Taylor column.

In the 1960's and 70's, a number of laboratory studies and analytical progress expanded on Taylor's original result. Hide $[1966,1968]$ considered the stratified problem and determined the critical topographic height required to trap fluid columns in a uniformly stratified, constant rotation system assuming small topography and Rossby number. In the stratified case, the steady-state flow past a seamount is characterized by both a local trapping of the fluid over the seamount and a doming of isopycnals, with stronger stratification increasing the degree to which these perturbations to the mean flow are bottom trapped. This region of trapped fluid is often called a Taylor cap, to distinguish it from the barotropic Taylor column. The effects of stratification together with background vertical shear were investigated by Hogg [1973]. Johnson [1978] looked at the effects of finite obstacle height and finite Rossby number. An excellent compendium of analytical work has been published by Thompson [1990, 1993].

As the investigation of flow over obstacles has become more sophisticated, numerical models have become increasingly useful. Huppert and Bryan [1976] used a numerical, 

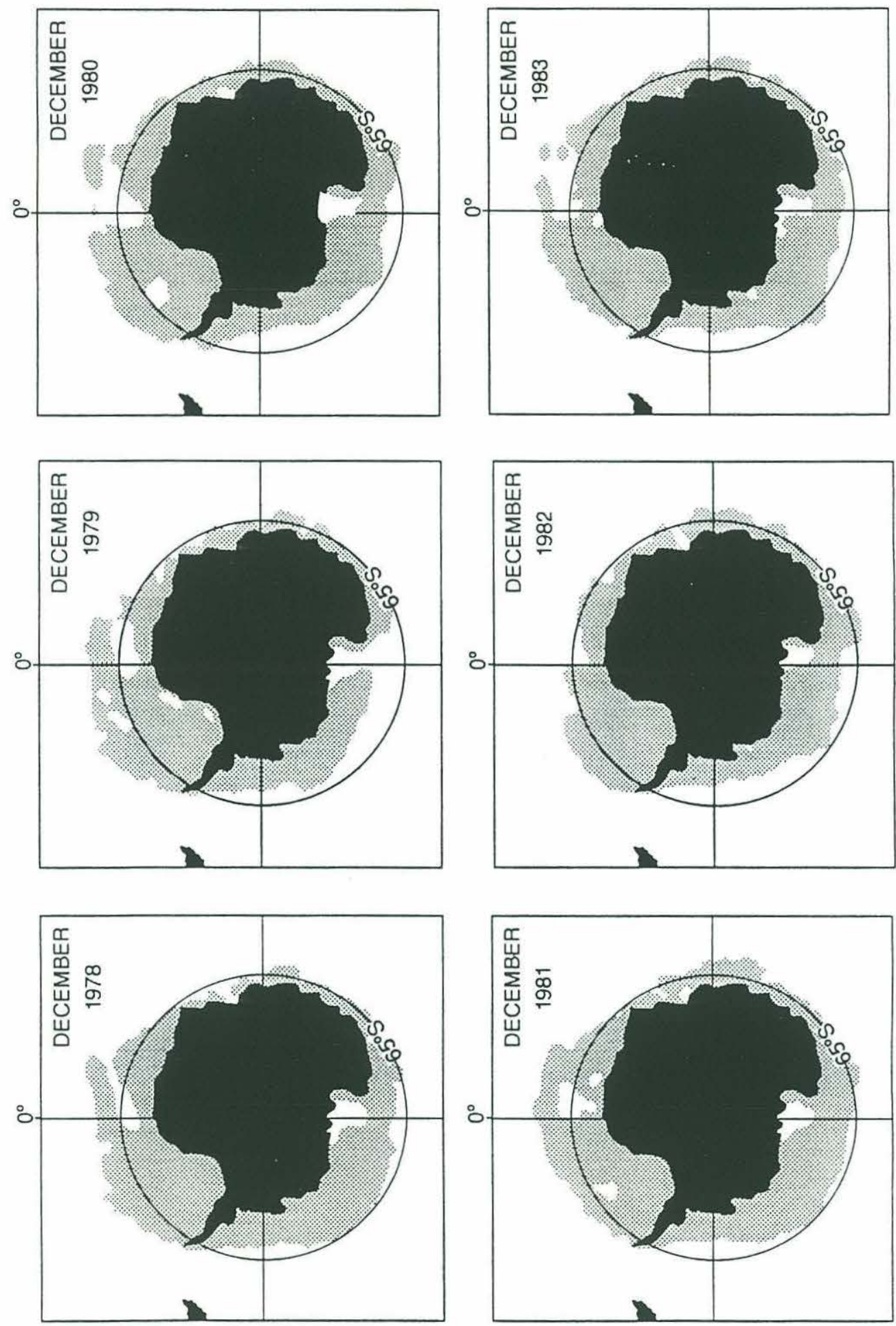

Figure 1.4: Late spring (December) sea ice coverage in the Weddell Sea for several nonpolynya years in the 1980's. This figure is adapted from false color satellite data in Gloersen et.al [1992]. 
primitive-equation model with periodic boundary conditions to investigate parameter dependencies for the stratified, uniformly rotating Taylor column spinup problem. As the incoming flow first impinges on the seamount, moving into shallower water, fluid columns are squeezed generating anticyclonic relative vorticity. Similarly, fluid columns moving off the seamount generate a cyclonic vorticity anomaly. These two vorticity anomalies are initially trapped on the flanks of the seamount and corotate. However, the mean flow, if it is strong enough relative to the eddy interactions, eventually advects the cyclonic anomaly downstream.

Open boundary conditions were incorporated in a barotropic, quasigeostrophic model by Verron and Le Provost [1985] in order to extend integration time and study eddy interactions and steady state oscillatory regimes without having information wrap around the domain and influence the upstream flow. The open boundary conditions allow a smooth transition from the transient problem described by Huppert and Bryan to a steady-state solution. Chapman and Haidvogel [1992] examined the spinup and steadystate behavior of the system outside of the restrictive quasigeostrophic limits using a primitive-equation model with open boundary conditions. Open boundary conditions are vital for examining the topographic effect on the convection problem because they allow the numerical integration to achieve a steady-state Taylor cap flow.

\subsubsection{Open Ocean Convection}

Deep reaching convective chimneys in the ocean, driven by buoyancy fluxes at the surface, occur in only a very few, small regions. One of the interesting aspects of the oceanic thermohaline circulation is the huge disparity between the limited size of these sinking regions and the much larger area thought to be characterized by slow upwelling. Killworth [1983] gives a summary of regions in the world ocean known to convect. He separates deep convection into two categories: (i) the "classic" sinking occurring on continental shelves and (ii) open ocean convection. This thesis is concerned only with the later. Killworth classifies three phases of open ocean convection, beginning with preconditioning, followed 
by violent mixing, and finally a sinking and spreading. Some historical perspective into the research that has been done to understand each of these phases in the convective process is given below.

\section{Preconditioning}

Early convection modelling was usually based on one-dimensional budgets which neglect the horizontal advection of heat. These models balance buoyancy loss through the upper surface with the difference between the buoyancy content of some initial profile and the homogenized water column after convection has taken place. Killworth [1979] used such a model with summertime hydrographic data from the Weddell Sea as an initial condition and realistic winter cooling rates. His surprising conclusion was that the entire Weddell gyre is susceptible to overturning and that a preconditioning mechanism must be operating in order for convection to occur only in limited geographic areas. There are many oceanographic phenomena which could potentially act as preconditioning mechanisms.

Remnants of previous convective chimneys are more likely to convect than surrounding water due to their reduced stratification; thus, these relict features may precondition convection. Killworth [1979] suggests that baroclinic instability in regions of zonal mean flow in the presence of meridional density gradients will generate cyclonic (as well as anticyclonic) eddies with an associated domed isopycnal structure. Domed isopycnals might tend to reduce the net heat content of the upper water column and thus serve as a preconditioning mechanism. Hakkinen [1988] suggests that upwelling at the ice edge due to differential Ekman drift occurs due to the sharp gradient in surface drag coefficient between ice covered and ice free regions as a preconditioning mechanism. Atmospheric forcing of surface divergence can remove insulating sea ice. The divergence leads to temporarily exposed open water and results in enormous local values of negative buoyancy flux as heat is lost to the atmosphere and new sea ice forms. All of these are plausible mechanisms for preselecting the location of deep penetrating convection. This thesis concentrates on the preconditioning effect of bottom topography. 


\section{Violent Mixing}

Large scale numerical models of the ocean have generally parameterized the effects of convection with a convective adjustment scheme in an otherwise hydrostatically stable model. However, high resolution three-dimensional primitive-equation modeling which attempts to resolve the convective processes in the ocean has been carried out with a nonhydrostatic model described in Brugge et.al. [1991]. The same model was used to examine the "violent mixing phase" of convection into a neutrally stratified ocean by Jones and Marshall [1993]. Jones and Marshall applied cooling to a circular region at the surface of an initially unstratified ocean. They found, in the parameter regime they feel is appropriate for oceanic convection, that the growth rate and depth reached by mesoscale convective chimneys is determined by a single non-dimensional parameter formed from the rate of rotation, ocean depth, and buoyancy flux. Klinger et.al. [in press] compared the use of traditional hydrostatic model physics with an associated convective adjustment scheme to the explicit inclusion of nonhydrostatic plume dynamics. They found that on the chimney scale and larger, hydrostatic models accurately reproduce the results of high resolution nonhydrostatic models.

It is worth noting that these disk-cooled convection studies, although informative, are forced in a rather unrealistic manner, with the surface cooling distribution setting the horizontal scale of the underlying chimney. In reality, atmospheric forcing is unlikely to occur on the same time or space scales as the resulting convective chimneys in the ocean. These studies rely on the implicit assumption that preconditioned regions exist in the real ocean which will determine the location and horizontal scale of the chimney without changing the other dynamical conclusions such as the depth to which the chimney penetrates. It is not clear that this assumption is justified.

\section{Sinking and Spreading}

After overturning and geostrophic adjustment of a convective chimney has occurred, lateral fluxes, perhaps due baroclinic eddies, distribute the newly formed dense water 
horizontally. Legg and Marshall [1993] used a heton model, initialized as a cloud of vortex pairs, analogous to individual plumes within a convective chimney, to model the breakup. The mass and energy fluxes from an initially uniform chimney in a linearly stratified fluid over an unstratified abyss were examined as a function of chimney size by Hermann and Owens [1993]. The breakup of chimneys in a neutrally stratified rotating fluid has also been examined in rotating tank experiments by Maxworthy and Naramosa [1994]. A useful review of both laboratory and numerical experiments is provided by Marshall, Whithead and Yates [in press].

Visbeck et.al. [in press] have extended the nonhydrostatic modeling work of Jones et.al. to include an initial background stratification. In this case, the convecting plume reaches a steady-state depth when baroclinic instability of the dense chimney allows sufficient horizontal eddy transports to balance the localized surface cooling. The final depth reached by the chimney is a function of the radius of the cooling disk, the magnitude of the surface forcing and the background stratification. Interestingly, this final depth of penetration is independent of the rotation parameter. The usefulness of a scaling argument which includes this arbitrary surface disk radius, rather than a horizontal length scale which might be important in real oceanic convection, seems questionable. In fact, in Chapter 5 of this thesis, one important result from the Visbeck et. al. study, namely the breakup of the chimney by baroclinic instability, is shown to be significantly altered when the chimney scale is determined by a topographic preconditioning mechanism rather than the scale of the surface forcing disk.

\subsubsection{Topographic Influences on Convection}

Although both flow over topography and surface forced convection have been studied in much detail, there have been few attempts to model them simultaneously. However, it has long been recognized that flow over topography can serve as a preconditioning mechanism for deep convection forced by buoyancy fluxes at the surface. The role of the Rhône Deep Sea Fan in preconditioning deep convection in the northwest Mediterranean 
Sea was discussed from an observational point of view by Swallow and Caston [1973] and modelled analytically, albeit without an explicit incorporation of surface forcing, by Hogg [1973b]. Hogg concluded that the topographically trapped, bottom intensified, anticyclonic flow serves to precondition the overlying fluid both because of the associated doming of isopycnals and by isolating the region from lateral fluxes of heat.

The problem of convection leading to ventilation of the deep waters around Maud Rise has received significant attention in recent years. Gordon and Huber [1990] discussed the hydrography around the rise in winter and postulated the presence of a Taylor column because of the significantly raised pycnocline. Ou [1991] studied how a Taylor column over Maud Rise may lead to enhanced convection. He used a highly idealized analytic model of two layer flow over a finite, right circular cylinder which did not include an explicit surface buoyancy forcing. Ou also pointed out that having the Taylor column "stacked" in both layers of his model was necessary for ventilation to reach below the pycnocline.

In summary, considerable evidence, from both hydrography and sea ice concentration data, suggests that open ocean convection occurs in the Weddell Sea, in the vicinity of Maud Rise. The numerical modeling of open ocean convection which has been done to date has set the scale and location of convective chimneys through the use of disk shaped surface forcing functions. This type of experiment assumes that some unmodeled oceanic mechanism sets the convective scale in the real ocean, but that this does not change the overall conclusions of the more idealistic modeling study. Previous modeling of flow over topography suggests that the influence of bottom topography could provide one such realistic mechanism for selecting the location and scale of oceanic convection. This thesis is an attempt to combine these two lines of research by incorporating surface buoyancy forcing in the context of flow over isolated bottom topography. 


\subsection{Overview of the Thesis}

This thesis is primarily a process oriented numerical modeling study of the role that isolated bottom topography can play in open ocean convection. The key parameters associated with this problem are a combination of those traditionally associated with convection and those associated with flow over topography: the surface buoyancy flux, the rate of planetary rotation, the background stratification, the mean upstream velocity, the length and height scales of the topography, and the ocean depth.

The primary motivation for this study is to understand the open ocean convection thought to occur in the vicinity of Maud Rise in the Weddell Sea. As a process oriented study, however, no attempt has been made to reproduce the exact topography, background stratification or surface fluxes in the Weddell Sea. As a consequence the study does not produce realistic estimates of bottom water formation rates around Maud Rise. Similarly, no attempt has been made to study, in a prognostic sense, likely changes in the deep water production due to environmental perturbations. However, the results do indicate the relative importance of the various physical parameters which describe this system, and can serve as a framework on which more detailed regional studies can be grounded. In addition, the results should be relevant to convection over topographic features in other parts of the world ocean such as the Mediterranean Sea and, perhaps, the Arctic or other regions in the Antarctic.

Chapter 2 is an introduction to the Semispectral Primitive-Equation Model (SPEM) developed by Haidvogel et.al. [1991] as well as the specific experimental setup used in this study. The chapter begins with a short description of the changes in the standard form of the hydrostatic, primitive-equations when they are expressed in sigma coordinates with a vertical spectral representation; the horizontal metrics associated with a stretched grid are also given. In addition, since open boundary conditions are vital to this problem, there is a short introduction to the problem of radiative boundary conditions in hyperbolic systems as well as the specific boundary conditions which I have employed. Finally, the 
incorporation of surface fluxes requires some form of mixed-layer dynamics. Thus, a constant depth slab mixed-layer which was developed to allow realistic incorporation of surface fluxes into the SPEM is described.

Chapter 3 details how mean flow, interacting with isolated bottom topography, can precondition a region to preferentially convect, relative to the surrounding area. Despite a uniform surface forcing, a convective chimney deepens significantly further over the flank of the topography than does the mixed-layer elsewhere in the domain. The essential mechanism for this topographic preconditioning is not domed isopycnals. Rather, it is primarily the local trapping of fluid in a Taylor cap, which cuts off the horizontal advection of heat by the mean flow. Away from the seamount the initial deepening of the mixed-layer is halted eventually by fluxes of heat associated with warmer fluid being advected into the model region from the upstream boundary; in the region of topographically trapped flow, however, the entire surface buoyancy loss is balanced by convective deepening. The parameter dependencies for this preconditioning process are investigated. Essentially, increasing mixed-layer depth and increasing Taylor cap height both tend to ensure that the effect of the topography is felt within the deepening mixedlayer, the key to chimney formation.

In chapter 4 the domed isopycnal paradigm for convective preconditioning is examined in some detail. Domed isopycnals do not appear to be important in the simplified topographic preconditioning problem examined in chapter 3. A one-dimensional, analytical calculation comparing constant background stratification to a somewhat more realistic surface intensified exponential profile demonstrates the reason for this phenomenon. Given a uniform stratification, doming of isopycnals actually tends to decrease local penetration of a convectively deepening mixed-layer. Thus, it is only when a pycnocline is present that domed isopycnals serve as a preconditioning mechanism. Given a surface intensification in the background stratification, the deep waters are first ventilated in the region of doming, and once this happens a convective chimney rapidly forms. A topographic preconditioning SPEM run similar to those in Chapter 3, but with an exponential 
background stratification, is described. The model run is in good agreement with the results of the analytical, one-dimensional calculation, indicating that once a pycnocline is present the doming of isopycnals over the topography, rather than the trapping of flow in a Taylor cap, serves as the primary preconditioning mechanism.

Finally, in Chapter 5, the shutdown of convective deepening in a topographically preconditioned chimney is compared to that observed in a chimney generated with a localized surface forcing. The presence of a mean flow around the topography has important effects with respect to the instability of the chimney. While chimneys generated under a disk of surface forcing are prone to baroclinic instability, the chimneys over topography show surprising stability. The reason for this stability appears to be that the growth rate of instabilities is an order of magnitude smaller than the timescale associated with flow past the chimney. As a consequence, instabilities are advected downstream away from the edge of the chimney faster than they can grow locally. 


\section{Chapter 2}

\section{The Model}

\subsection{The Numerical Model}

The dynamics associated with finite bottom topography combined with surface buoyancy forcing in a continuously stratified ocean have not been examined in much detail to date. The inherent nonlinearities in these dynamics suggest that a full primitive-equation model is necessary in order to capture the details of the relevant physics. I have chosen to work with the Semispectral Primitive-Equation Model (SPEM) version 3.2 developed by Haidvogel et. al. [1991]. In this chapter I describe this model and some minor modifications that I have made to it as well as the details of my particular application.

The SPEM solves the hydrostatic, Boussinesq, Navier-Stokes equations. These equations, consisting of momentum equations in three coordinate directions, the equation of continuity, the equation for the conservation of temperature and the equation of state, can be written:

$$
\begin{aligned}
\frac{\partial u}{\partial t}+\vec{u} \cdot \nabla u-f v & =-\frac{1}{\rho_{0}} \frac{\partial P}{\partial x}+F_{u} \\
\frac{\partial v}{\partial t}+\vec{u} \cdot \nabla v+f u & =-\frac{1}{\rho_{0}} \frac{\partial P}{\partial y}+F_{v} \\
\frac{\partial P}{\partial z} & =-\rho g
\end{aligned}
$$




$$
\begin{aligned}
\frac{\partial u}{\partial x}+\frac{\partial v}{\partial y}+\frac{\partial w}{\partial z} & =0 \\
\frac{\partial T}{\partial t}+\vec{u} \cdot \nabla T & =B+F_{T} \\
\rho & =\alpha T .
\end{aligned}
$$

Here $u$ and $v$ are the two horizontal components of the velocity vector, $\vec{u}, f$ is the Coriolis parameter, equal to half the local vertical component of the rate of rotation of the earth, $P$ is pressure, $\rho$ is the density, $\rho_{0}$ is a constant reference density, $g$ is the gravitational acceleration. The equation of state has been simplified by ignoring the effects of pressure and salinity, such that density is a linear function of temperature only, where $\alpha$ is the coefficient of thermal expansion. Horizontal diffusion is represented by the $F$ terms in the momentum and temperature equations. $B$ represents the surface heat flux. The terms representing horizontal diffusion $(F)$ and surface buoyancy flux $(B)$ are discussed in detail in sections 2.2 and 2.4 respectively.

Since the model is hydrostatic, any static instabilities must be removed using a simple convective adjustment scheme. The convective adjustment scheme operates after each timestep at each horizontal location in the domain. If the density at a given location in the vertical is greater than at the point immediately underneath, indicating a hydrostatically unstable state, the two grid cells are mixed and a new uniform density is applied to both levels. If any mixing is required, the routine is repeated until static stability has been achieved throughout the water column. The convective mixing scheme operates only on the density field. Thus, it does not directly change the momentum associated with each vertical level. However, given the rapid geostrophic adjustment to the changes in the density field, the mixing of density does, of course, effect the velocity.

The surface and bottom boundary conditions, in their most general form, include surface wind stress, bottom stress, and surface and bottom temperature flux conditions as well the kinematic conditions. In practice, however, I have set the surface and the 
bottom stress terms to zero:

$$
\left.\kappa_{u} \frac{\partial u}{\partial z}\right|_{z=0}=\left.\kappa_{u} \frac{\partial v}{\partial z}\right|_{z=0}=\left.\kappa_{u} \frac{\partial u}{\partial z}\right|_{z=-d}=\left.\kappa_{u} \frac{\partial v}{\partial z}\right|_{x=-d}=0
$$

where $d(x, y)$ is the depth of the domain. In addition, I have reformulated the manner in which surface heat flux is incorporated into the model. The surface cooling is incorporated in the B term in the heat conservation equation 2.1 rather than as a surface flux condition. The surface and bottom flux conditions are simply:

$$
\left.\kappa_{T} \frac{\partial T}{\partial z}\right|_{z=0}=\left.\kappa_{T} \frac{\partial T}{\partial z}\right|_{z=-d}=0 .
$$

The kinematic boundary condition at the surface is a rigid lid condition, which filters out surface gravity waves. The removal of these rapidly propagating surface gravity waves allows the use of a relatively long timestep. Finally, the bottom kinematic boundary condition is:

$$
\left.w\right|_{z=0}=\left.\left(u \frac{\partial d}{\partial x}+v \frac{\partial d}{\partial y}\right)\right|_{z=-d}=0 .
$$

In order to concentrate horizontal resolution where it is needed the SPEM allows for stretching of the horizontal grid. Given finite amplitude topography, there is a large horizontal variation in the local Rossby radius of deformation, which is proportional to the square root of the local depth. Hence, in order to minimize computational effort while resolving as well as possible the scale of the deformation radius, it is advantageous to incorporate a denser mesh of gridpoints over the seamount while sacrificing resolution in the deeper water. To this end, I make use of the curvilinear horizontal coordinate transformation incorporated in the SPEM.

In order to concentrate resolution at the center of the domain, where the seamount is located, I use a cosine shaped stretching of the coordinates:

$$
\begin{array}{r}
d \zeta=\left(1+S \cos \left(\frac{2 \pi i}{L-1}\right)\right) d x \\
d \eta=\left(1+S \cos \left(\frac{2 \pi j}{M-1}\right)\right) d y .
\end{array}
$$


Here $\mathrm{S}$ is a stretching factor set to $0.4, \mathrm{i}$ and $\mathrm{j}$ are the grid indices, and $\mathrm{L}$ and $\mathrm{M}$ are the total number of gridpoints in the $\mathrm{x}$ and $\mathrm{y}$ coordinate directions respectively. The nominal values of $\mathrm{dx}$ and dy are simply the domain size divided by total number of gridpoints in each horizontal direction.

One common problem encountered when bottom topography is incorporated into a numerical model is the difficulty in treatment of the bottom boundary condition. Zcoordinate finite difference models have a steplike approximation of the bottom topography which prohibits an easy implementation of nonzero vertical velocities required when there is flow across isobaths at the bottom of the model. The SPEM uses a stretched vertical coordinate which conforms to the bottom topography. This new vertical coordinate, commonly referred to as a "sigma" coordinate, varies from a value of 1 at the surface to -1 at the bottom. The coordinate transformation is simply:

$$
\sigma(x, y)=1+2\left(\frac{z}{d(x, y)}\right) .
$$

Together with this sigma transformation comes a new expression for the vertical velocity in sigma space, $\Omega$, given by:

$$
\Omega=\frac{d \sigma}{d t}=\frac{\partial \sigma}{\partial t}+u \frac{\partial \sigma}{\partial x}+v \frac{\partial \sigma}{\partial y}+w \frac{\partial \sigma}{\partial z} .
$$

Note that the sigma coordinate transformation greatly simplifies the bottom kinematic boundary condition in equation 2.4, which becomes $\Omega=0$ in the new coordinate frame. This simplification in the boundary conditions, however, comes at the expense of introducing some inaccuracy in the calculation of pressure gradient forces near steep topography and decreased numerical stability [McCalpin 1994]. In addition, there is some complication of the interior equations of motion. The hydrostatic, primitiveequations 2.1, after application of the horizontal and vertical transformations given by equations $2.5,2.6$, and 2.7, are the form of the dynamical equations solved by the SPEM.

The SPEM employs a spectral representation in the vertical. This means that rather than being represented on a grid, as with finite difference models, the model variables 
are expressed as the sum of a set of polynomial basis functions. A given model variable $\phi(\zeta, \eta, \sigma)$ can be expressed as:

$$
\phi(\zeta, \eta, \sigma)=\sum_{k} P_{k}(\sigma) \hat{\phi}_{k}(\zeta, \eta)
$$

where $P_{k}$ are a set of basis functions and $\hat{\phi}_{k}$ are their associated amplitudes. The default set of polynomial functions used by the SPEM are modified Chebyshev Polynomials, which provide enhanced resolution near the upper and lower boundaries of the domain relative to the interior. The model fields are actually stored at collocation points located at the maxima of the highest order polynomial mode rather than as modal amplitudes. Haidvogel et. al [1991] provide a detailed description of the SPEM.

\subsection{Model Configuration}

In order to examine the effectiveness of topographic preconditioning mechanisms, an experimental setup is required which allows investigation of mixed-layer deepening, the presence of a Taylor cap over isolated bottom topography, and lateral advection of heat due to a mean flow. The model domain I have chosen is a square channel 300 kilometers on a side with a depth, $H$, of 4000 meters. In the center of the domain is a Gaussian shaped bump characterized by a height, $h$, and horizontal e-folding scale, $L$. Thus, the depth as a function of $\mathrm{x}$ and $\mathrm{y}$ throughout the domain is:

$$
d(x, y)=H-h \exp \left(-\frac{\left(x-x_{0}\right)^{2}}{L^{2}}-\frac{\left(y-y_{\circ}\right)^{2}}{L^{2}}\right),
$$

where $\left(x_{0}, y_{0}\right)$ is the location of the seamount peak, in this case the center of the domain. The initial background stratification is linear with an associated buoyancy frequency, $N$. The variation of the coriolis parameter with latitude (the $\beta$ effect) is ignored. Thus, the entire domain rotates counterclockwise at a constant rate given by half the Coriolis parameter, $f$. Finally, the model is forced with a mean flow and surface cooling. The mean flow is an impulsively initiated barotropic zonal flow of speed $U$ forced by setting 


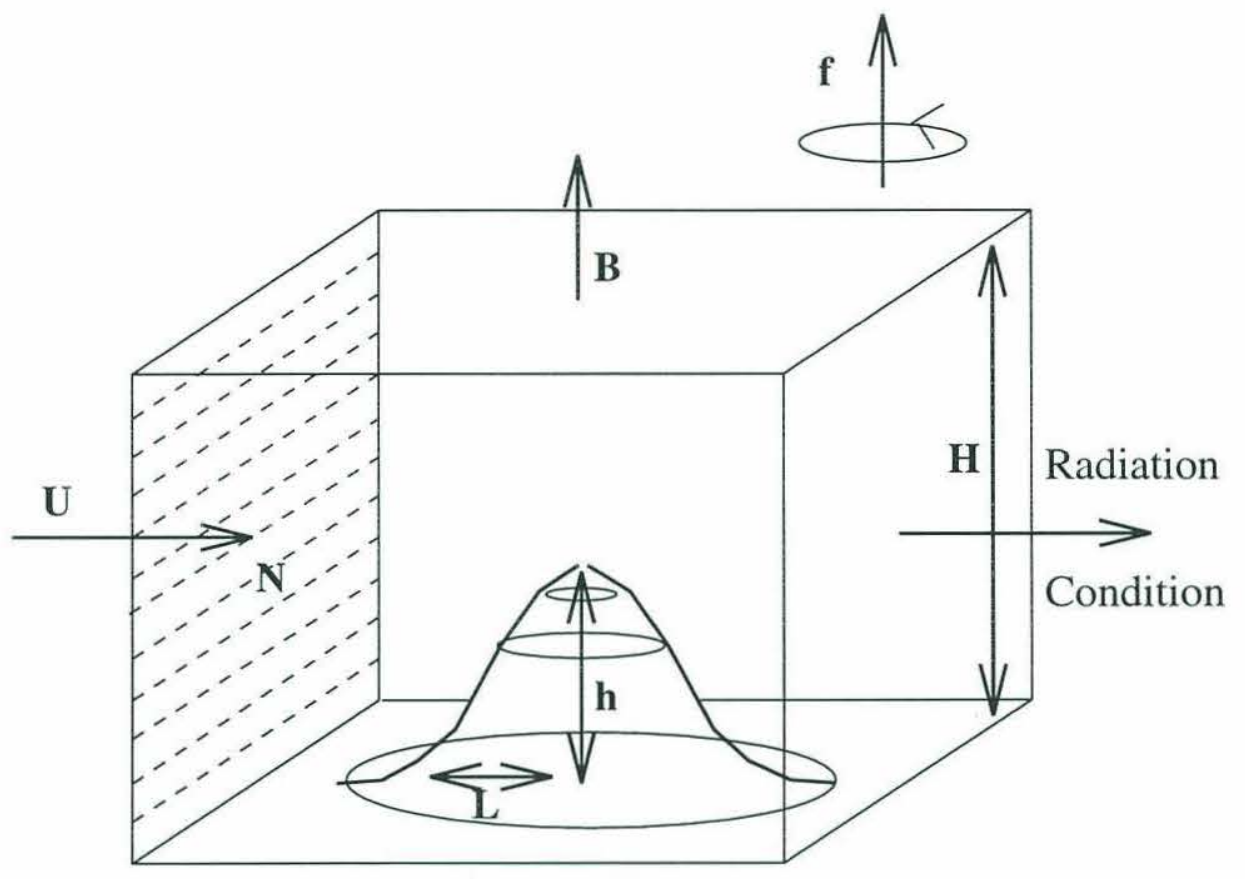

Figure 2.1: A schematic of the model domain and important physical parameters governing the flow: the characteristic scales of the seamount, $h$ and $L$, the buoyancy forcing, $B$, the mean flow velocity, $U$, the Coriolis parameter, $f$, the buoyancy frequency of the linear background stratification, $N$, and the ocean depth $H$.

streamfunction values on the inflow and sidewalls of the channel. The outflow is an open, radiative condition, discussed in more detail in section 2.3. A schematic of the model domain, including all of the physical parameters is shown in figure 2.1.

As can be seen from the extensive list of dimensional parameters needed to define the forcing and the domain itself, the dynamics of the flow are quite complex. The list of seven physical parameters: $h, L, H, U, N, f$, and $B$ is unfortunately quite long. Since all of the dimensional parameters can be expressed in terms of two fundamental units, time and space, the Buckingham-Pi theorem [Buckingham 1914] states that the number 


\begin{tabular}{|l|c|c|}
\hline & symbol & definition \\
\hline Parameter & $\mathrm{R}$ & $U / f L$ \\
Rtratification Parameter & $\mathrm{E}$ & $N / f$ \\
Fractional Height & $\delta$ & $h / H$ \\
Aspect Ratio & $\Delta$ & $H / L$ \\
Convective Rossby Number & $R_{N}$ & $\left(B / f^{3} H^{2}\right)^{1 / 2}$ \\
\hline
\end{tabular}

Table 2.1: Non-dimensional parameters important for governing Taylor cap formation and surface cooling.

of parameters can be reduced by two through an arbitrary choice of non-redundant dimensionless parameters. Although any set of non-dimensional combinations of the dimensional parameters is theoretically able to determine the system, I have chosen five dynamically relevant numbers, for convenience and recognizability, the Rossby Number, stratification parameter, fractional height, aspect ratio, and convective Rossby Number. These parameters are listed in Table 2.1.

The domain is modeled with 14 vertical polynomial modes and with 50 gridpoints in each horizontal direction. The coordinate stretching described by equation 2.5 results in a minimum horizontal resolution of 8.7 kilometers near the domain edges and a maximum of $3.7 \mathrm{~km}$ at the center of the domain. The stretched horizontal grid is shown in figure 2.2.

Subgridscale processes are parameterized by the diffusion terms, $F_{u}, F_{v}$ and $F_{T}$, in the horizontal momentum and temperature equations 2.1. I have chosen to represent diffusion with biharmonic mixing coefficients rather than the more traditional laplacian formulation primarily in an attempt to ensure numerical stability within the widest possible range of parameter space. In all of the runs, the diffusivity is kept as small as possible in the hope of closely approximating inviscid dynamics. Unfortunately, the centered difference scheme used by the SPEM is prone to growing numerical instabilities in calculation of horizontal derivitives, especially during spinup of the Taylor cap, when strong property gradients form over the topography. A small frictional smoothing of the gradients is one way of avoiding numerical, unrealistic strengthening of these fronts. The 


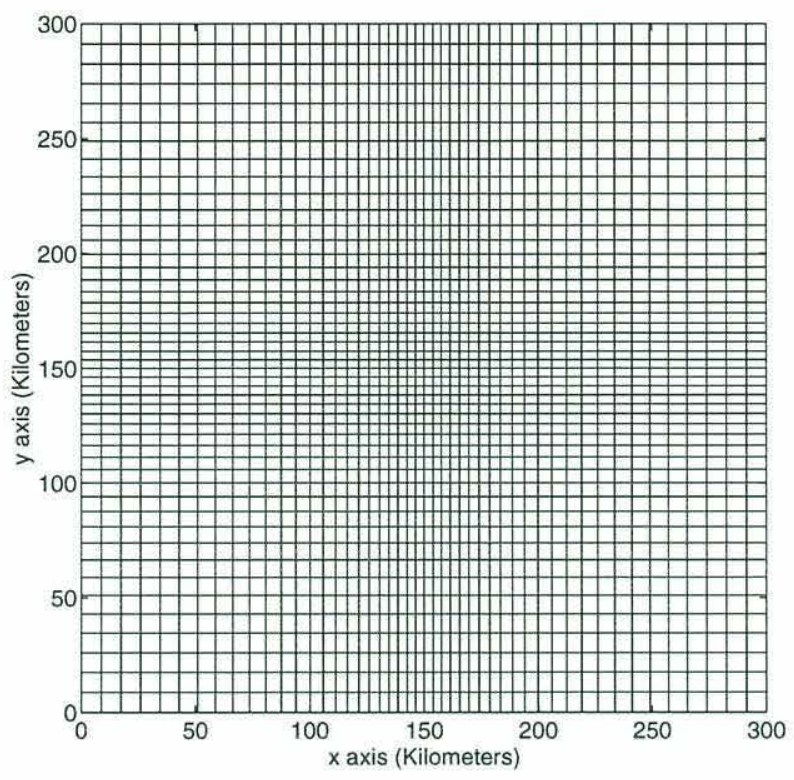

Figure 2.2: The horizontal grid with a stretching factor of 0.4 .

biharmonic mixing is applied along sigma surfaces, such that $F_{u}=\kappa_{u} \nabla^{4} u, F_{v}=\kappa_{u} \nabla^{4} v$, and $F_{T}=\kappa_{T} \nabla^{4} T$, using values of $4 \times 10^{9} 5 \times 10^{9}$ and for $\kappa_{T}$ and $\kappa_{u}$ respectively.

The mean flow forcing leads to the well studied spinup of a stratified Taylor cap. A series of horizontal density slices at 2000 meters depth during this spinup process is shown in figure 2.3 for one particular run (S2). This run is forced with a Rossby Number of 0.08 , an initial background stratification parameter of 5.556, a seamount fractional height of 0.5 , and an aspect ratio of 0.16 .

As the incoming flow first impinges on the seamount, moving into shallower water, fluid columns are squeezed generating anticyclonic relative vorticity. Similarly, fluid columns moving off the seamount are spun up in a cyclonic vorticity anomaly. These two vorticity anomalies are initially trapped on the flanks of the seamount and co-rotate. However, the mean flow, if it is strong enough relative to the eddy interactions, eventually advects the cyclonic anomaly downstream. The entire spinup process takes on the order of an advective timescale, $L_{x} / U$, to occur. In this case $L_{x}$, the length of the domain, is 300 kilometers, and the mean flow, $U$, is $20 \mathrm{~cm} / \mathrm{s}$, giving a spinup time of approximately 17 days. 

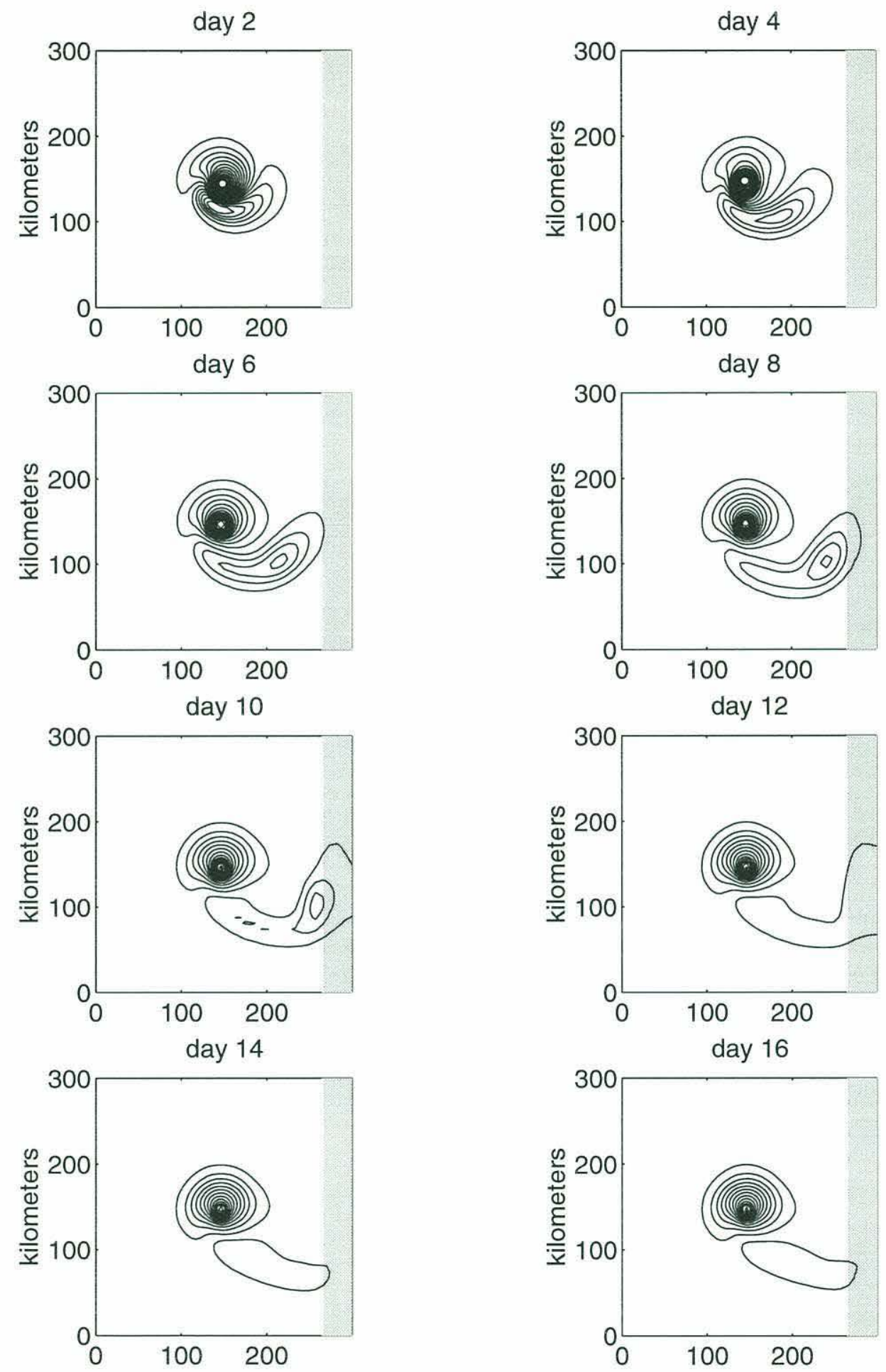

Figure 2.3: Temperature contours at 2000 meters for a Taylor cap spinup with an Orlanski open boundary condition and an associated sponge layer. The sponge layer is shaded. 
Once a Taylor cap is spun up over the topography, the ensuing steady-state is used as an initial condition for the convection experiments. Surface forcing is then applied as a negative buoyancy flux, $B$, applied uniformly to the upper boundary. The steadystate Taylor cap which serves as an initial condition for the convection experiments is marked by a region of trapped fluid over the seamount flank. Although the specifics of the flow field are of course dependent on the exact parameters of a given run, it is generally characterized by a doming of isopycnals over the topography. Associated with this doming is an anticyclonic relative vorticity anomaly and a deflection of the mean flow.

Figure 2.4 shows some of the fields for run S2 after 20 days of integration, once a steady-state has been achieved. The upper left panel shows the density field at 2000 meters in the same format as the slices shown for the spinup of this run in figure 2.3. The upper right hand figure shows the velocity field at 2000 meters depth. In each of the upper panels the underlying topography is shaded. The upper left panel shows a Y-Z density through the center of the domain, showing the doming of isopycnals. Finally, the lower right hand figure shows an $\mathrm{X}-\mathrm{Z}$ density slice through the center of the domain. In this slice the doming of isopycnals is displaced somewhat to the right, looking upstream, as expected given the velocity field is in thermal wind balance. The details of this and other steady-state Taylor cap runs, used as initial conditions for the convection experiments, are described in Chapter 3.

\subsection{Open Boundary Conditions}

\subsubsection{Background}

The initial condition required for the standard cooling experiments, is a steady-state flow over and around a seamount. In order to model such a steady-state, a working open boundary exit condition is absolutely vital. Without an open exit, eddies such as the cyclone shed during the spinup process could not leave the computational domain. 

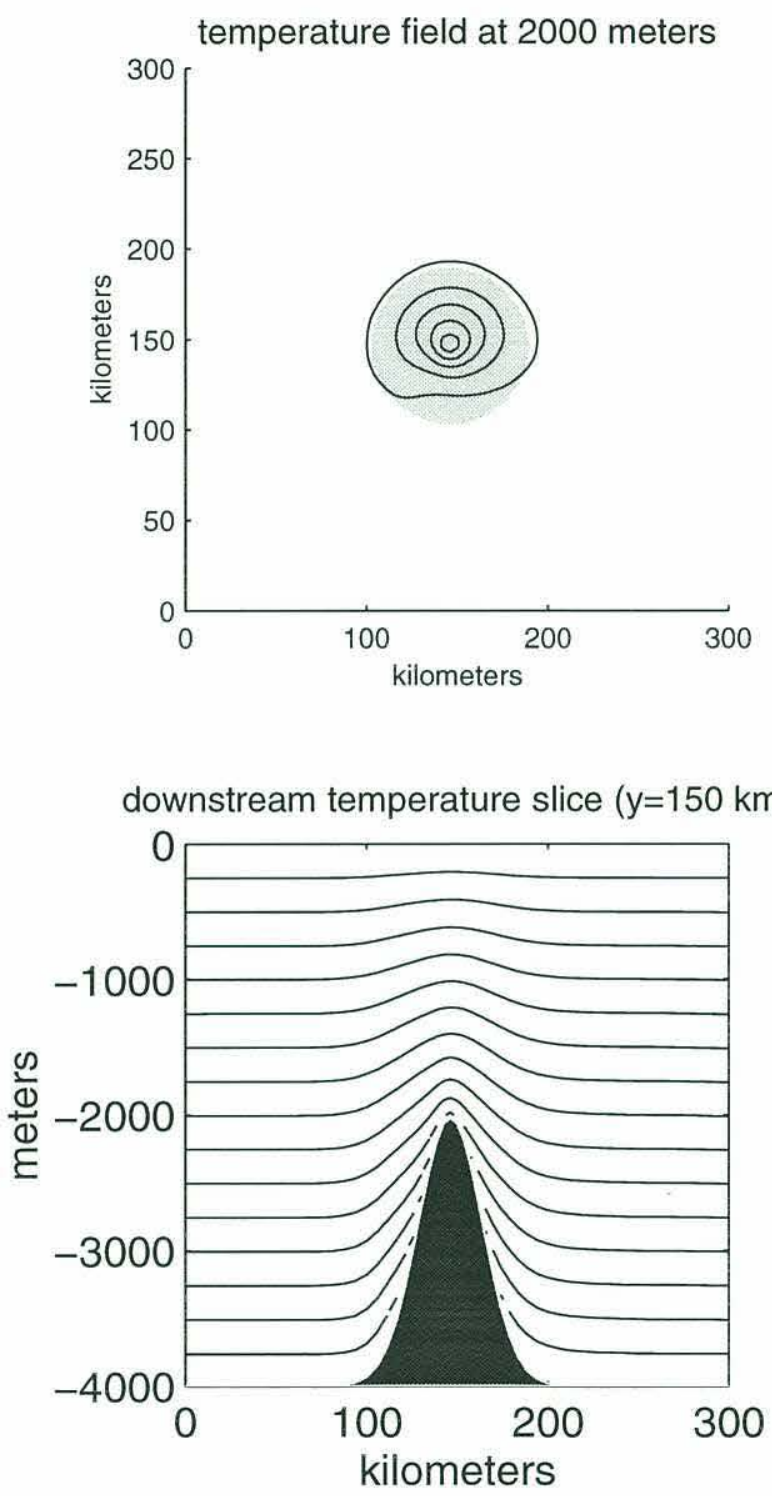
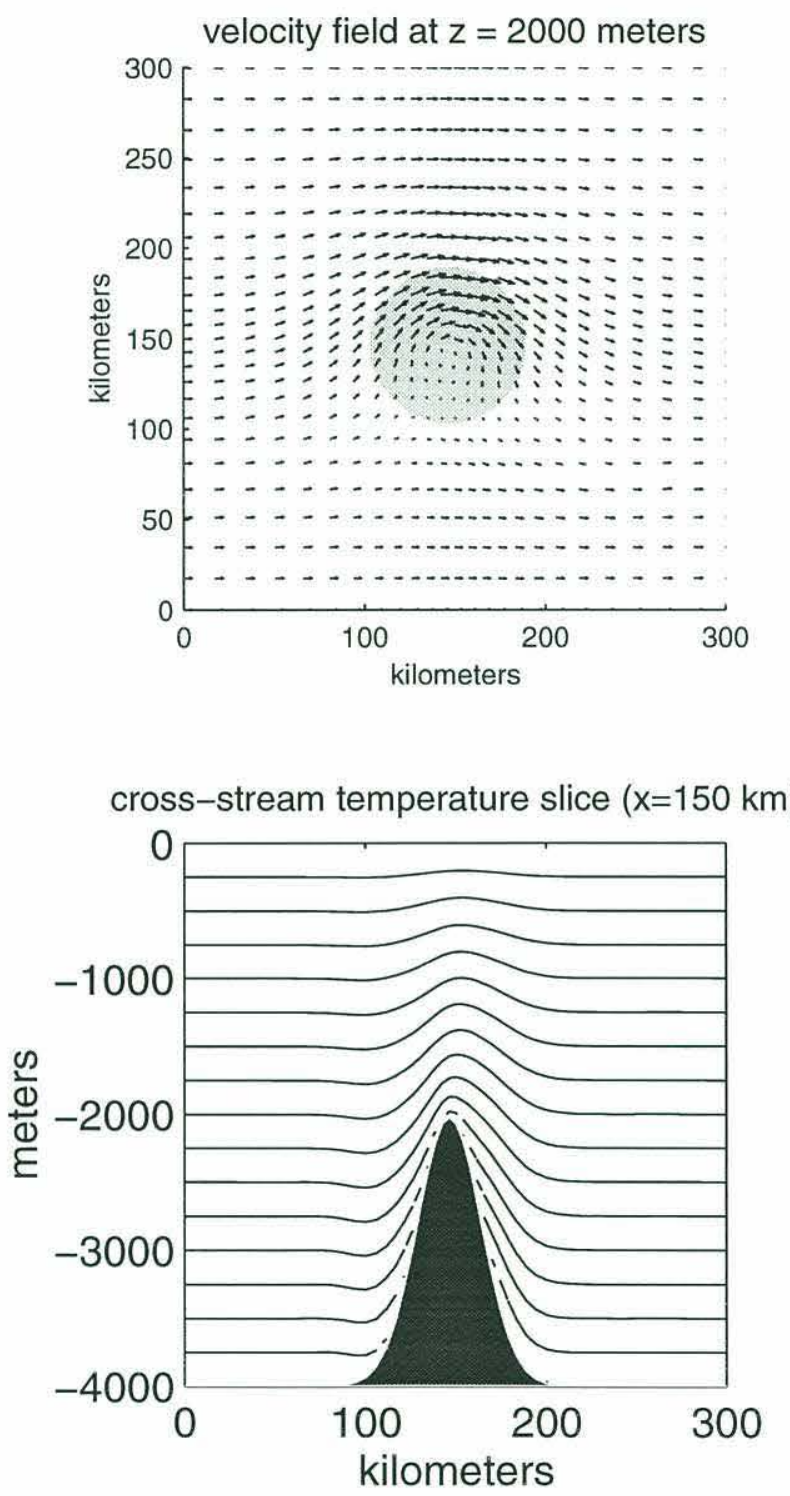

Figure 2.4: Steady-state property distributions for run S2 after 20 days of integration, once a steady-state has been achieved. The shading indicates the location of the seamount. 
Much of the early Taylor column modeling work concentrates on the spinup problem and, as a consequence, periodic boundary conditions are generally employed [Huppert and Bryan 1975, Smith 1991]. However open boundary conditions have been developed in the quasigeostrophic context [Verron and LeProvost 1985, Verron 1986] and, more recently, with a full primitive-equation model [Chapman and Haidvogel 1992].

The problem of how to incorporate open boundaries into numerical models has been the object of considerable study. In general, finite computer resources combined with a desire for high spatial and temporal resolution of relevant dynamics make it advantageous to choose boundaries to the computational domain other than the physical basin walls. Eventhough considerable effort is required to formulate a numerical boundary condition in a location where no physical boundary exists, this effort can be rewarded by allowing modeling efforts which would otherwise be unfeasible due to inadequate computer resources. There is a wide variety of problems in oceanography for which development of open boundary conditions is desirable, and has been attempted. Some examples include coastal modelling, flow around isolated obstacles and regional process studies. This study falls within the realm of both of the latter two examples.

The first question which must be answered when contemplating the incorporation of a boundary condition is whether it leads to a problem which is mathematically wellposed, in the sense of having a unique solution. In addition, when the mathematical condition does not coincide with any physical boundary in the system, there is the further complication that the problem could be physically ill-posed, in the sense that the interior solution is affected by the presence of this boundary in a way which does not reflect any processes inherent to the physical system being modeled.

As an example, consider the homogeneous one-dimensional wave equation:

$$
\frac{\partial^{2} u}{\partial t^{2}}+C \frac{\partial^{2} u}{\partial x^{2}}=0
$$

The equation is hyperbolic, with characteristics given by: 


$$
\xi=x-C t \text { and } \eta=x+C t .
$$

Waves propagating toward the right from a source at $x=0$ travel along the characteristics. A perfect open boundary condition at, say, $x=L$ would be one which absorbs all of this incident energy with zero reflection or generation of energy propagating back into the domain, toward the left. For this simple one-dimensional case such a boundary condition is easy to construct, and is given by:

$$
\frac{\partial u}{\partial t}=\left.C \frac{\partial u}{\partial x}\right|_{x=L}
$$

This boundary condition is commonly referred to as a Sommerfeld radiation condition. Note that the characteristic for this condition is $x-C t=$ constant, which exactly matches the characteristics incident on the boundary from the interior. Thus, the boundary will perfectly absorb all energy incident upon it from the interior without reflecting or generating energy.

Unfortunately, the extension of this type of radiation condition to more than onedimension and to dispersive systems in which the wave propagation speed is not constant is not straightforward. In multi-dimensional systems it becomes necessary for the boundary condition 2.12 , to be formulated in terms of the component of the wave phase velocity incident normal to the boundary, a condition which is trivially met in the onedimensional case. The extension to dispersive systems is more difficult. Bennett [1975] has constructed exact radiation conditions for certain dispersive wave systems including barotropic Rossby waves and non-hydrostatic internal gravity waves and finds that they require weighted information from the entire space and time domain of the problem. As he points out, numerical implementation of such a boundary condition "would require computer storage approaching that which one is trying to avoid by the introduction of open boundaries."

In addition, the presence of a mean flow, some component of which is incident on the 
open boundary, further complicates the issue by altering the speed at which anomalies propagate, and in some cases altering the number of conditions which must be prescribed on a given boundary in order that the problem remain mathematically well-posed. In order to examine this particular difficulty it is useful to consider a second, slightly more complicated system. The non-rotating, one-dimensional, linearized, shallow water equations for flow in an open channel are:

$$
\begin{array}{r}
\frac{\partial u}{\partial t}+U_{0} \frac{\partial u}{\partial x}=-g \frac{\partial h}{\partial x} \\
\frac{\partial h}{\partial t}+U_{0} \frac{\partial h}{\partial x}+H \frac{\partial u}{\partial x}=0 .
\end{array}
$$

The equations have been linearized, for simplicity, around a mean state velocity and depth. The characteristics for this system are:

$$
\xi=x+\left(U_{0}+C\right) t \text { and } \eta=x+\left(U_{0}-C\right) t,
$$

where $C$ is the shallow water gravity wave speed $\sqrt{g H}$.

In this system, the exit condition at $x=L$ is dependent on the magnitude of $U_{0}$ relative to $C$. If $U_{0}>C$ then the flow is supercritical and any information prescribed at the exit cannot propagate back upstream, against the flow, and affect the interior domain. Consequently, the height field at the exit can only be prescribed as a meaningful boundary condition leading to a well-posed problem in the case of subcritical flow.

The schematic representation of the characteristics shown in figure 2.5 helps to visualize the different exit conditions required for supercritical vs. subcritical flow. In both cases there are two characteristics emanating from the $t=0$ boundary of phase space, indicating that 2 pieces of information, $\mathrm{u}$ and $\mathrm{h}$, must be supplied as initial conditions. In the case of supercritical flow, two sets of characteristics also emanate from the entrance boundary at $x=-L$, whereas none emanate from the exit. Thus, in order to ensure a unique solution two boundary conditions must be supplied at the entrance while none may be supplied at the exit. Physically, this means that the interior flow is entirely deter- 

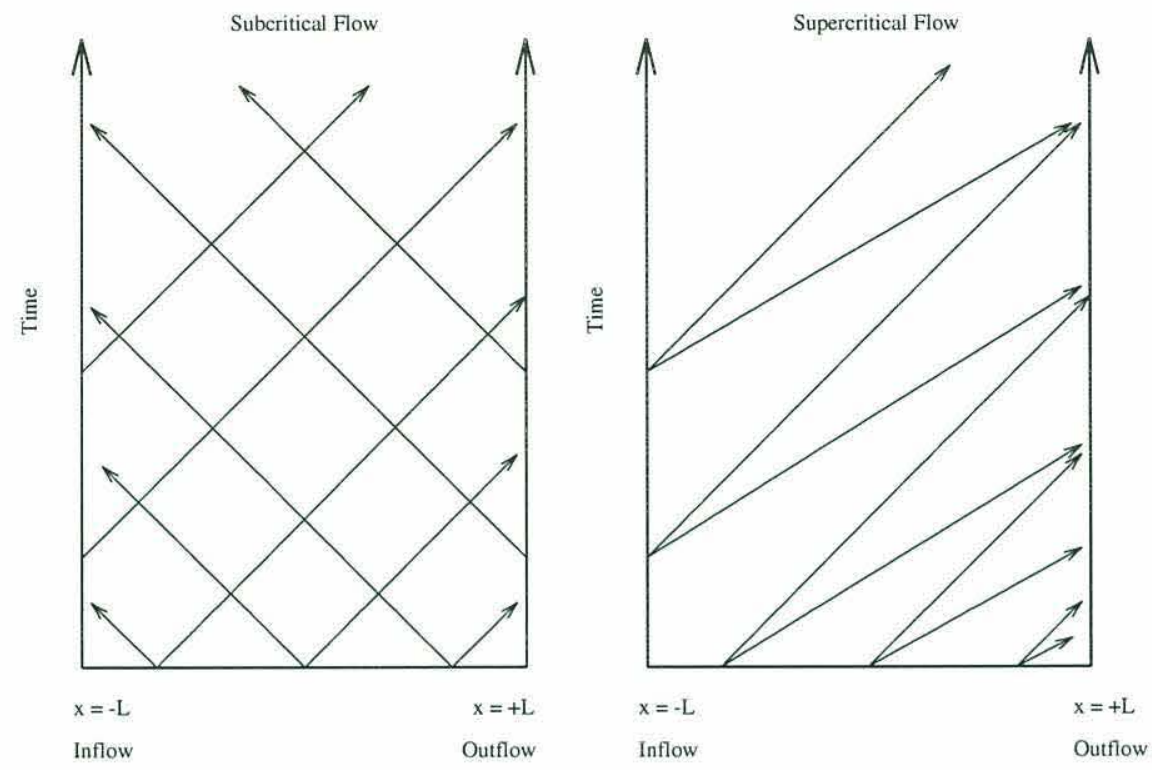

Figure 2.5: A schematic of the characteristics for supercritical and subcritical flow through a channel. The number of boundary conditions required at a given boundary to ensure a well posed solution is equal to the number of characteristics emanating from that boundary.

mined by the initial and upstream conditions. This situation is in marked contrast to the subcritical case, which has characteristics emanating from both boundaries, thus requiring that a single boundary value be supplied at the entrance and exit respectively. This discussion indicates that even for certain relatively simple systems, no general, pointwise open boundary condition can guarantee a mathematically well-posed problem.

Fortuitously, the supercritical condition, $U_{0}>C$, is not typical of oceanic flows. Thus, despite the many fundamental difficulties formulating rigorous mathematical open boundary conditions, it is possible to construct pragmatic conditions for numerical primitiveequation models. Because of the dispersive, nonlinear nature of the primitive-equations, derivation of exact or general open boundary conditions is not presently possible. Instead, a series of ad hoc numerical techniques have been developed using empirical testing on a case by case basis as the measure of success of the boundary condition.

Loosely based on the Sommerfeld radiation condition, the commonly used Orlanski boundary condition calculates a local propagation velocity, $C$, using information neighboring the boundary, rather than using a constant phase velocity [Orlanski 1975]. The 
Orlanski condition can be written:

$$
\frac{\partial \phi}{\partial t}+C_{\phi} \frac{\partial \phi}{\partial x}=0
$$

where $C_{\phi}$ is calculated using grid points adjacent to the boundary. The allowable magnitude of the calculated phase velocity is bounded above by the fastest wave speed supported given the grid size and timestep, and below by zero. Thus $C_{\phi}$ can be expressed as:

$$
C_{\phi}= \begin{cases}\frac{\Delta x}{\Delta t} & \text { if }-\frac{\partial \phi}{\partial t} / \frac{\partial \phi}{\partial x}>\frac{\Delta x}{\Delta t} \\ -\frac{\partial \phi}{\partial t} / \frac{\partial \phi}{\partial x} & \text { if } 0<-\frac{\partial \phi}{\partial t} / \frac{\partial \phi}{\partial x}<\frac{\Delta x}{\Delta t} \\ 0 & \text { if }-\frac{\partial \phi}{\partial t} / \frac{\partial \phi}{\partial x}<0\end{cases}
$$

Note that for each model variable, $\phi$, a separate phase velocity, $C_{\phi}$, can be calculated.

Because the Orlanski condition, applied to the three-dimensional dispersive wave problem, is an approximation, it is, not surprisingly, imperfect. In many cases the open boundary condition may imperfectly absorb energy or even generate anomalies which propagate back into the interior, thus having an effect on the solution. In order to minimize reflection and generation of energy it is common to combine the Orlanski condition with a viscous damping layer, or "sponge" layer. This combined boundary condition was introduced and discussed in some detail by Israeli and Orzag [1980]. An excellent comparative study of open boundary conditions, including both explicit and implicit numerical forms of the Orlanski condition, both with and without an associated viscous sponge layer, is presented in the context of a barotropic coastal ocean model by Chapman [1985].

\subsubsection{Specifics}

In configuring the open boundary condition for my own runs I have drawn directly on the code written by Chapman [1985]. The implicit numerical form of the Orlanski radiation condition at a boundary, $x=L$, is: 


$$
\phi_{L}^{n+1}=\frac{\phi_{L}^{n-1}(1-\mu)+2 \mu \phi_{L-1}^{n}}{1+\mu}
$$

where,

$$
\mu= \begin{cases}1 & \text { if } C \geq 1 \\ C & \text { if } 0<C<1 \\ 0 & \text { if } C \leq 0\end{cases}
$$

and,

$$
C=\frac{\phi_{L-1}^{n-1}-\phi_{L-1}^{n+1}}{\phi_{L-1}^{n+1}+\phi_{L-1}^{n-1}-2 \phi_{L-2}^{n}}
$$

I employ this numerical condition on the vertically averaged vorticity as well as on the vertically varying components of the horizontal velocity fields. The condition for temperature is calculated differently depending on the sign of the total velocity at the exit. For outflow portions of the boundary, I employ a zero gradient condition, which is equivalent to choosing a phase velocity for temperature of $C=\frac{\Delta x}{\Delta t}$. If, on the other hand, the velocity calculated at the open boundary is into the domain, I employ a fixed boundary condition, such that $C=0$. Numerically this condition is:

$$
\phi_{L}^{n+1}= \begin{cases}\phi_{L-1}^{n+1} & \text { if } u>0 \\ \phi_{L}^{n} & \text { if } u \leq 0\end{cases}
$$

As discussed in 2.3.1, an Orlanski radiation condition is not expected to perfectly absorb all incoming energy. In this case, the imperfection appears as an internal Kelvin wave, generated in the upper corner of the exit region, which propagates along the side wall, eventually fouling the interior solution. In order to remove this Kelvin wave it is necessary to incorporate a sponge layer near the exit.

After some experimenting with various configurations I chose to apply the sponge over the five grid cells nearest to the open boundary. The sponge consists of a linearly 
increasing Rayleigh damping term multiplying the depth dependant velocity fields and the barotropic vorticity field. Although I initially included a sponge layer for the temperature field as well, experimentation showed that it was not crucial for damping of the Kelvin wave. The magnitude of the frictional damping coefficient increases linearly from zero in the interior to 0.2 at the exit. Figure 2.6 shows a Taylor cap spinup run without this sponge. The Kelvin wave is visible as a density anomaly propagating into the domain from the upper corner of the exit. For comparison, recall that figure 2.3, which shows a spinup run with identical model settings but including a sponge layer, shows no evidence of Kelvin wave generation at the exit. Figure 2.7 shows a similar run in a longer channel using periodic boundary conditions. The excellent agreement between figures 2.7 and 2.3 demonstrates that the radiation condition and sponge layer have a negligible influence on the interior solution.

In conclusion, it seems that this boundary condition is adequate for the modeling I am doing. It allows anomalies and mean flow to leave the domain with minimal effect on the interior solution. However, the specific nature of the boundary condition is not supported by much of a theoretical base. Other than improving the condition empirically, by tuning the sponge parameters for example, there is no obvious method for improving the general applicability of the numerical condition. This particular condition, although robust within the parameter space I am running in, would, for example, probably fail if the mean flow were increased enough to move the flow into a supercritical regime. Unfortunately, it appears that each numerical model, and each model parameter configuration, must rely on empirical testing on a case by case basis to ensure the reliablility of any specific open boundary formulation.

\subsection{Surface Buoyancy Flux}

In its standard configuration the SPEM treats surface and bottom fluxes by direct specification of surface fluxes. There are, however, some inherent difficulties with the use of 

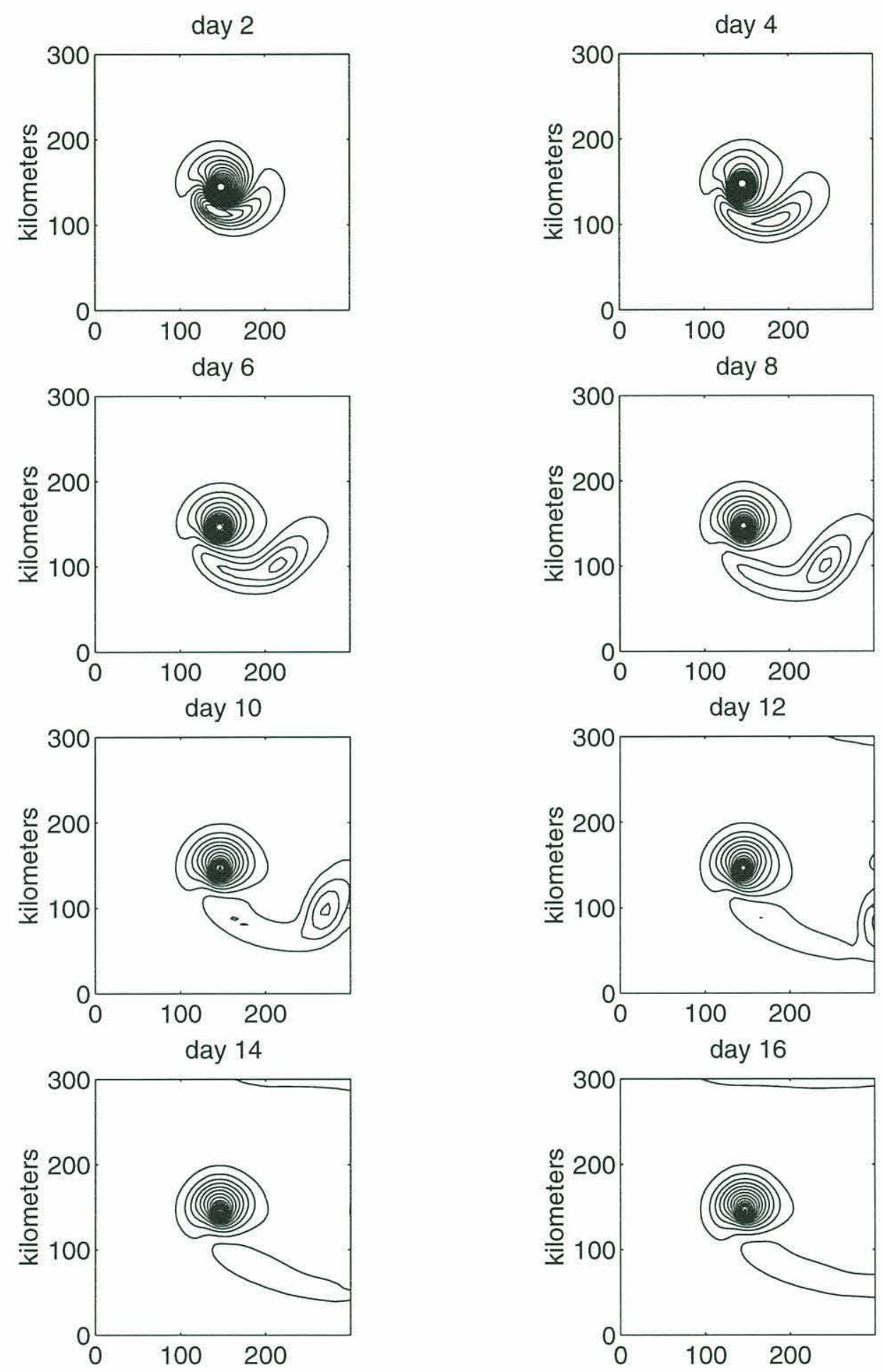

Figure 2.6: Temperature contours at 2000 meters for a Taylor cap spinup with an Orlanski open boundary condition. Although the cyclonic anomaly exits properly, a baroclinic Kelvin wave is generated in the upper corner of the exit region around day 12 . 
day 2
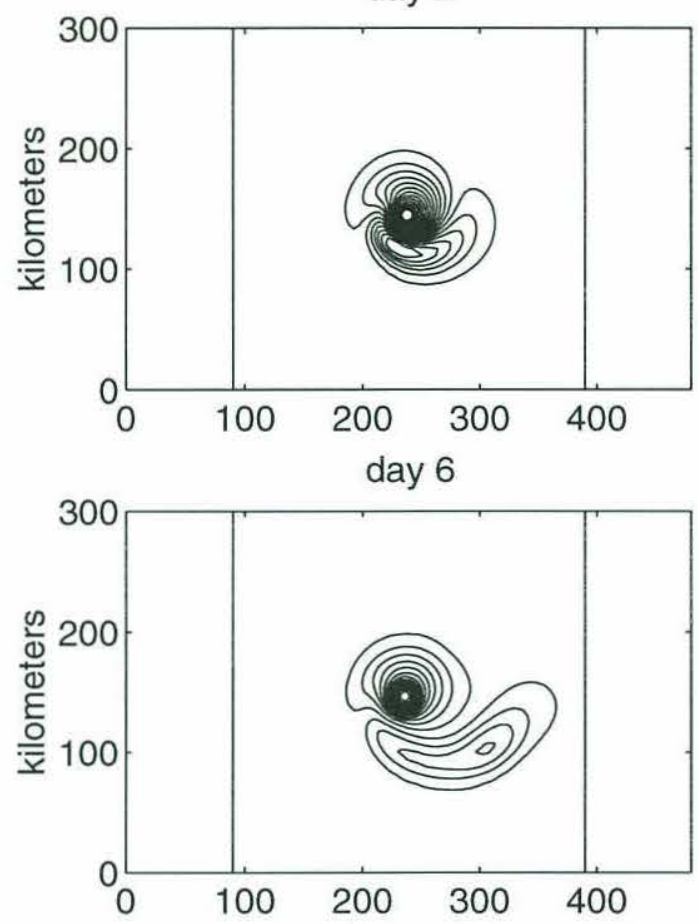

day 10
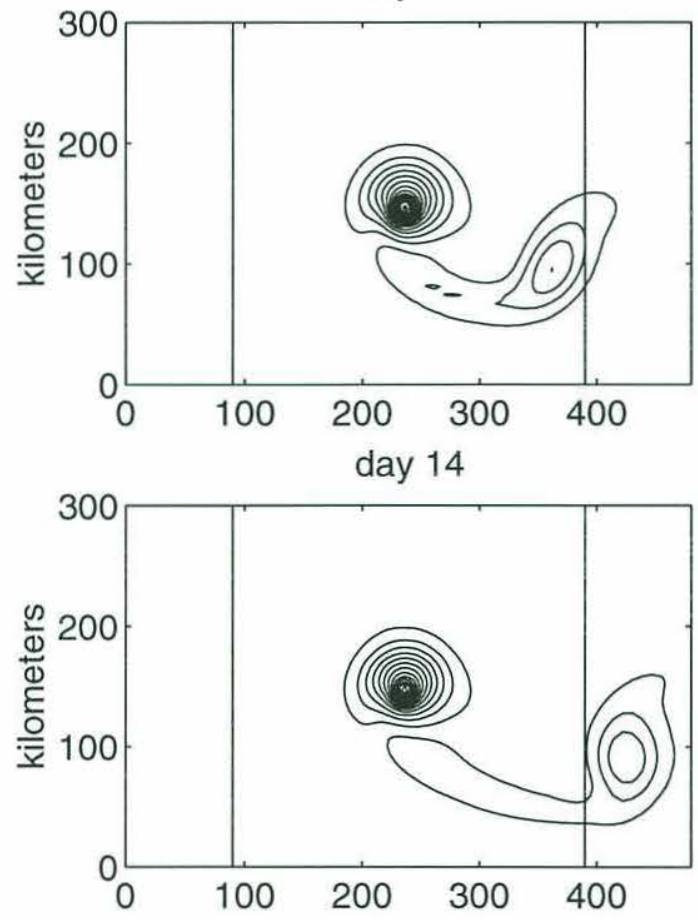

day 4
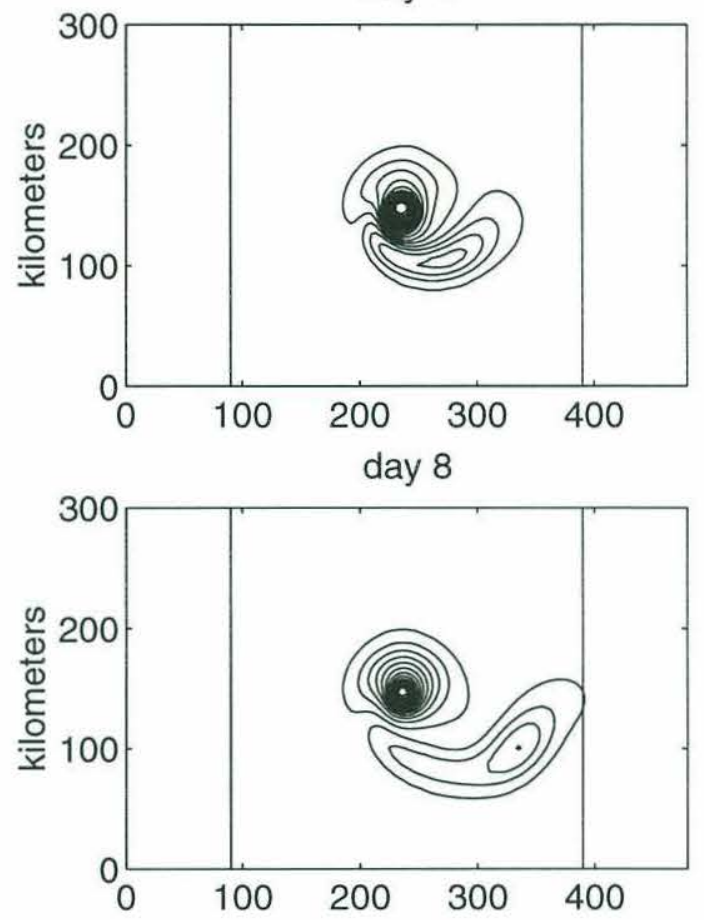

day 12
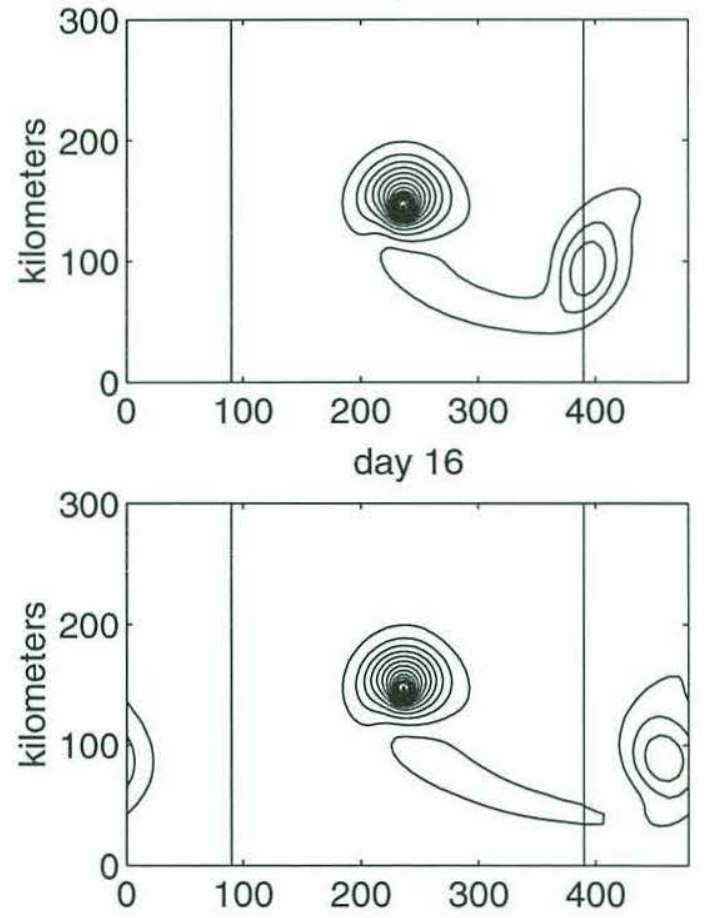

Figure 2.7: Temperature contours at 2000 meters for a Taylor cap spinup in a long domain with periodic boundary conditions. The solid vertical lines demarcate the smaller domain size for comparison with runs using open boundary conditions. 
this formulation when using a spectral vertical representation. Essentially, the surface flux appears as a delta function at the ocean surface in the vertical heat flux term in the heat conservation equation. The inability of a finite series expansion to represent such a singularity is the well known Gibbs effect. In practice, this difficulty produces spurious temperature signals in the deep water caused when surface cooling excites the highest order polynomial mode.

The vertical diffusion term for temperature, can be written:

$$
\frac{\partial}{\partial z}\left(\kappa \frac{\partial T}{\partial z}\right)
$$

In this form, the diffusion term can be thought of as the vertical derivative of the vertical temperature flux. The vertical temperature flux is the flux of heat in ${ }^{\circ} \mathrm{Cm} / \mathrm{s}$ between any two levels in the model. In the interior, this flux is simply given by $\kappa_{T} \frac{\partial T}{\partial z}$. This interior flux, in a given run, can be estimated using $\kappa=10^{-4} \mathrm{~m}^{2} / \mathrm{s}$ and $\frac{\partial T}{\partial z}=10^{-5 \circ} \mathrm{C} / \mathrm{m}$ to be of the order of $10^{-9 \circ} \mathrm{Cm} / \mathrm{s}$.

At the surface boundary the temperature flux is prescribed as a boundary condition proportional to the desired magnitude of surface cooling. The conversion factor between the temperature flux, $T$, as it is incorporated as an upper boundary condition in the SPEM and the more familiar heat flux, $H$, in $W m^{-2}$, and the buoyancy flux, $B$, in $m^{2} s^{-3}$ is:

$$
H=T C_{w} \rho=\frac{\rho C_{w} B}{g \alpha} .
$$

where $\alpha$ is the thermal expansion coefficient in ${ }^{\circ} C^{-1}$ and $C_{w}$ is the specific heat of seawater in Joules $/ \mathrm{Kg}^{\circ} \mathrm{C}$. A surface cooling of $40 \mathrm{wm}^{-2}$ thus corresponds to a surface temperature flux of $10^{-50} \mathrm{Cm} / \mathrm{s}$, which is 4 orders of magnitude greater than typical interior values, estimated above to be $10^{-9 \circ} \mathrm{Cm} / \mathrm{s}$. This huge vertical variation between the surface value and those in the interior must be differentiated in the vertical as per equation 2.21 in order to represent the surface flux in the temperature conservation equation. The subsequent excitation of the highest polynomial mode due to the inability 
of the finite spectral representation to capture such a large jump in temperature flux is demonstrated in figure 2.8 .

The upper panel of figure 2.8 shows the magnitude of the vertical diffusion term in the temperature conservation equation as a function of depth, with a surface heat flux equal to $40 \mathrm{w} / \mathrm{m}^{2}$. The excitation of the highest polynomial mode, whose structure is shown in dashed lines for comparison, is evident. The lower panel shows the magnitude of the same vertical diffusion term in the absence of surface forcing. Without surface forcing, there is still some Gibbs phenomenon error introduced due to the finite number of modes being used. However, the error is decreased by about eight orders of magnitude, to a very tolerable level.

The temperature anomalies produced in the deep water due to this excitation of the highest order mode are unfortunately quite robust. Consequently, I have modified the method by which surface buoyancy forcing is incorporated into the SPEM. One possible solution would be to simply remove the contribution of the highest order polynomial to the temperature field after each timestep. This is not entirely satisfactory however, not only because of the obvious sacrifice of vertical resolution, but also because any real information contained in this mode is lost. A more satisfactory solution is to incorporate the surface cooling using a simple slab mixed-layer formulation.

The mixed-layer which I have incorporated into the SPEM is in no way an attempt to accurately model mixed-layer dynamics. It merely serves as a simple mechanism for directly incorporating surface heat fluxes into the upper water column without exciting artificial deep temperature anomalies. The essential idea is to arbitrarily set a mixedlayer depth, which I have chosen to be one hundred meters, and evenly distribute the surface forcing within this layer. Since I am not using temporally or spatially varying, realistic surface fluxes, and am not particularly interested in the short term transient convective response to surface cooling, the lack of a dynamic mixed-layer is probably not of great importance. In addition, the depth of convective penetration is always deeper than the prescribed hundred meter mixed-layer depth. Thus, the interior model solutions 

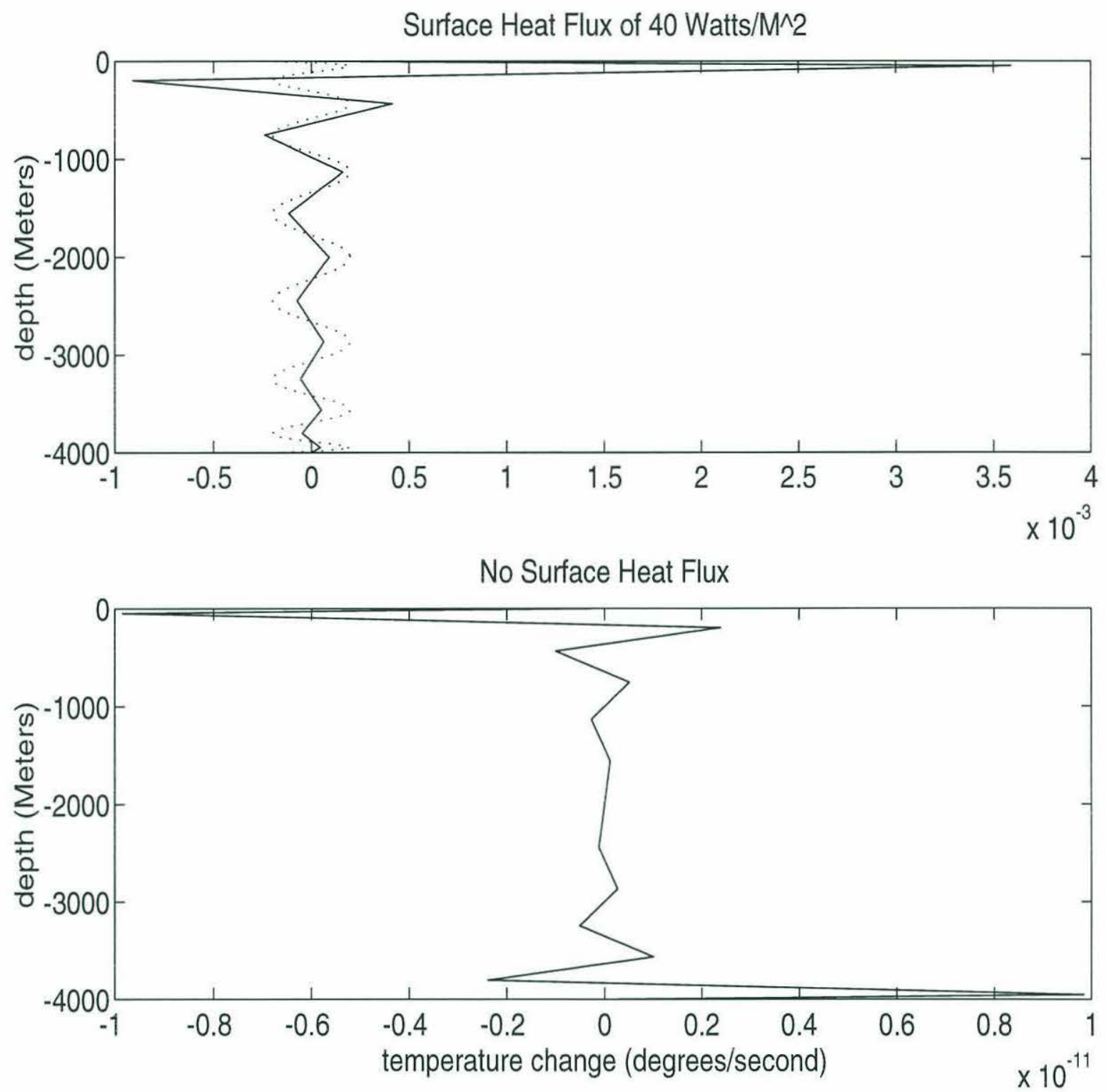

Figure 2.8: The upper panel shows the magnitude of the vertical diffusion term in the temperature conservation equation as a function of depth, with a surface heat flux proportional to $40 \mathrm{~W} / \mathrm{m}^{2}$. The lower panel shows the magnitude of the same vertical diffusion term in the absence of surface forcing. 
are robust, despite the oversimplification of surface processes. The specific details of the solution within the topmost hundred meters of the water column, however, should not, and indeed do not, play any role in interpretation of the model results.

Because the SPEM uses a sigma coordinate stretching and spectral representation of the vertical coordinate, the method of incorporating fluxes into the surface slab mixedlayer is not completely straightforward. First, it is determined which collocation points, located at the maxima of the highest spectral mode, are within the prescribed mixedlayer. Because of the large variations in bottom topography in many of my runs, the number of collocation points located within the surface mixed-layer is necessarily a function of horizontal spatial location. The surface flux per unit area is multiplied by the local surface area, which also varies horizontally due to the stretched grid, giving the total heat flux into the mixed-layer. This flux is then used to change the the temperature at the collocation points, weighted such as to ensure uniform cooling throughout the slab mixed-layer. If the mixed-layer density becomes greater than that of the underlying water column, additional deepening is ensured by the simple convective adjustment scheme used by the SPEM, which homogenizes the temperature vertically until static stability is achieved.

The utility of this slab mixed-layer formulation as a method for incorporating surface fluxes is most notable in the close match between the convective penetration depth measured from model output and analytic estimates of one-dimensional, non-penetrative convection. The model solutions also show little dependence on the exact value of the chosen mixed-layer depth. Finally, two experiments were performed to compare the mixed-layer formulation with a straightforward application of the standard flux condition provided with the SPEM. The model domain and grid for these runs was identical to that described in this chapter for flow over topography, except that the seamount has been removed and the surface forcing is applied in a 20 kilometer disk over the surface of an initially quiescent stratified fluid rather than being applied uniformly over a steady-state, Taylor cap flow. 
In both runs, as expected, a convective chimney forms below the cooling region. Figures 2.9 and 2.10 show time series of density slices of through the center of the chimneys over four days of cooling at $120 \mathrm{~W} / \mathrm{m}^{2}$. Figure 2.9, taken from the run made using the standard surface flux formulation, shows large spurious anomalies in the deep water well below the depth to which the convection has penetrated. When the surface mixed-layer formulation is used however, as shown in figure 2.10 , the deep anomalies are no longer present. Thus, this method of incorporating surface fluxes into a constant depth mixed-layer effectively solves the problem of excitation of the highest polynomial mode due to surface cooling. 

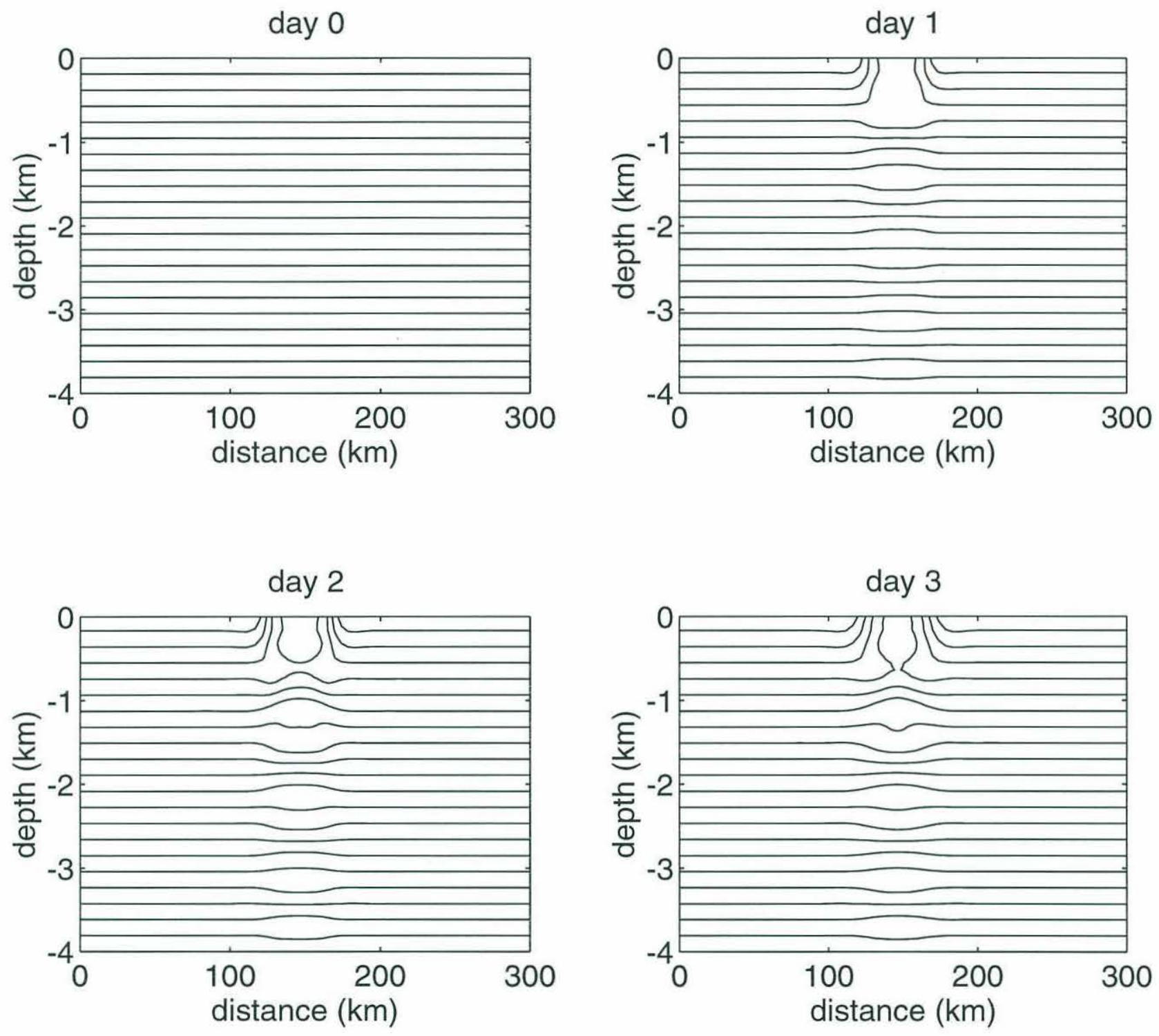

Figure 2.9: A slice through the center of the domain for a run with $120 \mathrm{~W} / \mathrm{m}^{2}$ cooling in a circular patch of radius 20 kilometers, using the surface flux representation of surface cooling provided with SPEM version 3.2. Note the excitation of the highest polynomial mode which shows up as large, spurious, displacements in the deep isopycnal structure, well below the depth of convective penetration. 

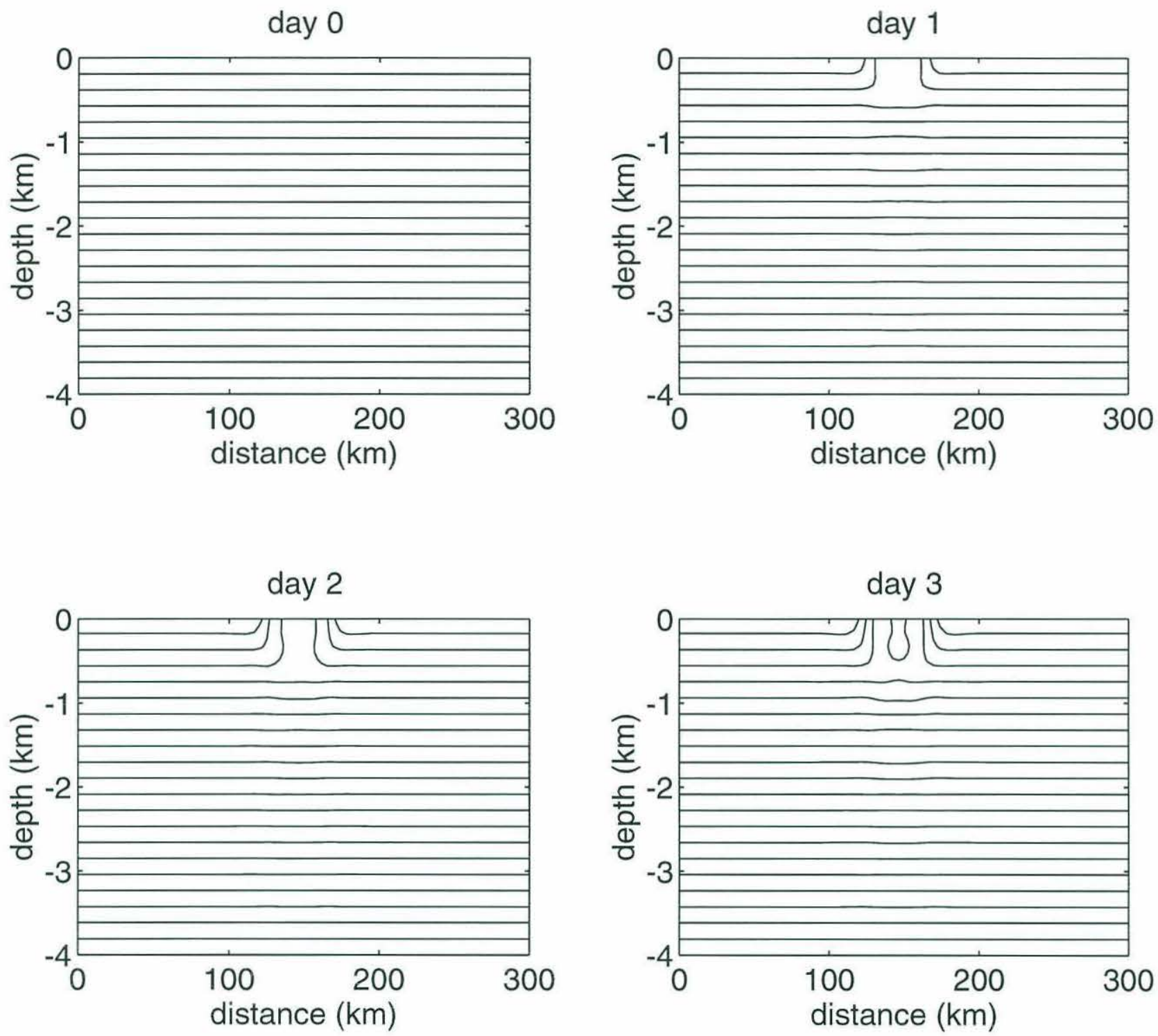

Figure 2.10: A slice through the center of the domain for a run with $120 \mathrm{~W} / \mathrm{m}^{2}$ cooling in a circular patch of radius 20 kilometers, using a surface mixed-layer formulation to incorporate the cooling. This formulation of the surface cooling effectively removes the problem of excitation of the highest polynomial mode. 


\section{Chapter 3}

\section{Topographic Preconditioning of Open Ocean Convection}

\subsection{Introduction}

There are at least two means by which mean flow topographic interaction can precondition the water column for convection: isolation of fluid over the seamount in a Taylor cap and doming of isopycnals associated with flow over and around the seamount. Domed isopycnals, which turn out to be of little importance when the background stratification is uniform, are examined in the context of surface intensified background stratification in Chapter 4. In this chapter, the trapping effect is examined.

\subsubsection{One-Dimensional Analytic Limit}

Before discussing the particulars of the numerical experiments it is worth considering the well known analytical limit for non-penetrative convection into an initially uniformly stratified ocean. Non-penetrative convection is defined simply as convection which penetrates only as far into the water column as the surface buoyancy flux allows, without additional deepening due to mechanical mixing or other effects. The one-dimensional 


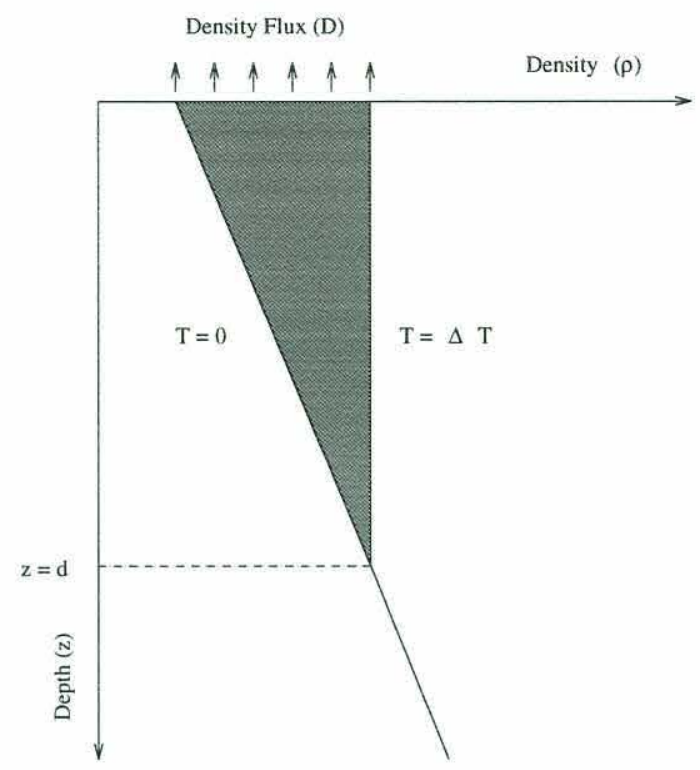

Figure 3.1: A schematic of one-dimensional, non-penetrative convection into a linearly stratified ocean. The shaded area represents the loss of buoyancy in a time $\Delta T$, which is equal to the total surface byouancy loss over that time. The convective penetration depth is $d$.

analytic convective depth serves as a useful comparison for many of the numerical results presented in this chapter.

Consider one-dimensional, non-penetrative convection into a constant background stratification as shown in figure 3.1. The depth of convection, $d$, is easily calculated by setting the time integrated surface buoyancy flux equal to the loss of buoyancy associated with the deepening of a surface well mixed-layer.

$$
B T=\frac{g}{\rho_{0}} \int_{-d}^{0}\left(\left.\rho(z)\right|_{t=T}-\left.\rho(z)\right|_{t=0}\right) d z
$$

Here, $B$ is the surface buoyancy loss, which remains constant in time, and the background linear stratification is given by $\left.\rho(z)\right|_{t=0}=-\frac{\rho_{0} N^{2} z}{g}$, where $N$ is the buoyancy frequency, which is assumed constant for simplicity. Assuming one-dimensional, non-penetrative convection, the temperature of the well mixed-layer after time $T$ is, by definition, a constant with the value $\left.\rho(z)\right|_{t=T}=\frac{\rho_{0} N^{2} d}{g}$. Substitution of these two expressions into equation 3.1 gives: 


$$
B T=\frac{g}{\rho_{0}} \int_{-d}^{0} \frac{\rho_{0} N^{2}}{g}(d+z) d z .
$$

Finally, solving for the convective depth leads to:

$$
d=\sqrt{\frac{2 B T}{N^{2}}}
$$

Equation 3.3 is the well known one-dimensional limit for non-penetrative convection into a linearly stratified fluid. This simple calculation assumes that as a convective chimney deepens into an initially stratified fluid, the negative surface heat flux is entirely balanced by cooling of surface water. In the simple framework of one-dimensional, non-penetrative convection, this surface cooling leads to further convective deepening as denser surface water mixes with the underlying fluid until hydrostatic stability is restored. Because the problem is one-dimensional, the only way to balance surface heat flux is through local cooling of the upper water column. However, when horizontal variability is allowed, a second mechanism for balancing surface heat loss comes into play, namely the lateral advection of heat.

\subsubsection{Local Trapping as Topographic Preconditioning}

Lateral fluxes of heat into a convecting chimney can be accomplished by mixing with the warmer stratified fluid surrounding the convecting patch. This mixing can be accomplished by eddy fluxes or by mean flow. Note that it is highly unlikely that denser water will be available to advect laterally into a deep convecting chimney. Consequently, the effect of lateral mixing will always be to reduce the convective penetration depth.

Isolation of a region of fluid in a Taylor cap over bottom topography within a larger region of significant mean flow can ensure that lateral fluxes of heat are minimized. Consequently, all surface buoyancy fluxes over the region of trapped flow must be balanced by local cooling, allowing the convective depth to approach the one-dimensional limit given by equation 3.3. Away from the topographic trapping however, horizontal fluxes 
of heat by the mean flow will tend to shut down the mixed-layer deepening at a shallower depth than the one-dimensional calculation suggests. Thus, an isolated Taylor cap may serve as a pre-existing conduit into the deep water through which ventilation can occur.

\subsection{Surface Mixed-Layer Depth}

Consider first the surface mixed-layer in isolation, neglecting for the moment the presence of topography and any associated deflection of the mean flow. At the time that cooling is first initiated, a mixed-layer will begin to deepen uniformly throughout the domain. Near the inflow, however, this mixed-layer will immediately be influenced by advection of stratified water into the domain. Soon, the depth of convection will reach a steady-state maximum with the surface negative buoyancy flux entirely balanced by the influx of more buoyant water laterally. The further away from the inflow one gets, the longer it will take for high buoyancy stratified water to be advected in and shut down the convective deepening.

From a Lagrangian point of view, a particle entering at the surface travels at a speed $U$ to a point a distance $x$ from the inflow in a time $x / U$. Assuming that the particle never leaves the mixed-layer, it will feel the surface cooling for its entire passage through the domain. The mixed-layer depth as a function of downstream position can thus be estimated by substituting this advective timescale, $x / U$, into the non-penetrative, onedimensional estimate for convective depth given by equation 3.3. This substitution gives an analytical estimate for the steady-state, mixed-layer depth as a function of downstream distance from the inflow:

$$
d=\sqrt{\frac{2 B x}{U N^{2}}}
$$

Thus, while the absolute depth of the mixed-layer is dependent on the magnitudes of the buoyancy forcing, the mean flow velocity and the initial stratification, its shape is always going to be given by a square root dependence on distance downstream from the inflow. 
It is important to note that the mixed-layer depth is actually in a steady-state, representing the maximum possible convective penetration. Once this steady-state has been achieved, surface cooling is in exact balance with the influx of heat associated with the inflow of stratified water and does not result in further convection. The extent to which the results of these experiments are dependent on this particular mechanism for constraining ambient mixed layer depth is of interest. Clearly, were the domain to be periodic, and neglecting the effect of bottom topography, the mixed layer would simply deepen uniformly as long as cooling was applied. The only way, in that case, to compare ambient levels of cooling with those over the seamount would be to cool for a finite period of time. In the context of flow in the Weddell Gyre, mixed layer depth is set by a combination of many factors, including the integrated amount of cooling in a winter season.

However, it is also true that the "incoming" flow which runs over Maud Rise from the Northeast, is entering the region of most intense negative buoyancy forcing as it moves southward and that its initial stratification is set, to some degree, by the influx of intermediate waters of North Atlantic origin. Thus, the simple experimental setup which calls for initially stratified water to continually flow into the domain and all of the cooling to occur within this domain is not without an oceanographic analogue. Although the specific physics governing mixed layer depth in this model are clearly oversimplified, they are not unreasonable. In addition, the presence of a large scale ambient mixed layer depth throughout the computation domain does reproduce, to first order, the presence of a large scale seasonal mixed layer in the Weddell Sea. The details of how this mixed layer depth is set have been parameterized within as simple a model construction as possible. It is hoped that this simplification does not alter the basic physical interaction of the mixed layer with flow over topography upon which the conclusions depend.

Figure 3.2 shows the spinup of a steady-state, mixed-layer in run M1. For this run the mixed-layer behavior has been isolated by removing topography from the problem. The mean flow, $\mathrm{U}$, has a value of $20 \mathrm{~cm} / \mathrm{sec}$, the Brunt-Vaisala frequency, $\mathrm{N}$, is $5.556 \times 10^{-4} \mathrm{~s}^{-1}$ and the buoyancy flux, B, is $1.5 \times 10^{-7} \mathrm{~m}^{2} \mathrm{~s}^{-3}$. The mixed-layer depth diagnosed from this 
run is in excellent agreement with the predicted depth given by equation 3.4 , as shown in figure 3.3 .

When bottom topography is included, the path which a particle takes through the domain is not a straight zonal line and it is not traversed at a constant velocity. Away from the seamount's influence, the mixed-layer maintains its simple square root dependence on downstream distance. Along streamlines which approach the vicinity of the seamount, however, some of the flow is significantly retarded while traversing a deflected, more lengthy, route through the domain. For example, the surface velocity field for run S2 is shown in figure 3.4 together with contours of the magnitude of the zonal component of the velocity. Clearly, the flow on the right hand side of the topography, looking downstream, is significantly retarded. Thus, along those streamlines with reduced zonal velocities the mixed-layer depth increases relative to elsewhere in the domain due to the greater cooling felt by particles spending more time in the domain.

The extent of this effect can be quantified by integrating the local velocity along a streamline and using it to calculate the time spent in the domain, which can then be substituted into equation 3.3. Rather than attempting to track particles through the threedimensional domain, an approximation can be obtained by taking the two-dimensional surface velocity field and calculating the minimum zonal velocity as a function of downstream position. Summing local grid size divided by minimum zonal velocity from inflow to outflow gives an upper bound on the time a particle in the mixed-layer can spend in the domain. Regions of zero or reverse flow associated with fluid trapped in the Taylor cap are ignored for this calculation, which is primarily an attempt to examine the background mixed-layer depth, away from the region of fluid trapping. Figure 3.5 shows the calculated mixed-layer penetration depth derived using this procedure as compared to that for undeflected uniform flow. The major deviation between the two curves occurs, not surprisingly, near the center of the domain, around 150 kilometers downstream, where the surface flow is most significantly retarded.

For the purposes of parameter space investigation, it is convenient to nondimension- 

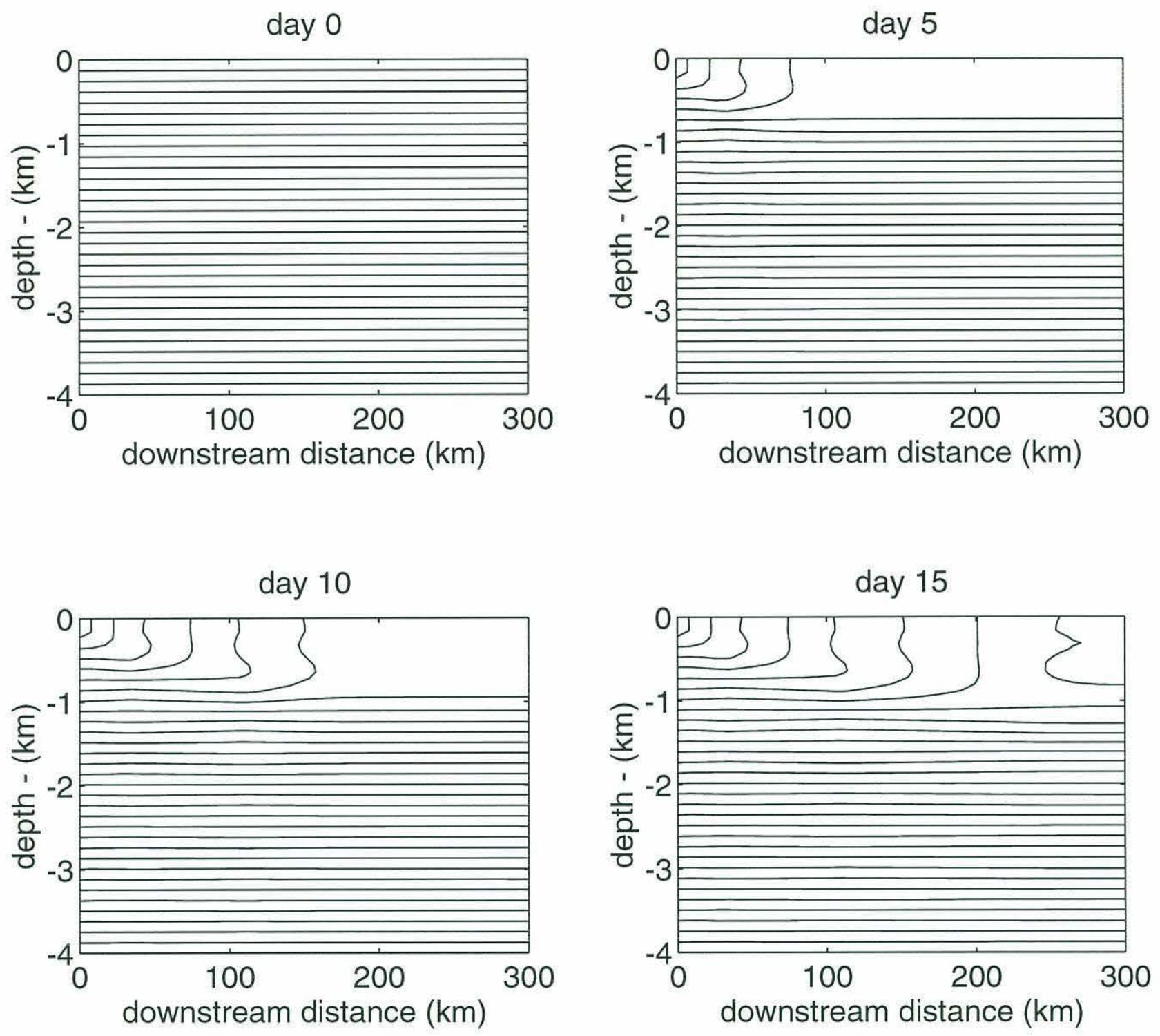

Figure 3.2: Evolution of a surface mixed-layer for run M1, with no bottom topography, initially uniform stratification, constant negative surface buoyancy forcing, and uniform mean flow. 


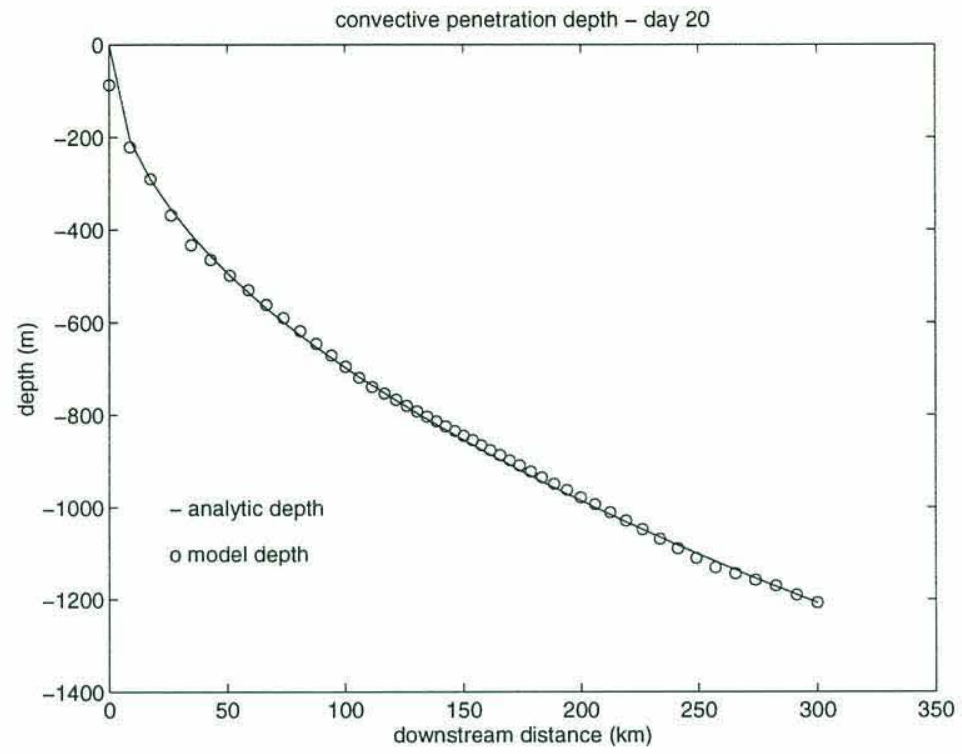

Figure 3.3: Steady-state mixed-layer depth as a function of distance downstream from the inflow as diagnosed from model fields at day 20 for run M1 (circles). The analytic solution from a Lagrangian one-dimensional, non-penetrative calculation is also shown (solid line).

alize the surface forcing. Following the Jones and Marshall [1993] modeling study on convection into neutrally stratified fluid, I have chosen to use the convective Rossby Number to nondimensionalize the buoyancy forcing with respect to the rate of rotation, $f$, and the total fluid depth, $H$.

$$
R_{N}=\sqrt{\frac{B}{f^{3} H^{2}}}
$$

In the context of my experiments, the convective Rossby Number serves primarily as a convenient nondimensional representation of the buoyancy flux. However, in the unstratified context, the convective Rossby Number can be thought of as the fractional depth of penetration of rotationally controlled convection. Such a ratio can also be calculated for the mixed-layer penetration depth estimate given by equation 3.4 divided by the total fluid depth, $H$. From the definition of convective Rossby Number, equation, we know:

$$
B^{1 / 2}=R_{N}\left(f^{3} H^{2}\right)^{1 / 2}
$$




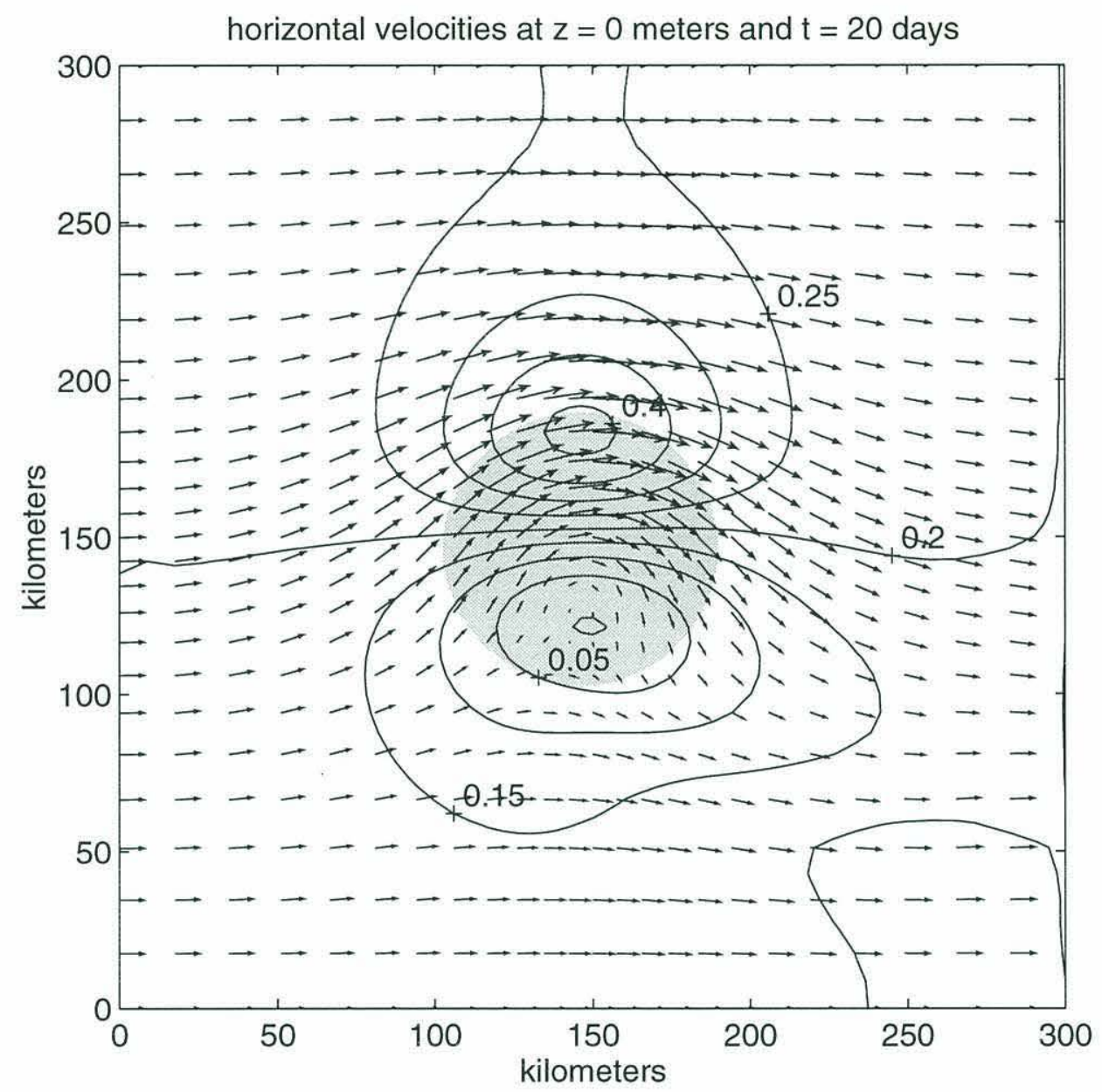

Figure 3.4: The steady-state horizontal velocity field at the surface for run S2. The contours show the magnitude of the zonal component of the total velocity, which is denoted by the arrow field. The region directly overlying the topography is shaded. 


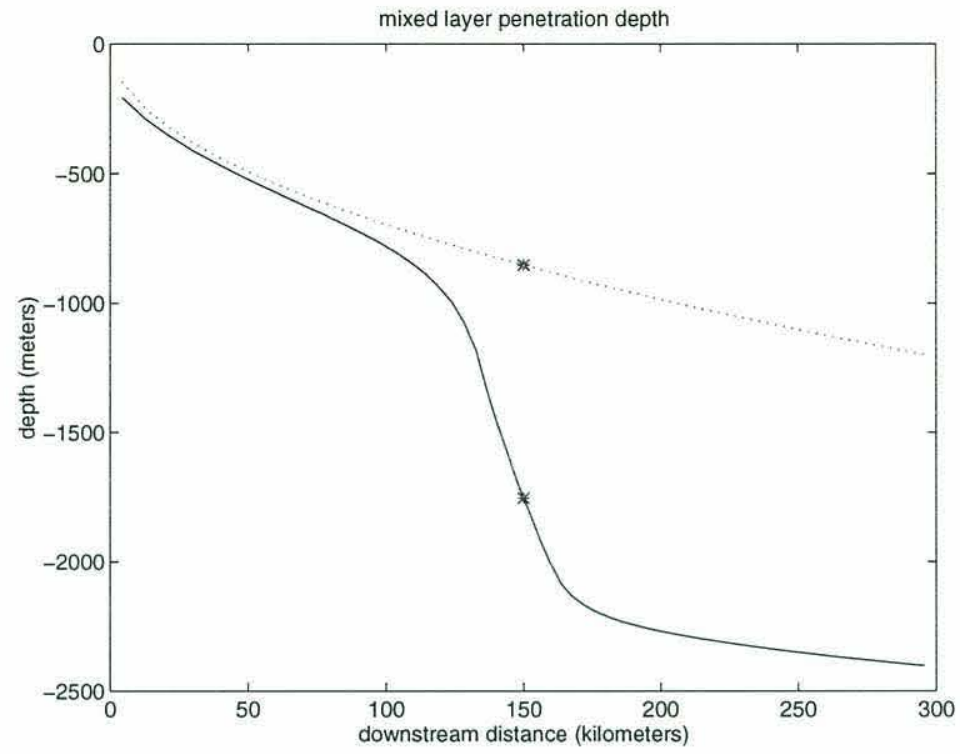

Figure 3.5: Steady-state mixed-layer depth as a function of distance downstream from the inflow for run S2. The dotted line shows the analytic solution in the constant velocity region away from the influence of bottom topography. The solid line shows an upper bound on the enhanced deepening in the vicinity of the seamount. The asterisks indicate the mixed-layer depth at 150 kilometers downstream, in the vicinity of the seamount.

Substitution into equation 3.4 leads to:

$$
\frac{d}{H}=R_{N}\left(\frac{2 f^{3} x}{U N^{2}}\right)^{1 / 2}=\sqrt{2} R_{N}\left(\frac{f}{N}\right)\left(\frac{f x}{U}\right)^{1 / 2}
$$

Finally, equation 3.7 can be simply rewritten in terms of the nondimensional parameters listed in Table 2.1 (the Rossby Number, R, and the stratification parameter, E) and the nondimensionalized downstream distance $\frac{x}{L}$ giving an expression for the ratio of expected convective depth to total depth for this problem:

$$
\frac{d}{H}=\frac{\sqrt{2} R_{N}}{E R^{1 / 2}}\left(\frac{x}{L}\right)^{\frac{1}{2}} .
$$

\subsection{Taylor Cap Height}

Consider now the effect of isolated bottom topography decoupled from the surface forcing. After an initial spinup, a steady-state flow over and around the topography can 
generally be found. As discussed earlier, the salient features of this flow important for their preconditioning effects are isopycnal doming over the seamount and the extent to which the flow is trapped over the seamount, isolated from horizontal advection. The next step, then, is to attempt to quantify to what distance above the seamount the region of trapped flow extends. In general, the expected height of penetration of topographic effects is $\mathrm{fL} / \mathrm{N}$. However, the height to which fluid trapping extends above the seamount is likely to be lower than this scale, which is more likely to approximate the height to which isopycnal doming extends into the water column. The actual Taylor cap height can be diagnosed, as a function of model parameters, directly from the model fields.

Because the surface buoyancy flux is not yet turned on when the Taylor cap is initially spun up, only four of the nondimensional parameters listed in table 2.1 are important in describing the Taylor cap: Rossby Number, stratification parameter, fractional height, and aspect ratio. An exhaustive mapping of parameter space in order to determine power law type dependencies for Taylor cap height on each of these four nondimensional parameters, although possible, would require an enormous number of model runs. Instead, I have limited the parameter space search to a few dozen runs in order to gain some insight into how each parameter effects Taylor cap height. An exact quantification of the dependence is not necessary in any case. Table 3.1 shows all of the Taylor cap spinup runs used to map out the parameter dependencies. In addition, Table 3.2 shows a subset of these runs which are referenced by name in the text.

Several diagnostics for determining the Taylor cap height from standard model fields can be developed. The two I have chosen to work with are the presence of a flow reversal in the horizontal velocity field and the distribution of a passive tracer. These diagnostics are discussed in sections 3.3 .1 and 3.3 .2 respectively.

\subsubsection{Flow Reversal Diagnostic}

One necessary condition for the flow to be trapped is the presence of a flow reversal in the velocity field on an isopycnal surface. Thus, searching for the distance above the 


\begin{tabular}{|l|l|l|l|l|l|l|l|l|}
\hline$N / f$ & $\delta=0.1$ & $\delta=0.2$ & $\delta=0.3$ & $\delta=0.4$ & $\delta=0.5$ & $\delta=0.6$ & $\delta=0.7$ & $\delta=0.8$ \\
\hline & & & & & & & & \\
1 & & $\mathrm{R}=0.08$ & & & $\mathrm{R}=0.08$ & & & \\
\hline 3 & & $\mathrm{R}=0.08$ & & & $\mathrm{R}=0.08$ & & & \\
\hline 4 & & & & & $\mathrm{R}=0.08$ & & & \\
\hline 5.556 & $\mathrm{R}=0.08$ & $\mathrm{R}=0.08$ & $\mathrm{R}=0.04$ & $\mathrm{R}=0.08$ & $\mathrm{R}=0.04$ & $\mathrm{R}=0.04$ & $\mathrm{R}=0.08$ & $\mathrm{R}=0.08$ \\
& & & $\mathrm{R}=0.06$ & & $\mathrm{R}=0.06$ & $\mathrm{R}=0.06$ & & \\
& & & $\mathrm{R}=0.08$ & & $\mathrm{R}=0.08$ & $\mathrm{R}=0.08$ & & \\
& & & $\mathrm{R}=0.10$ & & $\mathrm{R}=0.10$ & & & \\
\hline 7 & & & & & $\mathrm{R}=0.12$ & & & \\
\hline 8 & & & & & $\mathrm{R}=0.08$ & & & \\
\hline
\end{tabular}

Table 3.1: A listing of all the Taylor cap spinup runs used to map out the parameter dependencies of Taylor cap height. The three parameters varied are the fractional height, $\delta$, the stratifcation parameter, $N / f$, and the Rossby number, $R$. The seamount aspect ratio, $\Delta$, was held constant in all runs, with a value of 0.16 .

\begin{tabular}{|l|c|c|c|c|c|}
\hline Run & $\begin{array}{c}\text { Rossby } \\
\text { Number }(\mathrm{R})\end{array}$ & $\begin{array}{c}\text { Stratification } \\
\text { Parameter }(\mathrm{E})\end{array}$ & $\begin{array}{c}\text { Fractional } \\
\text { Height }(\delta)\end{array}$ & $\begin{array}{c}\text { Aspect Ratio } \\
(\Delta)\end{array}$ & $\begin{array}{c}\text { convective Rossby } \\
\text { Number } R_{N}\end{array}$ \\
\hline S1 & 0.08 & 3 & 0.05 & 0.16 & 0 \\
\hline S2 & 0.08 & 5.556 & 0.05 & 0.16 & 0 \\
\hline S3 & 0.08 & 7 & 0.05 & 0.16 & 0 \\
\hline M1 & 0.08 & 5.556 & 0 & 0 & $9.7 \times 10^{-2}$ \\
\hline
\end{tabular}

Table 3.2: Selected runs referred to in the text. 
seamount at which there is no longer a significant flow reversal in the steady-state velocity field is perhaps the simplest method for demarcating the upper limit of the Taylor cap. Rather than interpolating the model velocities onto isopycnal surfaces, it is convenient to search for flow reversals in the horizontal velocity fields themselves. The error introduced due to this simplification is small relative to the vertical resolution of the model in any case.

Figure 3.6 compares the minimum zonal velocities as a function of cross-stream distance for four runs, all with seamounts reaching to 2000 meters depth and mean flows of $20 \mathrm{~cm} / \mathrm{s}$, but with different $N / f$ values of $3.0,5.6,7$, and 8 respectively. Note that the run with the weakest stratification, $N / f=3$, is run $\mathrm{S} 1$ and the run with $N / f=5.556$ is run S2. Run S1, shown in the upper left hand panel, shows a pronounced flow reversal between about 120 and 180 kilometers cross-stream distance. There is little variability with height above the seamount, with flow reversals greater than $5 \mathrm{~cm} / \mathrm{s}$ at all depths, implying a nearly barotropic flow pattern with the Taylor cap reaching all the way to the surface. Were the domain deeper, it is likely that the Taylor cap would extend significantly higher than the 2000 meters associated with the upper surface in this run.

For run S2, shown in the upper right panel of figure 3.6, there is a greater vertical shear in the horizontal velocities. Just above the crest of the seamount, at 2000 meters depth, there is still a strong, $5 \mathrm{~cm} / \mathrm{s}$ flow reversal from about 130 to 170 kilometers crossstream distance, indicating the presence of a Taylor cap at that depth. At the surface, however, the flow reversal is extremely small (less than $1 \mathrm{~mm} / \mathrm{sec}$ ), and appears only at a single gridpoint. For this run, the Taylor cap seems to extend 2000 meters from the seamount peak, just barely to the surface. As the value of the stratification is further increased the Taylor cap height is further reduced. The lower left panel shows run S3, with $N / f=7$, which has a Taylor cap which reaches to 1500 meters depth, 500 meters above the seamount peak. In the lower right hand panel, a run with $N / f=8$ has a still lower Taylor cap height of only 200 meters. 

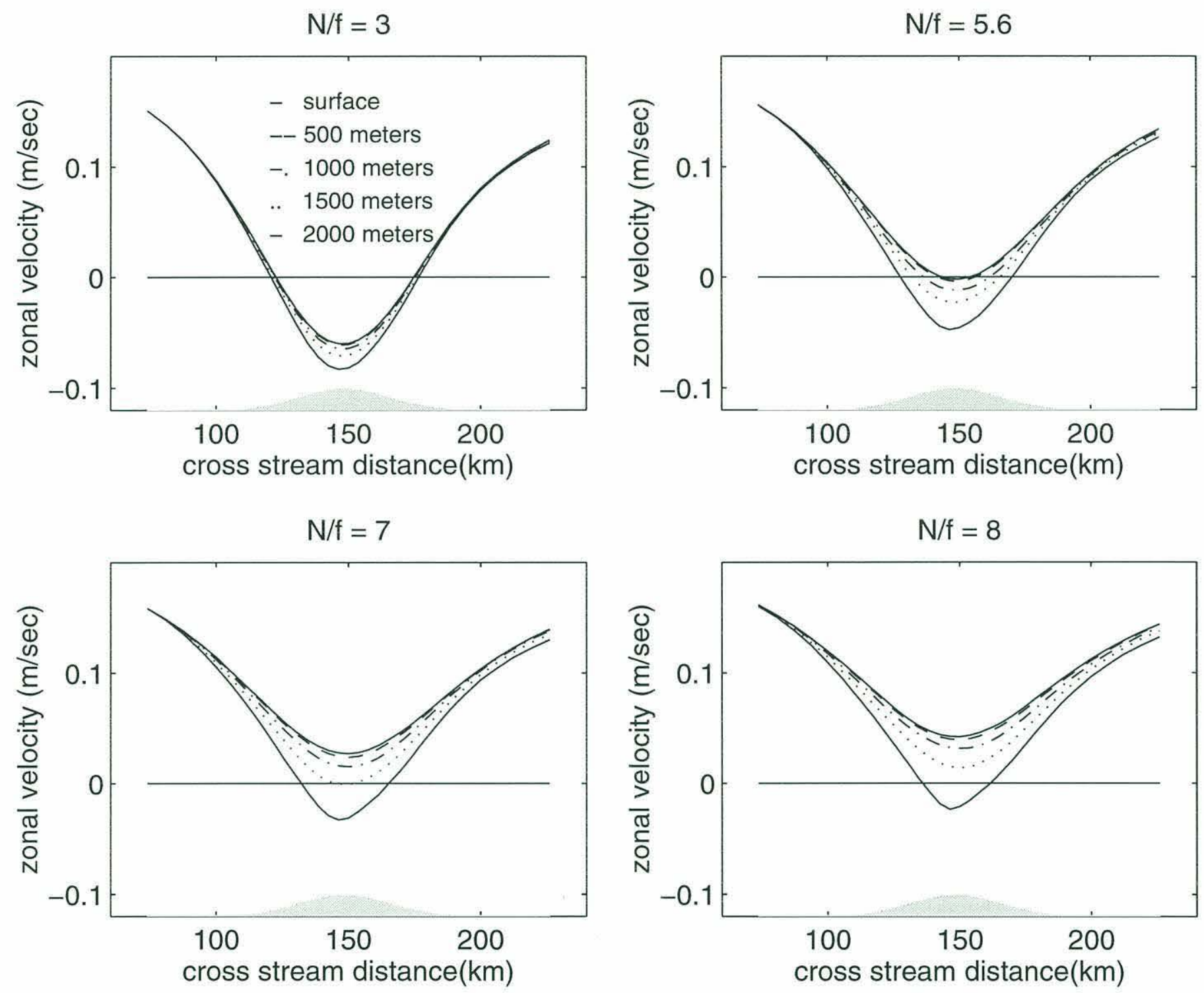

Figure 3.6: Minimum zonal velocities as a function of distance across the domain at various depths in four runs with different values of stratification parameter. The maximum height at which a flow reversal occurs indicates the height of the Taylor cap. 


\subsubsection{Passive Tracer Diagnostic}

Another method for diagnosing Taylor cap height is to seed the water column above the seamount with neutrally buoyant floats in order to map out the Taylor cap structure. Using this method, Chapman and Haidvogel [1992] conclude that increased inflow velocities reduce the Taylor cap height in a stratified flow. They do not attempt to quantify the parameter dependence beyond this qualitative statement however. The process of calculating float trajectories is not computationally negligible, meaning only a limited number of floats can reasonably be tracked. In addition, because of the finite Eulerian grid, some error is necessarily introduced in the approximation of Lagrangian particle trajectories, although this error may be small.

Instead of seeding with individual floats, it is also possible to spin up the Taylor cap with an initially uniform tracer value of zero. Once a steady-state has been achieved the inflow condition can be set to bring in tracer of value one. After the domain has been fully ventilated with this new tracer value from the inflow, the shape of the Taylor cap should be reflected in the distribution of the initial tracer value remaining in the domain. The tracer can be thought of as a continuum of neutrally buoyant floats. Unfortunately, such a passive advected tracer is not numerically stable. In particular, sharp gradients in the tracer distribution, which tend to develop over the seamount, are not well represented with the SPEM centered difference advection formulation. Consequently, as with the dynamical variables, a diffusion term is required to ensure numerical stability. The presence of the diffusion term means that the tracer field is no longer an exact representation of fluid particle trajectories. Thus, like the neutrally buoyant floats, the tracer field approximately delineates, but is not an exact measure of, the trapped region.

In the SPEM model the tracer equation is:

$$
\begin{aligned}
\frac{\partial S}{\partial t}+\vec{u} \cdot \nabla S & =F_{S} \\
F_{S} & =\kappa_{S} \nabla^{4} S
\end{aligned}
$$

where $\mathrm{S}$ denotes the the tracer concentration and $F_{S}$ is a biharmonic diffusion term acting 
along sigma surfaces with a value analogous to the diffusion terms in the momentum and temperature equations. $\kappa_{S}$ has a value of $4 \times 10^{9}$, identical to the corresponding value for temperature.

Figure 3.7 shows slices through the center of the domain for runs S2 and S3. In each run the steady state taylor cap has been spun up with an initally uniform tracer value of zero and as well as an inflow value of zero. The inflow tracer value is then increased gradually from zero to one over the two days and is then left at one for another 20 days. The initial gradual increase is necessary in order to avoid too strong a gradient in the tracer field, which leads to numerical instabilities. In the steady-state, any region which contains a tracer value of zero must be made up of water which is left over from the initial state, unaffected by the inflow. In both runs, a Taylor cap is visible as a region of zero tracer over the right flank of the seamount looking downstream. The tracer diagnostic suggests that the Taylor cap extends to the surface in the lower stratification run, S2, but reaches only to a depth of about 1500 meters with the stronger background stratification in run $\mathrm{S} 3$.

As a consequence of the small velocities to the left of the Taylor cap looking upstream, diffusion plays a stronger role in this area and smears out the tracer field somewhat. In figure 3.7 the zero contour is shown along with the 0.5 and 0.9 contours. Values of the tracer which are between zero and 1 are either due to the diffusion term in the tracer advection equation or from the initial two day long increase in tracer value from 0 to 1. The large region covered by these intermediate tracer values gives some idea of the uncertainty in this measurement, which is unfortunately at least several hundred meters in the vertical and tens of kilometers in the horizontal. Nevertheless, using the zero contour as an indicator of Taylor cap height suggests that, for run S2, there is a roughly conical shaped Taylor cap which reaches to the surface. In contrast, in the run S3, with stronger stratification, the zero contour maps out a Taylor cap extending to 1500 meters depth, only 500 meters above the seamount peak. These values for Taylor cap height are consistant with the values diagnosed using the flow reversal diagnostic. 


\subsubsection{Taylor Cap Height Parameter Dependencies}

The diagnostics discussed above allow a quantitative assessment of the height above the seamount peak to which the Taylor cap extends, referred to hereafter as Taylor cap height, and its dependence on the four nondimensional parameters. The diagnostics are broadly consistent with each other. However, the tracer diagnostic is not used because it does not give a very tight constraint on height. Thus, for the following discussion on parameter dependencies of Taylor cap height, the maximum height at which a flow reversal is found in the horizontal velocity field is used to infer the values for Taylor cap height in the various runs. An error bar of \pm 150 meters, given by the midepth vertical reslolution of the model, is assigned assigned to this value. In a few cases there is a flow reversal on the flank of the seamount, indicating a region of trapped flow, but there is no flow reversal at a level higher than the seamount peak itself. In these cases, the Taylor cap height, as defined as the distance above the seamount peak, is zero, although there is some topographic trapping of fluid.

Figure 3.8 shows Taylor cap height as a function of Rossby Number for two different seamount fractional heights. For the taller seamount, with a fractional height of 0.5 , there is a fairly tight range of Rossby Number over which the height varies. The critical Rossby Number, above which no recirculation exists over the topography, is between 0.1 and 0.2 , while for Rossby Numbers of 0.08 or lower, the recirculation extends to the surface. If the Taylor cap is diagnosed as hitting the upper surface, denoted as the dotted line, then this height is of course not the same as one would get in an arbitrarily deep domain. For the shorter seamount, the critical Rossby Number is also around 0.1. In addition, only the lowest Rossby Number, 0.04, allows the trapped region to extend to the surface. For the lower seamount the range of Rossby numbers which support Taylor cap's of intermediate depth is somewhat wider than for the taller seamount.

The dependence on $N / f$ is shown in figure 3.9, with Rossby number and fractional height held constant at values of 0.08 and 0.5 respectively. For $N / f$ of 5.6 or lower, the Taylor cap reaches the surface. The height of the cap decreases rapidly with $N / f$. The 

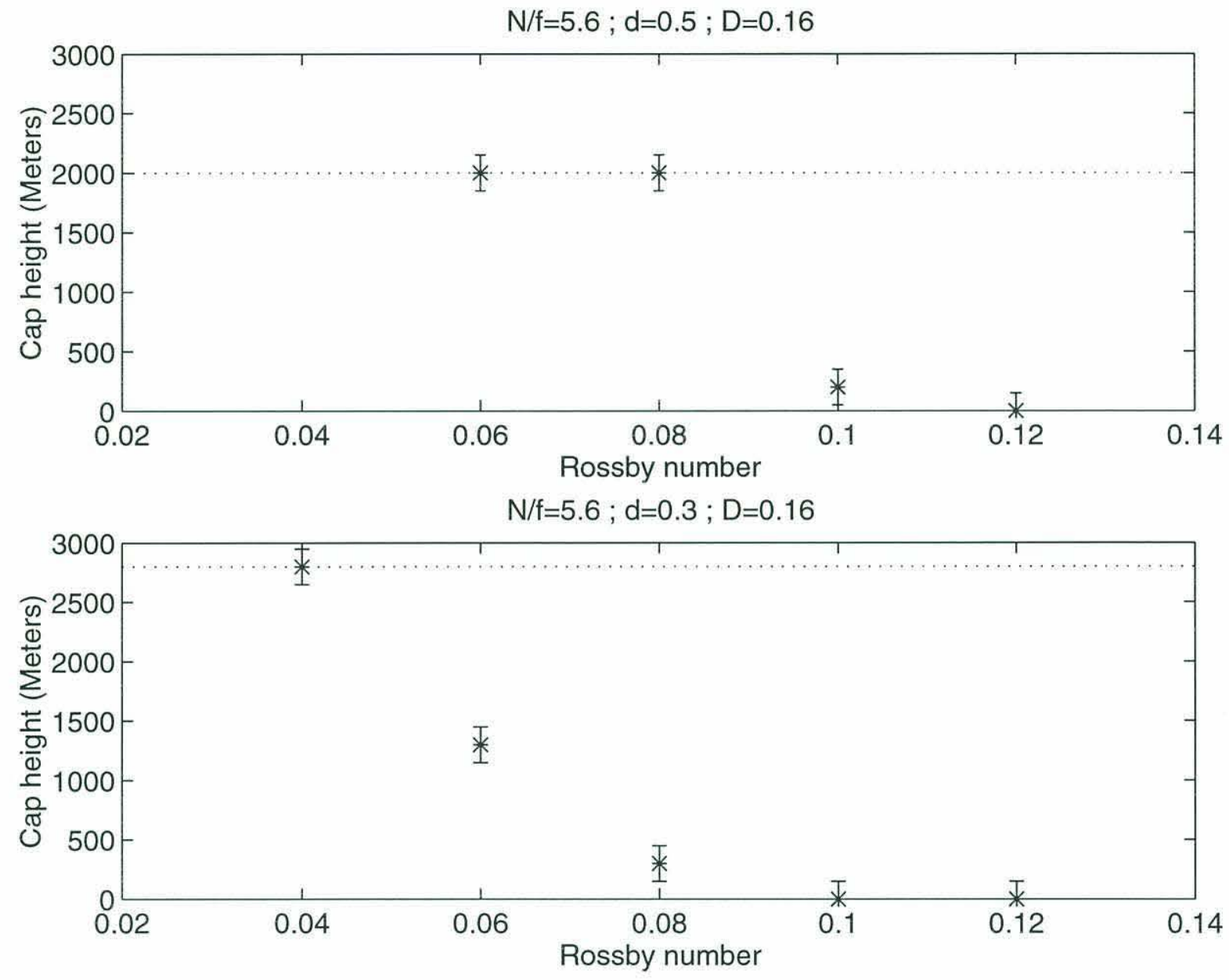

Figure 3.8: Taylor cap height dependence on Rossby number with stratification parameter $N / f=5.6$ and aspect ratio $D=0.16$. The upper and lower panels are for two different fractional heights, 0.5 and 0.3 respectively. The dotted line represents the maximum height attainable, namely the height corresponding to the upper boundary. 


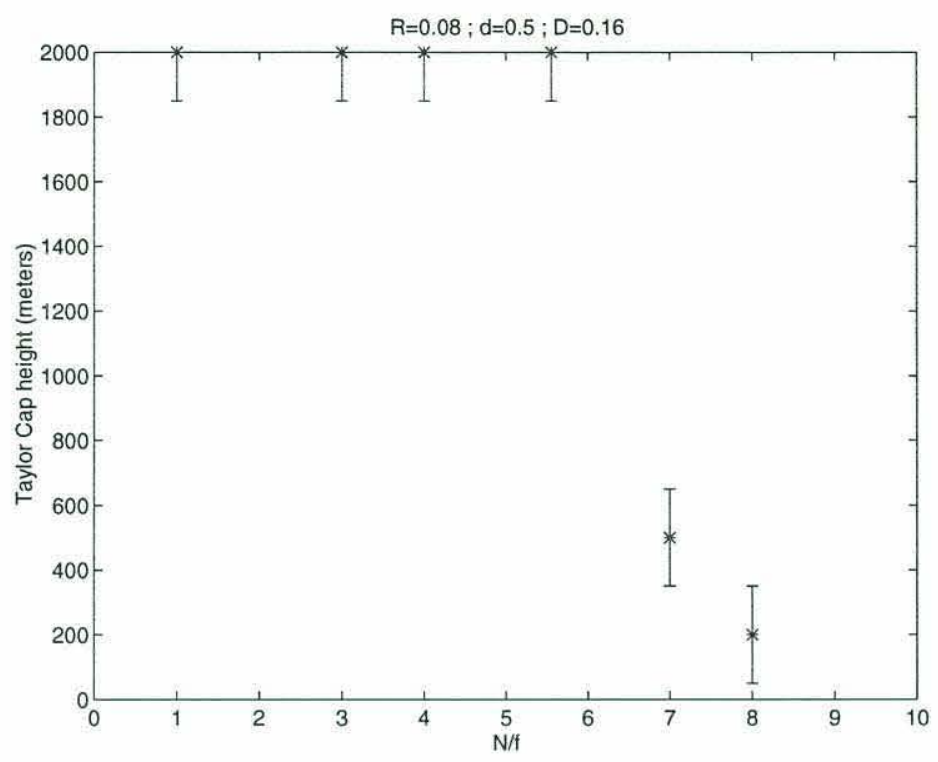

Figure 3.9: Taylor cap height dependence on the stratification parameter, $N / f$, with $R=0.08$, $\delta=0.5$, and $\Delta=0.16$. The maximum height attainable, the height corresponding to the location of the surface, is 2000 meters.

degree of stratification required to remove the recirculation completely was not found because of model instability problems at high stratification. However, extrapolation suggests that a value of $N / f$ of 10 is probably sufficient.

Finally, the dependence on seamount geometry is shown in figure 3.10 , which shows runs with various fractional heights with a Rossby number of 0.08 and $N / f$ of 5.6 . The critical height necessary for a Taylor cap to form above the level of the seamount peak is, in this case, between a value of 0.2 and 0.3 . The Taylor cap grows with increasing seamount height until, for a fractional height of 0.5 or higher, the Taylor cap reaches the surface. The dependence on aspect ratio has not been examined. All of the runs in this section have been performed with an aspect ratio of 0.16 , which corresponds to a background depth of 4000 meters and a seamount horizontal e-folding scale of 25 kilometers. 

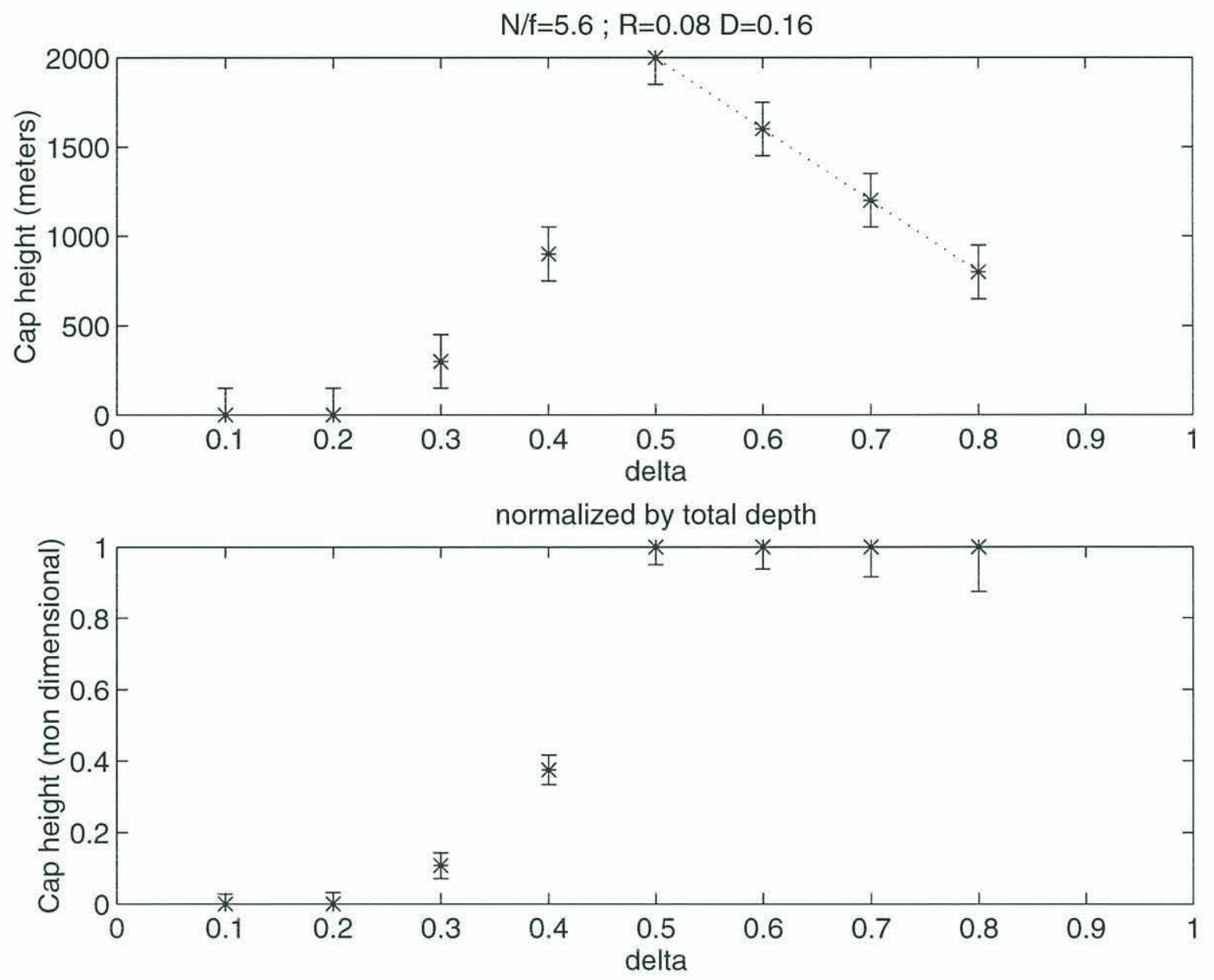

Figure 3.10: Taylor cap height dependence on fractional height, with $R=0.08, N / f=5.6$ and $\Delta=0.16$. The upper panel shows the real height in meters, with the dotted line representing the maximum height attainable, namely the height corresponding to the location of the upper surface. The lower panel shows the height of the Taylor cap normalized by the surface height for each different value of seamount fractional height. 


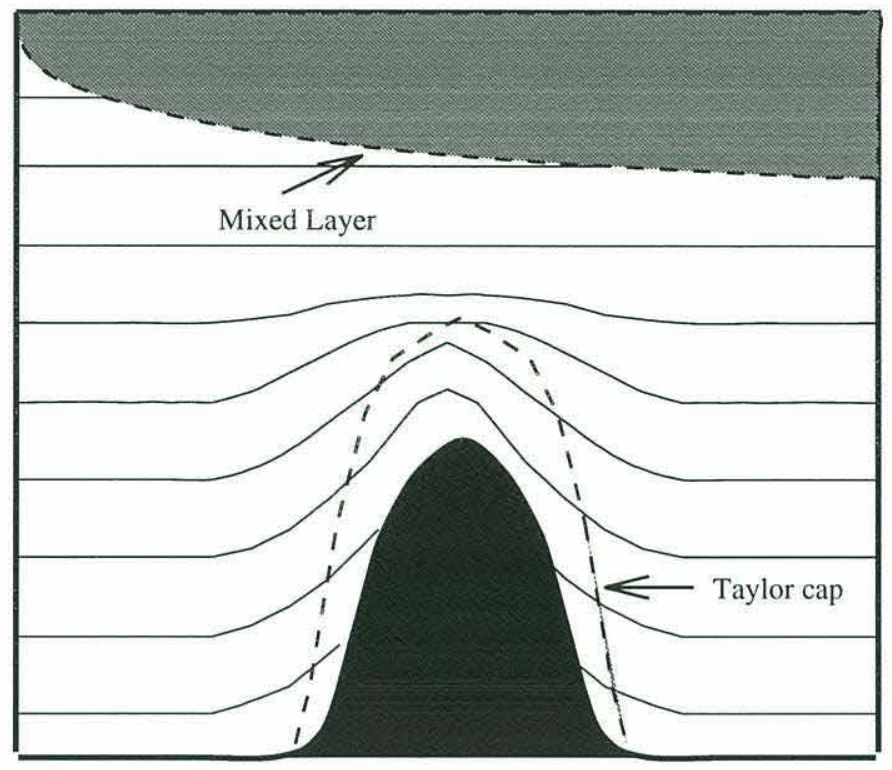

Figure 3.11: A schematic cross section running downstream through the center of the domain. The mixed-layer, with its square root dependence on downstream distance, and the Taylor cap, a region of topographically trapped fluid, are both shown. In this schematic the two regions do not overlap.

\subsection{Taylor Cap Mixed-Layer Interaction}

Given the dynamics of mixed-layer penetration depth and Taylor cap height in isolation, as presented in sections 3.2 and 3.3 respectively, it is now of interest to examine their interaction. A schematic cross section running downstream through the center of the domain is shown in figure 3.11 . This figure shows the mixed-layer as well as a region of trapped fluid over the seamount. In the figure the two regions do not intersect; thus the mixed-layer and Taylor cap dynamics do not affect each other. The key to having the Taylor cap precondition enhanced convection is for these two regions to overlap.

Consider run C4, a steady-state Taylor cap spin up over which surface negative buoyancy forcing has been applied, with associated nondimensional parameters as listed in Table 3.3. The Taylor cap for this run extends to a depth of 1200 meters below the surface. The expected mixed-layer depth 150 kilometers downstream from the inflow condition, calculated using equation 3.4 , is only 850 meters. Thus, one might expect no interaction between the two regions except for the fact that the mixed-layer depth in the 


\begin{tabular}{|l|c|c|c|c|}
\hline Run & $\begin{array}{c}\text { Nondimensional } \\
\text { Parameters }\end{array}$ & $\begin{array}{c}\text { Buoyancy } \\
\text { Forcing }\left(M^{2} / \mathrm{sec}\right)\end{array}$ & $\begin{array}{c}\text { Heat Flux } \\
\left(W / M^{2}\right)\end{array}$ & $\begin{array}{c}\text { convective Rossby } \\
\text { Number }\left(R_{N}\right)\end{array}$ \\
\hline C1 & $\mathrm{S} 2$ & $1.5 x 10^{-8}$ & 6 & $3.1 \times 10^{-2}$ \\
\hline C2 & $\mathrm{S} 2$ & $2.5 \times 10^{-8}$ & 10 & $4.0 \times 10^{-2}$ \\
\hline $\mathrm{C} 3$ & $\mathrm{~S} 2$ & $5.0 x 10^{-8}$ & 20 & $5.6 \times 10^{-2}$ \\
\hline C4 & $\mathrm{S} 2$ & $1.5 x 10^{-7}$ & 60 & $9.7 \times 10^{-2}$ \\
\hline C5 & $\mathrm{S} 2$ & $2.5 x 10^{-7}$ & 100 & $1.3 \times 10^{-1}$ \\
\hline M1 & $\begin{array}{c}N / f=5.556 \\
R=0.08 \\
\delta=\Delta=0\end{array}$ & $1.5 x 10^{-7}$ & 60 & $9.7 \times 10^{-2}$ \\
& & & \\
\hline
\end{tabular}

Table 3.3: Selected cooling runs. The surface forcing is listed in terms of the buoyancy forcing, equivalent heat flux, and the nondimensional convective Rossby Number, $R_{N}$. For the $\mathrm{C}$ series of runs, which are an extension of a Taylor cap spinup.

vicinity of the seamount, where there is a local deflection and retardation of the flow, will be significantly deeper than 800 meters due to the longer time a column of water has been exposed to the buoyancy flux. From figure 3.5, it is apparent that the mixed-layer around the seamount may penetrate as deeply as 1700 meters, which is well into the region of trapped fluid. Thus, the mixed-layer, interacting with the Taylor cap, could penetrate more deeply than elsewhere in the domain, leading to a convective chimney centered over the seamount flank.

A time history of mixed-layer depth in a slice through the center of the seamount is shown in figure 3.12. After only ten days of cooling the mixed-layer has reached its equilibrium depth of approximately 1000 meters. Away from the influence of the seamount, continued cooling does not increase surface density or deepen the mixed-layer perceptibly, because the cooling is completely balanced by horizontal advection of heat by the mean flow. Over the flank of the seamount, however, deepening continues to occur, creating a convective chimney.

For this particular run, C4, the chimney penetrates down to approximately 1700 meters depth by day 60 , after 40 days of surface forcing. Continued cooling, for this set of parameters, does not lead to further deepening, even in the vicinity of the seamount. 

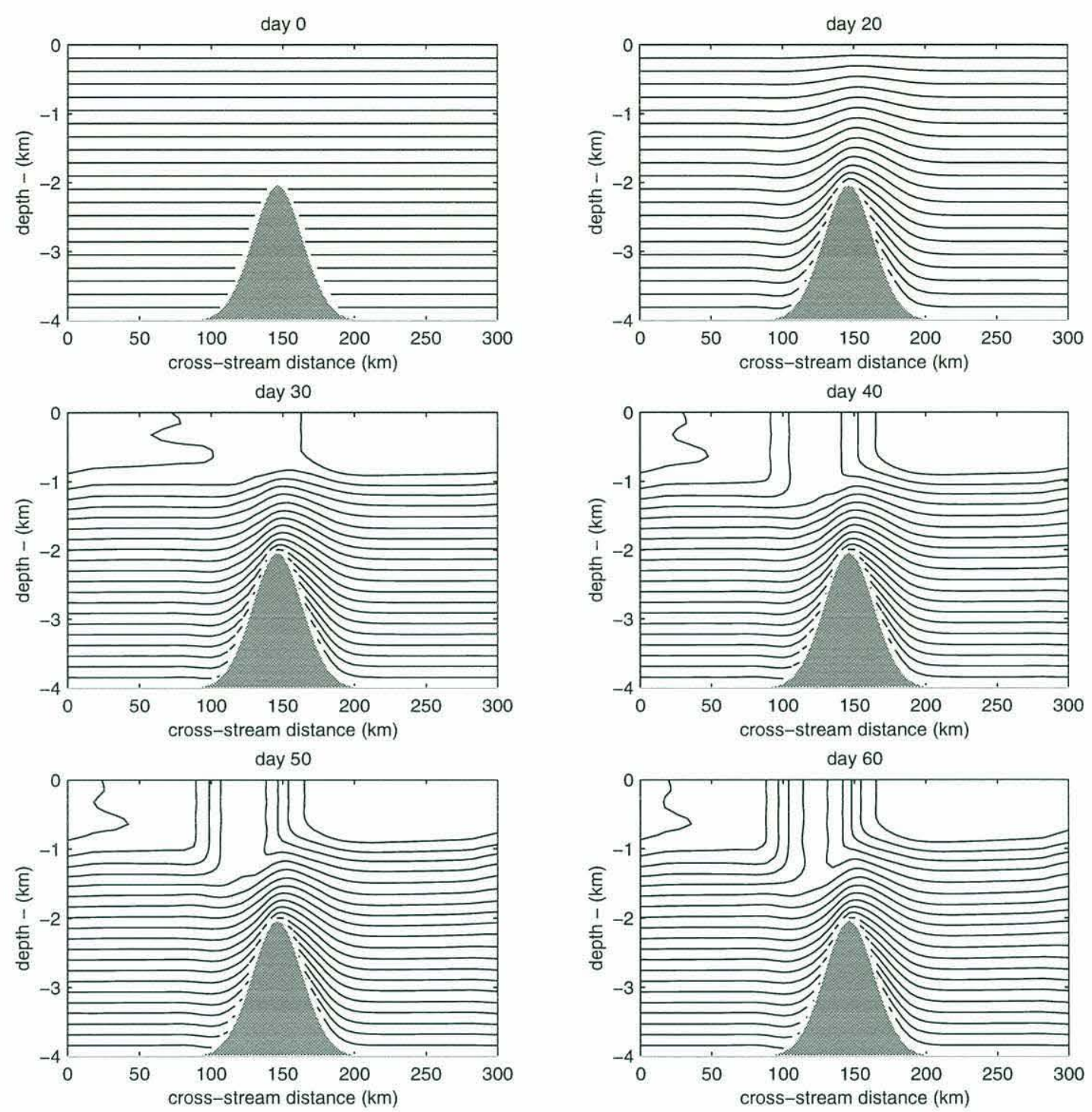

day 70
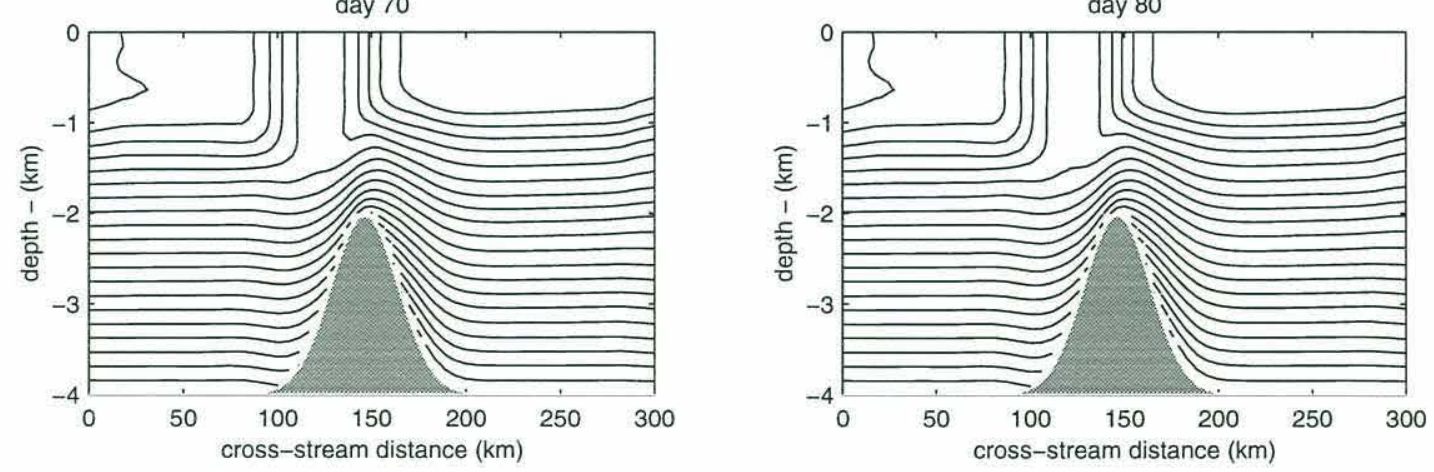

Figure 3.12: A time history of slices viewed from downstream located at a distance of 150 kilometers from the inflow for run C1. Surface cooling is initiated on day 20. 


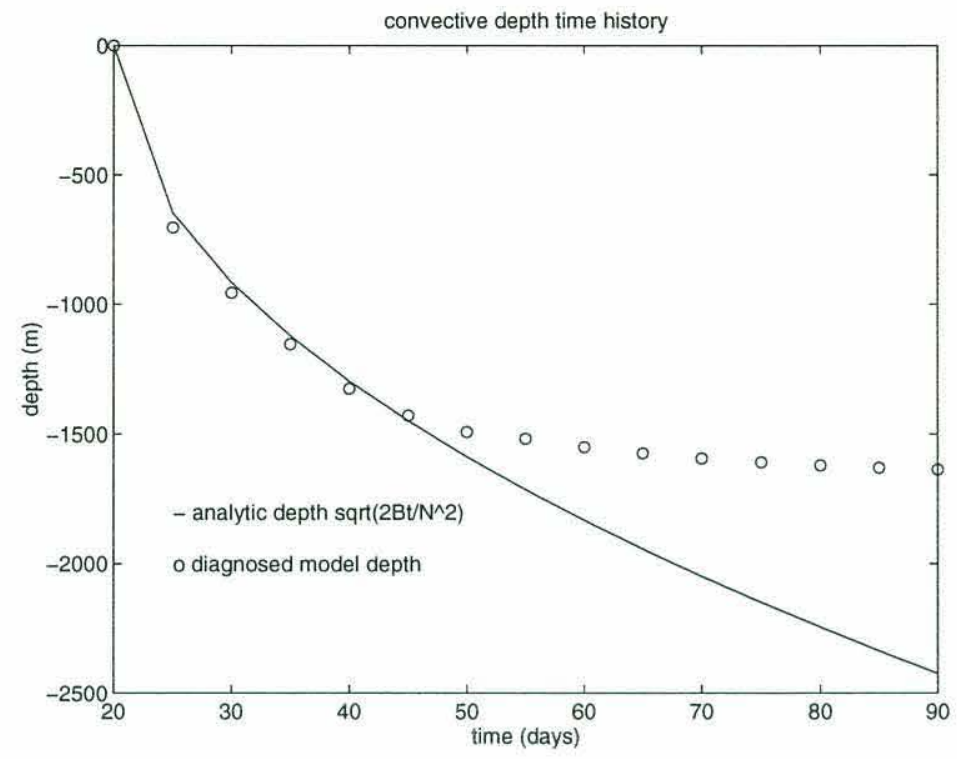

Figure 3.13: Maximum convective depth within the topographically trapped chimney for run $\mathrm{C} 1$ diagnosed at five day intervals. The analytic upper bound on one-dimensional, nonpenetrative convection limit is also shown.

A time series of maximum convective depth is shown in figure 3.13. For comparison, the one-dimensional penetration estimate from equation 3.3, using the total cooling time is also shown. Clearly, the convective chimney is deepening without being influenced by horizontal advection of heat until day 40. Continued cooling, between days 40 and 80 , is significantly retarded relative to the one-dimensional estimate, indicating lateral advection of heat into the chimney is partially balancing the surface heat loss. By day 80 , the chimney has reached a steady-state depth, with further cooling completely balanced by lateral advection.

In this case, the lateral advection of heat which shuts down the convective deepening is accomplished entirely by the mean flow across the horizontal temperature gradient associated with the convecting chimney. Because the region of trapped flow does not extend all the way to the surface, there is a significant downstream flow in the upper water column across the top of the chimney. This lateral flux of heat becomes large enough to balance the surface cooling over the top of the chimney as the horizontal temperature gradient increases. Note that the chimney initially forms because this mean 
flow advection of heat near the seamount is smaller than elsewhere in the domain, due to the reduced mean flow. However, as the chimney deepens the horizontal temperature gradient becomes sufficiently large for even this slow mean flow to lead to a significant horizontal advection of heat.

Since the mechanism for shutting down convection can differ for different sets of parameters, it is instructive to examine only the initial convective penetration depth in order to facilitate an understanding of the parameter dependence of the preconditioning alone. I have chosen, somewhat arbitrarily, to look at the extent of convective penetration after 20 days of cooling, at day 40 of run time.

The spatial variation of mixed-layer depth as determined from the surface density field at day 40 for run $\mathrm{C} 4$ is shown in figure 3.14 . There are at least three principal features to call attention to in this figure. First, away from the seamount, the square root dependence on downstream distance reminiscent of runs without topography is clearly visible. Second, the dense wake behind the seamount resulting from the retardation of the flow as discussed in section 3.2., is also apparent. Finally, as discussed above, the most pronounced deepening is centered on the right flank of the topography looking downstream, at the location of the Taylor cap. The parameter dependence of the penetration depth of this topographically trapped chimney is discussed in the next subsection.

\subsubsection{Parameter dependencies}

\section{Surface Buoyancy Forcing}

Perhaps the most obvious parameter to vary in order to investigate the deepening of the convective chimney is the buoyancy forcing itself. The upper panel of figure 3.15 shows a set of runs varying the buoyancy forcing over a range of nearly two orders of magnitude while holding all other parameters fixed. For reference, these runs, which are listed in table 3.3, are carried out with a Rossby Number of $0.08, \mathrm{~N} / \mathrm{f}$ value of 5.556, fractional height of 0.5 , and aspect ratio of 0.16 . Convective depths diagnosed from the model fields 


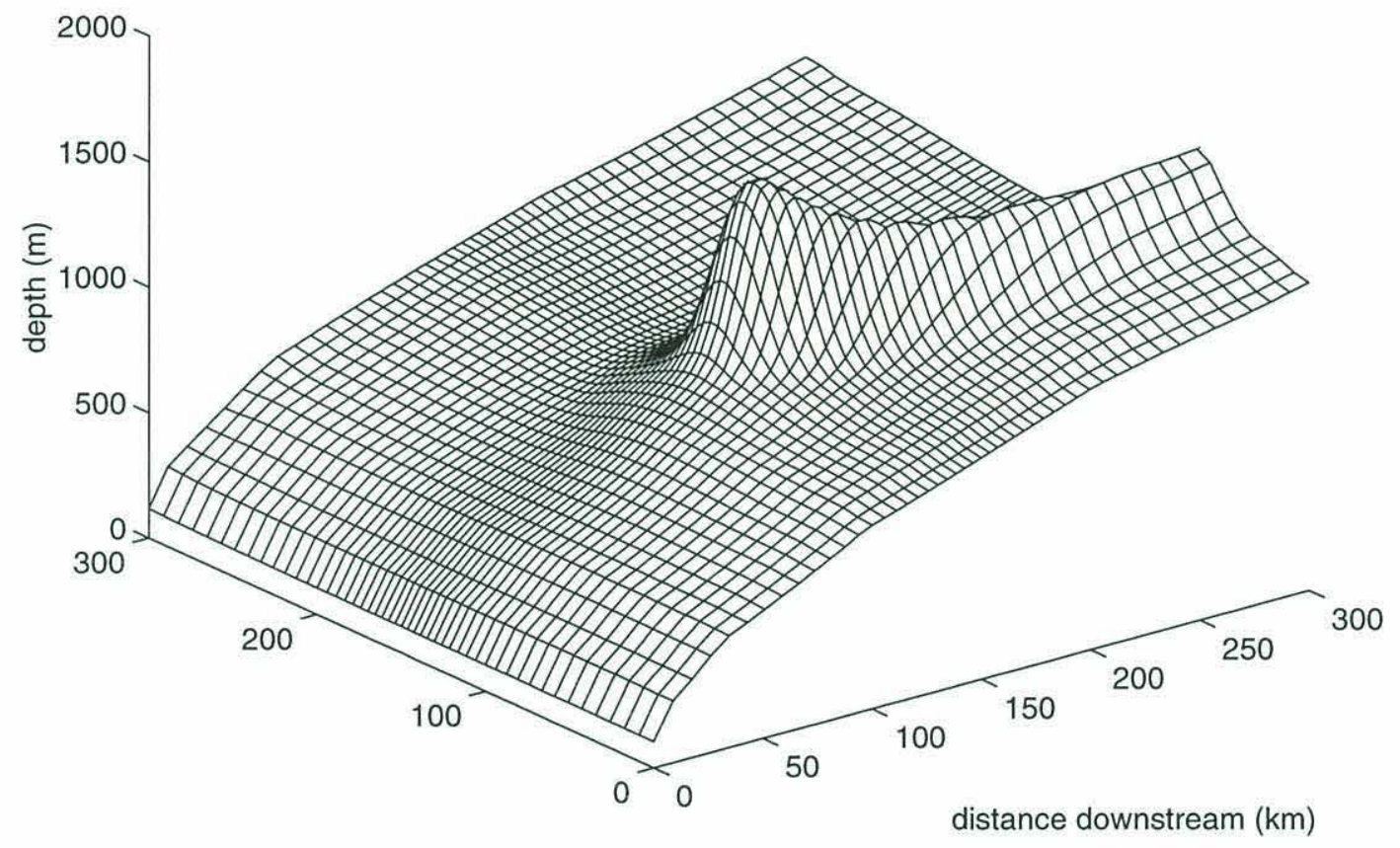

Figure 3.14: Mixed-layer depth diagnosed from the surface density field as a function of position within the domain for run $\mathrm{C} 1$ after 20 days of surface cooling. 


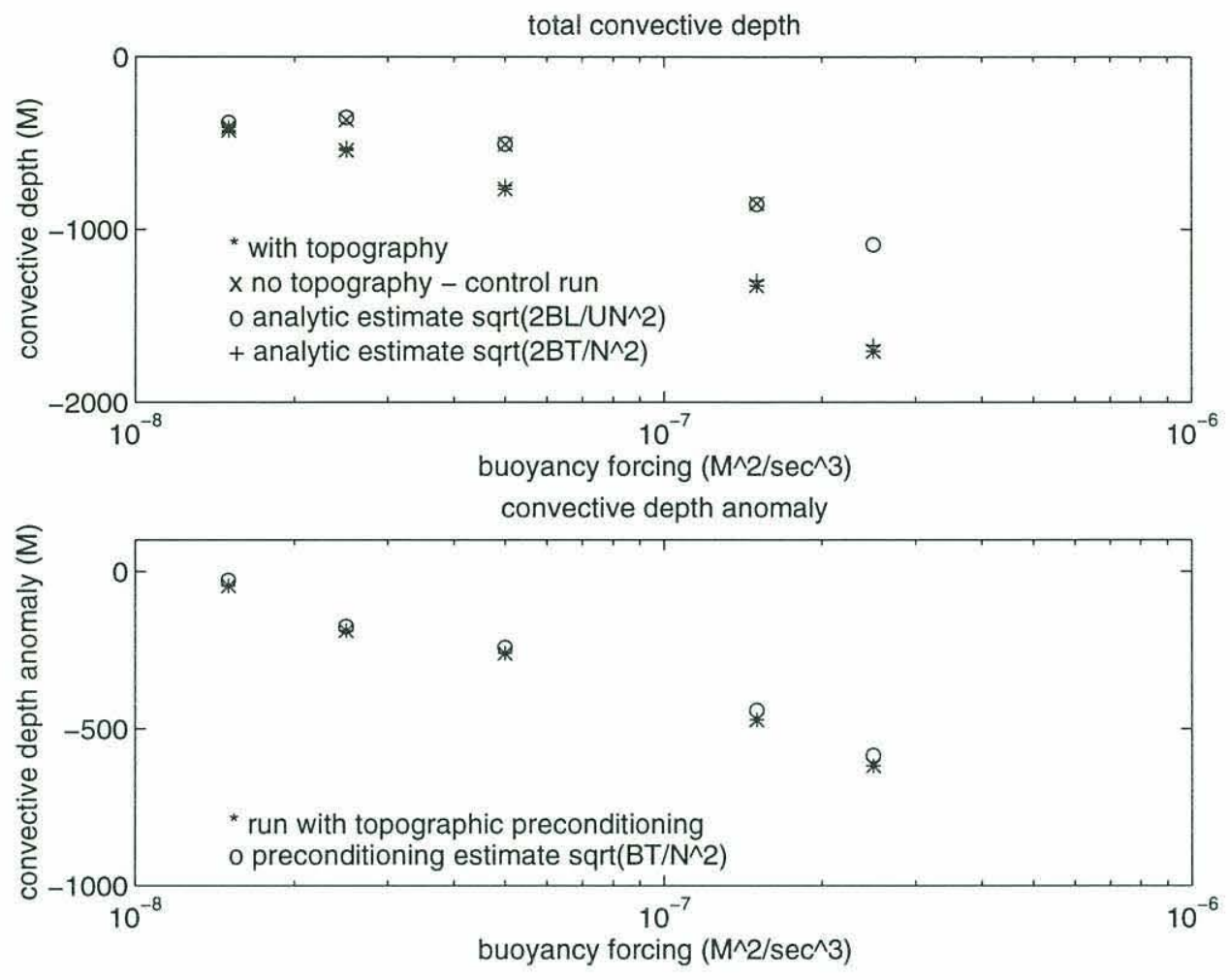

Figure 3.15: Convective depth as a function of buoyancy forcing. The upper panel shows the total convective depth reached with and without topographic preconditioning. The lower panel shows the difference between the two.

for cooling over Taylor cap spinup runs and runs without bottom topography, as well as analytic estimates for each type of run are plotted.

For the runs with topography, the maximum convective depth is diagnosed, regardless of its location in the domain. For comparison, the depth of penetration in the associated runs without topography is diagnosed at the same downstream distance at which the maximum convective depth occurs in the corresponding topographic run. The analytic estimates corresponding to the runs without topography are found using equation 3.4, the one-dimensional convection estimate with Lagrangian timescale based on the mean flow velocity and the location of the maximum convection in the topographic run. The estimates for the topographically preconditioned runs are from equation 3.3 , using the total cooling time of twenty days.

The run with the weakest surface forcing, C1, shows no preconditioning effect at 
all. For this run the maximum convective depth is found at the outflow, and is not significantly different from the mixed-layer depth found in the run with no topography. Clearly, in this case the surface mixed-layer is too shallow to interact with the region of trapped flow over the bottom topography. As a consequence, the presence of the topography does not precondition for enhanced convection. For all of the other runs, the deepest convection is located in an isolated chimney above the topography, and is significantly deeper than the mixed-layer depth measured in control runs. There is a tendency towards increased convection with higher buoyancy forcing in both the control and topographically preconditioned runs, as predicted by the dependence on square root of the buoyancy forcing in the analytic estimates.

In the lower panel of figure 3.15, the control convective penetration depth has been subtracted from the topographically preconditioned depth for each run. This figure shows that in addition to the increased convective penetration with increased surface forcing, there is also a distinct increase in the enhancement of the convective depth due to the presence of the topography.

\section{Seamount Fractional Height}

Shorter seamounts have their peaks deeper in the water column, and are thus more removed from surface forcing. In addition Taylor cap height has a strong dependence on the seamount height, with higher seamounts having taller associated Taylor caps. Figure 3.16 shows slices across the domain at 150 kilometers downstream distance for runs with four different seamount fractional heights after 20 days of cooling. The remaining nondimensional parameters are held constant at convective Rossby number of $9.7 \times$ $10^{-2}\left(B=1.5 \times 10^{-7}\right)$, Rossby number of $0.08, \mathrm{~N} / \mathrm{f}$ of 5.556 , and aspect ratio of 0.16 . Not surprisingly the smallest seamount has no noticeable preconditioning effect. In this case the seamount is too small to create a stagnation point (see figure 3.10) and, in addition, does not retard the near surface flow enough to significantly increase the time it takes particles in the mixed-layer to traverse the entire domain. Consequently, the mixed-layer 

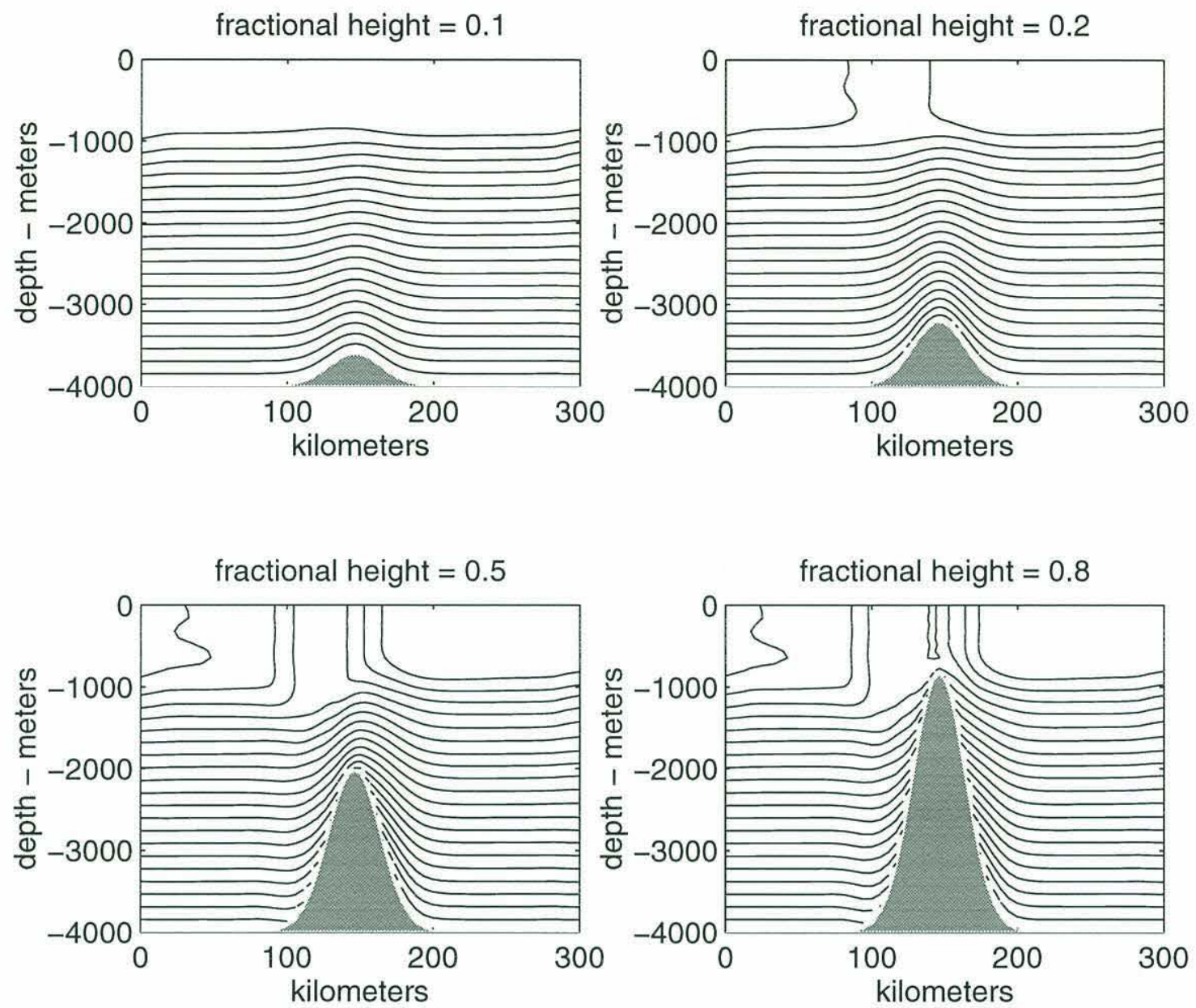

Figure 3.16: Vertical slices through the domain after 20 days of cooling at 150 kilometers downstream distance for runs with four different fractional heights, all other parameters held constant.

depth is not significantly different from that in control runs done without topography.

A slight increase in the fractional height to a value of 0.2 , still does not lead to an actual stagnation point in the flow field. However, in this case, the vertical shear in the horizontal velocity field is large enough to retard the mixed-layer flow somewhat, leading to a slight deepening of the mixed-layer in the vicinity of the seamount. The lower left frame in figure 3.16 shows the results from a run with a fractional height of 0.5 , well above the critical value for Taylor cap formation. This particular run, C4, was discussed earlier in this section, and the time series of slices at 150 kilometers downstream distance, 


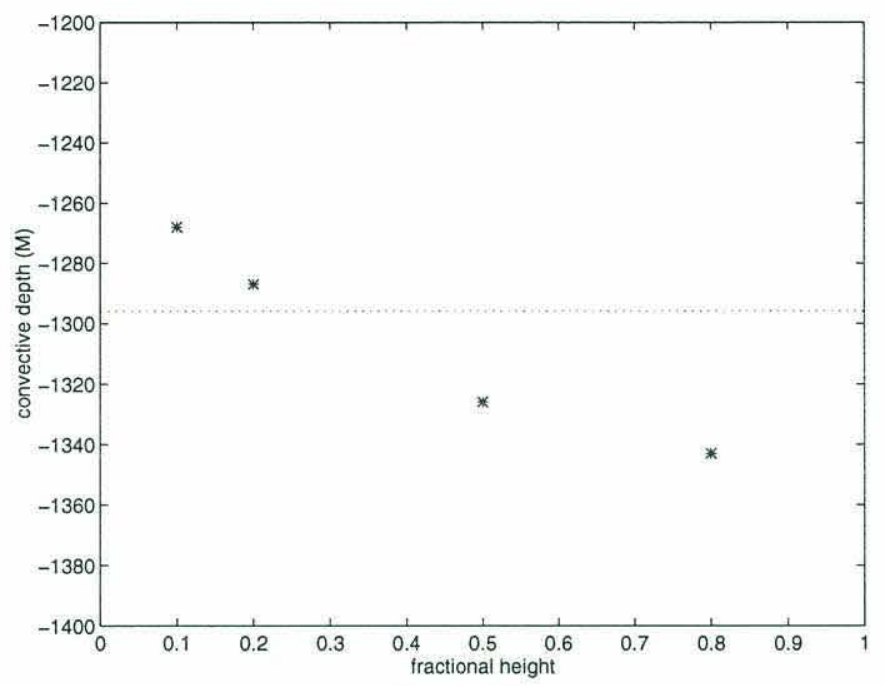

Figure 3.17: Convective depth as a function of seamount fractional height, holding all other parameters constant. The dotted line shows the analytical one-dimensional estimate of convection expected at that downstream distance neglecting topographic effects.

including this slice (at day 40), is shown in figure 3.12. As expected, a chimney appears over the flank of the seamount. Finally, for a fractional height of 0.8 , the preconditioning is again evident. In this case, the convective chimney is significantly wider than in the other runs indicating a larger horizontal extent to the Taylor cap. The chimney also penetrates all the way to the bottom of the water column (the top of the seamount), where the seamount crest rises to a depth of only 800 meters.

Figure 3.17 shows the maximum penetrative depth as a function of fractional height for each of these four runs. There is a tendency towards increased convection with increasing fractional height. The effect is not very strong, however, with the difference in convective penetration depth between the smallest and largest seamounts being an increase of only about 100 meters.

\section{Stratification Parameter}

Varying the stratification parameter, $N / f$, has a twofold effect. First, the higher stratification tends to reduce the depth of the mixed-layer. Second, increased stratification 
also reduces the height of the Taylor cap. Both of these effects tend to decouple the surface mixed-layer and the deep topographically trapped flow. Thus, a strong dependence on the stratification parameter is expected. Figure 3.18 shows a set of slices at 150 kilometers downstream for runs with varying values of $N / f$. The remaining parameters are held constant with a convective Rossby Number of $9.7 \times 10^{-2}$, Rossby Number of 0.08 , fractional height of 0.5 , and aspect ratio of 0.16 . Again, the slices are shown after 20 days of cooling. The same contour level is used in each slice, such that the number of isopycnals shown gives an impression of the magnitude of the stratification in each run. For reference, the run with intermediate stratification in this comparison is run C4, referred to in the other parameter sensitivity sections.

Figure 3.19 shows the depth of penetration of the convecting chimney versus stratification parameter. Clearly dependence on the stratification parameter is quite strong. Varying $N / f$ by less than a single order of magnitude roughly maps out the difference between a run with almost no topographic preconditioning whatsoever and one in which the chimney penetrates to the bottom over the flank of the seamount.

\subsection{Conclusions}

In regions of the ocean where open ocean deep convection occurs there is often a large discrepancy between the large spatial scale (100's to 1000's of kilometers) on which surface buoyancy forces act and the smaller scale of convective chimneys (10's to 100's of kilometers) which ventilate the deep water. Topographic influence is one of several ways in which regions of the ocean can be preconditioned to preferentially convect. Mean flow past isolated topography leads to at least two means by which the water column above the topography can be thought of as preconditioned. First, the isopycnals tend to be domed above the seamount. Second, if a Taylor cap forms over the topography, the isolation of the local fluid from the effects of horizontal advection maximizes the potential convective deepening locally because of the lack of lateral fluxes of heat into the region 

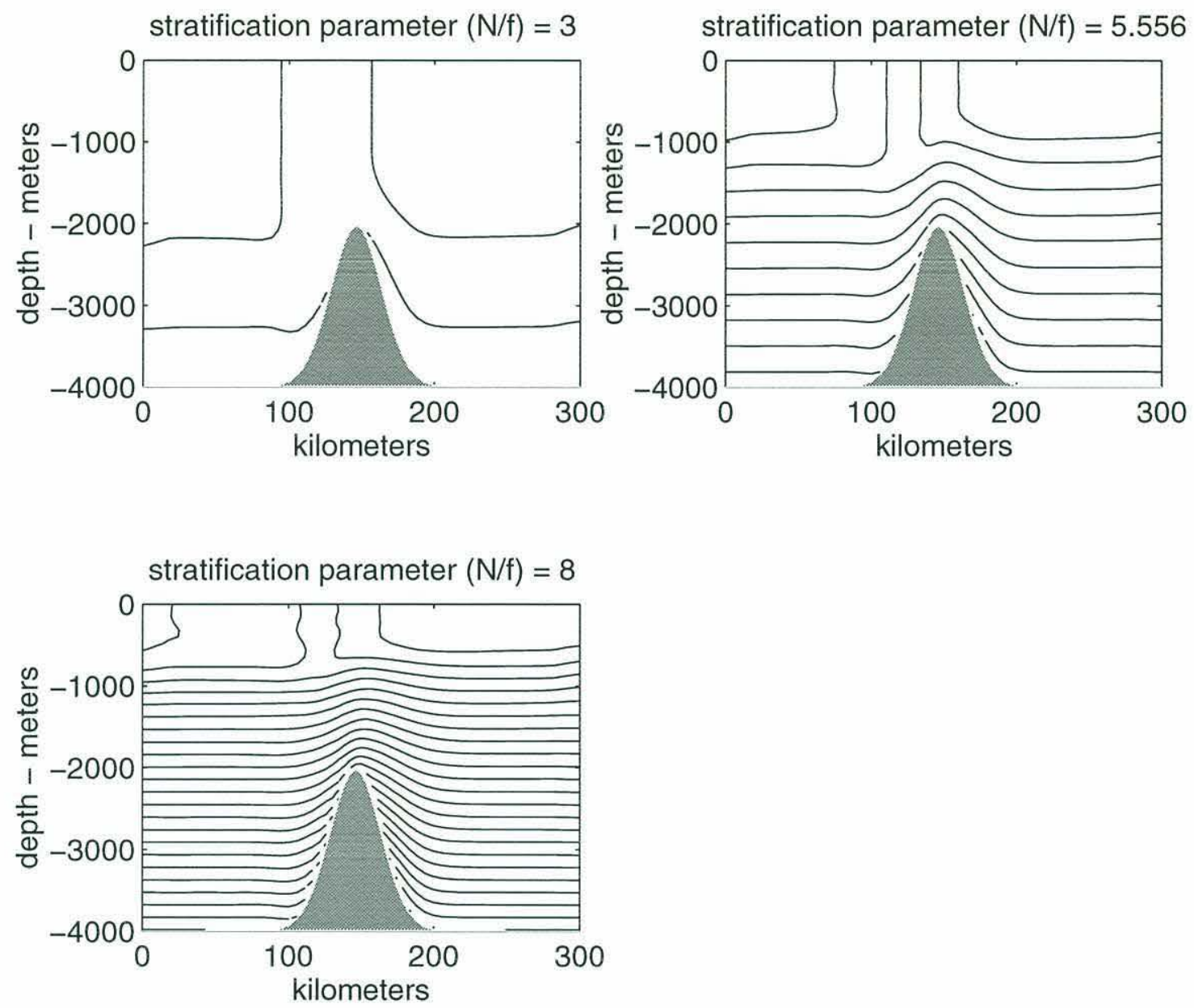

Figure 3.18: Vertical slices through the domain at 150 kilometers downstream distance for runs with three different values of the stratification parameter, $N / f$, with all other parameters held constant. The contour interval is held fixed, giving an indication of the different magnitudes of the stratification. 


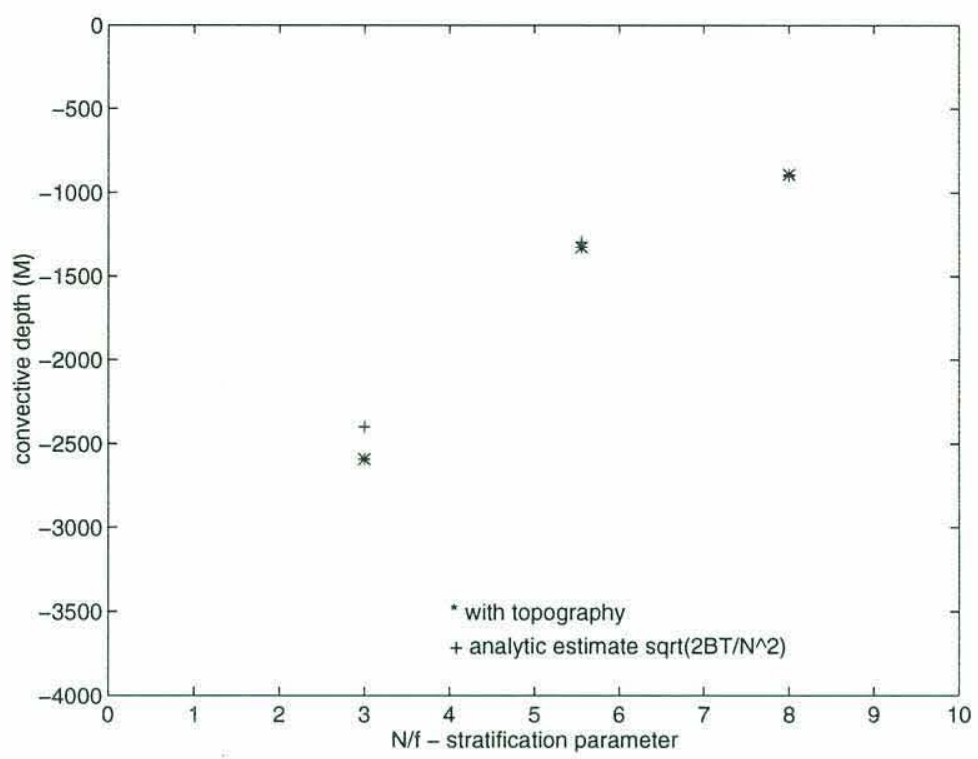

Figure 3.19: Convective depth as a stratification parameter, holding all other parameters constant.

of trapped fluid.

In order to study these topographic effects, the SPEM model is configured as an open channel, with a barotropic mean flow impinging on a Gaussian seamount. Initially, a steady-state Taylor cap is spun up. A uniform surface cooling is then applied as a surface boundary condition. The surface cooling leads to a mixed-layer with a steadystate depth which goes as the square root of the distance from the inflow of uniformly stratified water. The mixed-layer steady-state is achieved when the surface buoyancy flux is everywhere balanced by the lateral advection of heat associated with the mean flow bringing in stratified water from outside the computational domain.

In the vicinity of the topography, the retardation of the mean flow and trapping of fluid in the Taylor cap both tend to increase the mixed-layer depth, primarily because of the increased time particles spend in the domain under the influence of the surface forcing. A convective chimney appears over the right flank of the seamount, looking downstream, when the Taylor cap reaches up into the surface mixed-layer. In addition a dense wake extends out behind the topography with water which, although not trapped over the topography, does slow down significantly, being exposed to a much longer period 
under the surface cooling than water away from the topographic influences. The doming of isopycnals over the seamount appears to be unimportant for these preconditioning experiments.

Five nondimensional parameters are used to investigate the behavior of this system. Four parameters, the Rossby number, seamount aspect ratio, seamount fractional height and stratification parameter completely define the steady-state Taylor cap problem. The addition of surface buoyancy forcing requires the addition of a fifth parameter, the convective Rossby number. The primary requirement for a deep convecting chimney to develop over the topography is that the Taylor cap reach high enough in the water column to interact with the surface mixed-layer. The mixed-layer depth is calculated as the depth in the initial uniform stratification profile associated with the surface density at a given point. The Taylor cap height is determined through the use of two diagnostics, the location of a flow reversal in the horizontal velocity field and the distribution of a passive tracer.

Increasing Rossby Number and stratification parameter both tend to reduce the surface mixed-layer depth and also reduce the height of the vertical extent of topographic influence. Thus, the extent of convective preconditioning decreases as Rossby Number and stratification parameter increase. Buoyancy forcing directly affects only the surface mixed-layer depth. If the buoyancy forcing is low, the mixed-layer does not penetrate deep enough to interact with the topographically trapped flow, and there is no preconditioning effect. However, there is an increasing convective depth anomaly, defined as the depth of convection compared to a run with no topography, as a function of buoyancy forcing. The importance of fractional height is mostly due to the fact that higher seamounts are more likely to interact with the surface mixed-layer, all other parameters being held constant. However, the effect of varying fractional height is generally small within the range of the other parameters tested. The effect of varying the seamount aspect ratio was not investigated.

These parameter sensitivity studies have all been carried out after a somewhat arbi- 
trary 20 days of cooling. In the following two chapters two important qualifications to the topographic preconditioning process are addressed. In chapter 4, a background stratification which varies more realistically as a function of depth is included. The mechanisms which operate to shut down convective deepening, despite continued surface cooling, are discussed in Chapter 5. 


\section{Chapter 4}

\section{Nonuniform Stratification}

In section 3.1.1 the expected depth of convection for one-dimensional, non-penetrative convection into a constant background stratification is shown to be a simple function of the stratification, and the magnitude and time of cooling. The relationship is reproduced here for reference:

$$
d=\sqrt{\frac{2 B T}{N^{2}}}
$$

In the topographically preconditioned chimneys with constant background stratification studied in chapter 3 , the initial convective depth within the chimney is well approximated by equation 4.1. Despite the fact that the derivation of the analytic scaling assumes a constant value for $\mathrm{N}$, there is agreement between chimney depth in these model runs and the analytic scaling. This agreement implies that the deviation from constant background stratification due to doming of isopycnals over the seamount does not have a great effect on the depth of convection. Yet doming of isopycnals is a ubiquitous paradigm for topographic preconditioning. What is the reason for this apparent contradiction?

In this chapter, I investigate the preconditioning role of domed isopycnals in the context of nonuniform background stratification, first from a simple analytic viewpoint and then with the numerical model. Section 4.1 introduces how domed isopycnals can be expected to act as a preconditioning mechanism. In section 4.2 , I describe a simple analytic solution for the depth of non-penetrative convection into an exponential stratification and 
compare it with results from a run with the SPEM initialized with the same exponential stratification. In section 4.3 this analytic solution is used to demonstrate the drastic increase in the depth of convection that the nonuniform stratification allows. Finally, in section 4.4, some topographic preconditioning runs with an exponential background stratification are shown and compared with the constant stratification runs discussed in Chapter 3.

\subsection{Domed Isopycnals}

Imagine a region where isopycnals are domed in such a way as to maintain a constant buoyancy frequency. In this case it is necessary for some isopycnals to outcrop at the surface, as shown schematically in figure 4.1(a). A given surface forcing will lead to identical depths of convection, as given by equation 4.1, throughout the domain. However the maximum surface density, corresponding to the maximum density of ventilated water, will always be located over the region of isopycnal doming, as it was before the onset of cooling. This scenario for domed isopycnals can be thought of as lifting the background stratification, and requires the buoyancy content associated with the part of the stratification that has been lifted out of the water altogether to have been removed by some previous mechanism. I refer to this as the "lifting" mechanism for doming isopycnals. One preconditioning scenario in which the lifting paradigm is appropriate is in a remnant of a convective chimney left over from a previous cold event or even from the previous year. In such a region isopycnal outcropping has already occured, removing surface buoyancy forcing and leaving an adjusted final state similar to that in figure 4.1 (a).

A second scenario to consider is a region of uplifted isopycnals beneath a constant surface temperature as shown in figure 4.1(b). In this case, none of the isopycnals outcrop until the cooling commences. The near surface buoyancy frequency is greater in the region of isopycnal doming than elsewhere. Since the buoyancy frequency is not a constant, 
(a)

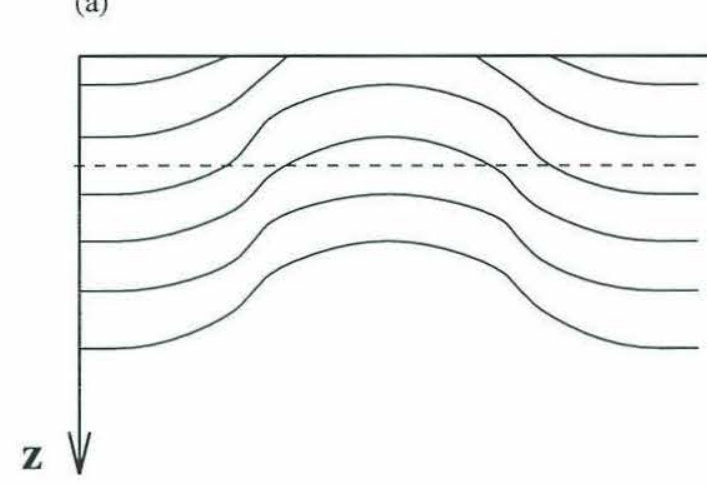

(b)

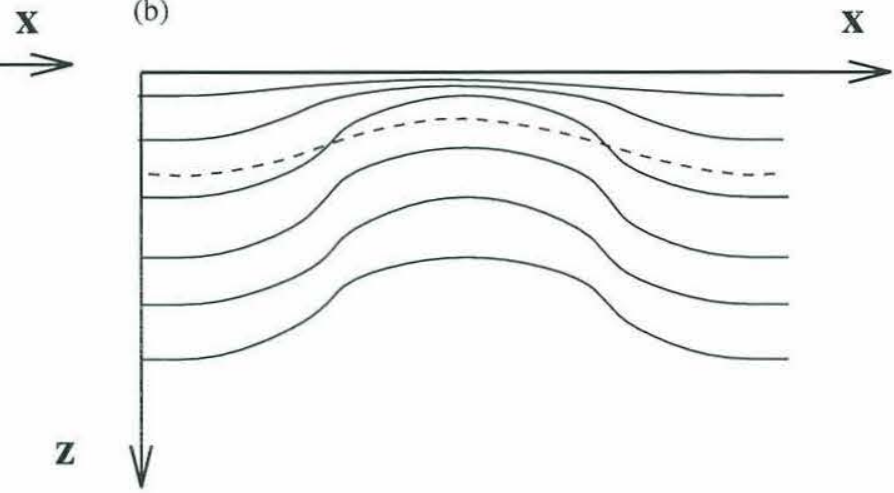

Figure 4.1: Schematics of a hypothetical region of domed isopycnals: (a) maintains a constant buoyancy frequency, requiring some isopycnals to outcrop, while (b) maintains a constant surface temperature, leading to locally enhanced buoyancy frequency near the surface. The dotted line is an estimate of the non-penetrative, one-dimensional convective penetration depth after some arbitrary amount of cooling. Note that in both cases the densest surface water is located in the region of doming, although in neither case has convection penetrated deeper in that region.

but a function of depth, and equation 4.1 is not strictly valid. However, the inverse dependence on the local buoyancy frequency is still likely to determine penetration depth, leading to deeper penetration into the water column away from the domed isopycnals where the stratification is weaker. However, the maximum surface density will again be located above the region of doming. This doming scenario, which can be thought of as a "squeezing" of the isopycnals, does not require any isopycnal outcropping prior to the onset of cooling. This squeezing paradigm for doming is consistant with preconditioning mechanisms such as baroclinic instability of a zonal flow or flow over topography, as discussed in this thesis.

In neither of these cases is convection expected to penetrate more deeply in the region of domed isopycnals. Nevertheless, the region can be considered preconditioned since the densest surface water is formed there. Of course, the newly formed dense water can always flow along isopycnals to greater depths away from the preconditioned area without requiring further cooling. However, in most regions of open ocean convection in the real world, the convective chimney does penetrate to a much greater depth than the mixed-layer depth in surrounding waters. This implies that domed isopycnals alone can 


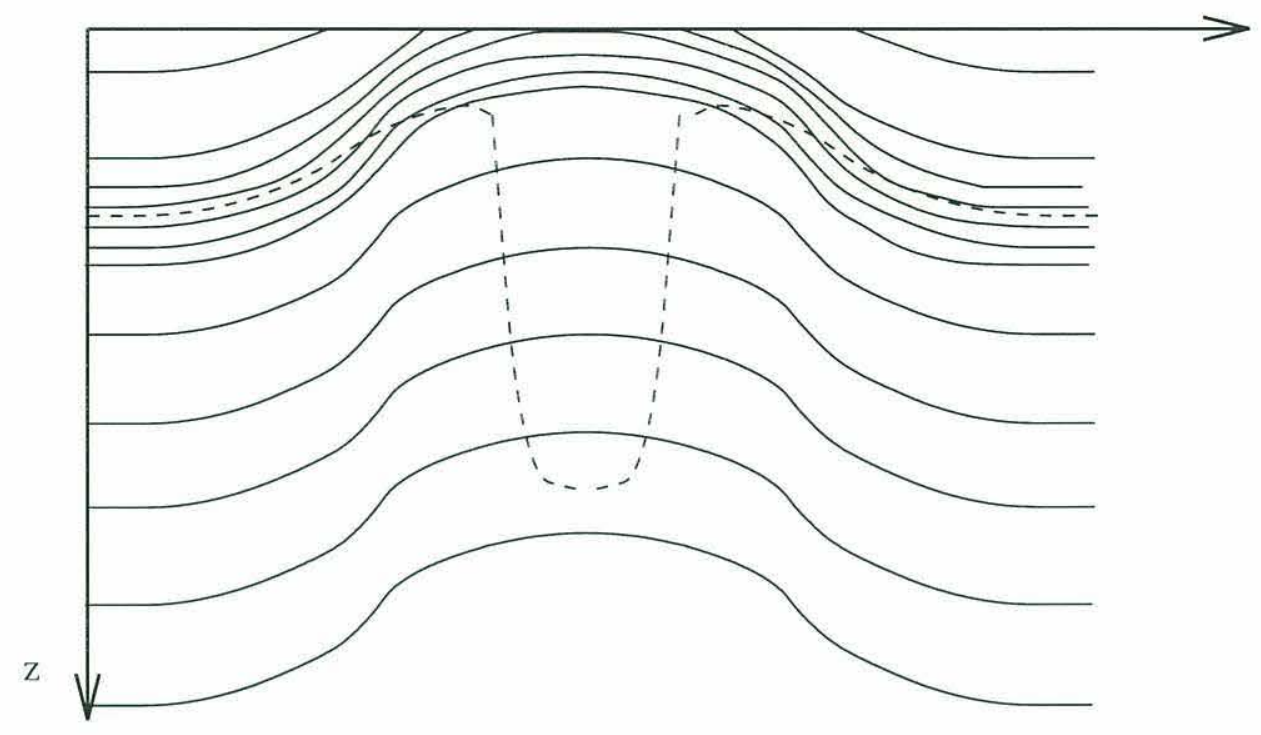

Figure 4.2: A schematic of a more realistic, nonuniform stratification consisting of weakly stratified surface region and abyss, separated by a pycnocline. The dotted line represents the non-penetrative, one-dimensional convective penetration depth. In this case both the densest surface water and the deepest convection are located in the region of isopycnal doming.

perhaps not fully explain the preconditioning effect.

The real ocean does not have a constant background stratification. More realistic stratification generally consists of a weakly stratified surface layer overlying a pycnocline all on top of a weakly stratified deep interior. A schematic of one such stratification is shown in figure 4.2. In this case, the preconditioning effect of domed isopycnals is most readily apparent. In order to convect deeply, significant cooling is first required to punch through the strongly stratified pycnocline. However, once the lower layer is ventilated, its weak stratification allows rapid convective deepening. The proximity of the pycnocline to the surface in the domed isopycnal region ensures that this is where convection will first penetrate. Consequently, domed isopycnals preselect a region to convect most deeply, not just to a denser isopycnal value, for this more realistic stratification. These heuristic arguments suggest that only after allowing for the presence of a pycnocline can local doming of isopycnals precondition for convective chimney formation. This possibility is investigated in detail in this chapter. 


\subsection{A One-Dimensional Analytic Model}

In order to make some progress analytically, one simple and commonly used method for representing the effect of a surface-trapped pycnocline is through the use of an exponential density profile:

$$
\rho(z)=K\left(1-e^{z / \sigma}\right)
$$

Here $\rho$, the density anomaly, always has a surface value of zero while at great depth the density approaches the value $K$. The e-folding scale, $\sigma$, is a measure of the degree to which the pycnocline is surface-trapped. Three different exponential density profiles, all chosen to have approximately the same surface to bottom density difference as a constant stratification run with a value of $N / f=5$, are shown in the left panel of figure 4.3. The right panel shows the buoyancy frequency as a function of depth associated with each of these density profiles. As in section 3.1 the penetration depth of a uniform negative surface buoyancy flux can be calculated by equating the time-integrated density flux with the difference between the vertically integrated original heat content and that of the uniform profile after convection has occurred.

Two schematics are shown in figure 4.4. The schematic (a) shows the "lifting" mechanism for ispopycnal doming and (b) the "squeezing" mechanism. In both cases the shaded area represents the total heat content difference between the original exponential stratification and the uniform profile which exists after convection has occurred. Two profiles are shown in each schematic, one represents some background exponential stratification and the other the stratification as it might appear in the region of domed isopycnals. The measures of convective depth for each profile are denoted $\mathrm{d} 1$ and $\mathrm{d} 2$ respectively. Clearly, for the same amount of surface cooling, conserving the area of heat content loss due to convection, penetration depth will be much greater in the region of doming. This is true for the lifted density profile in (a) as well as for the squeezed profile in (b). In contrast, figure 4.5 shows a similar set of profiles, one background and one in the region 

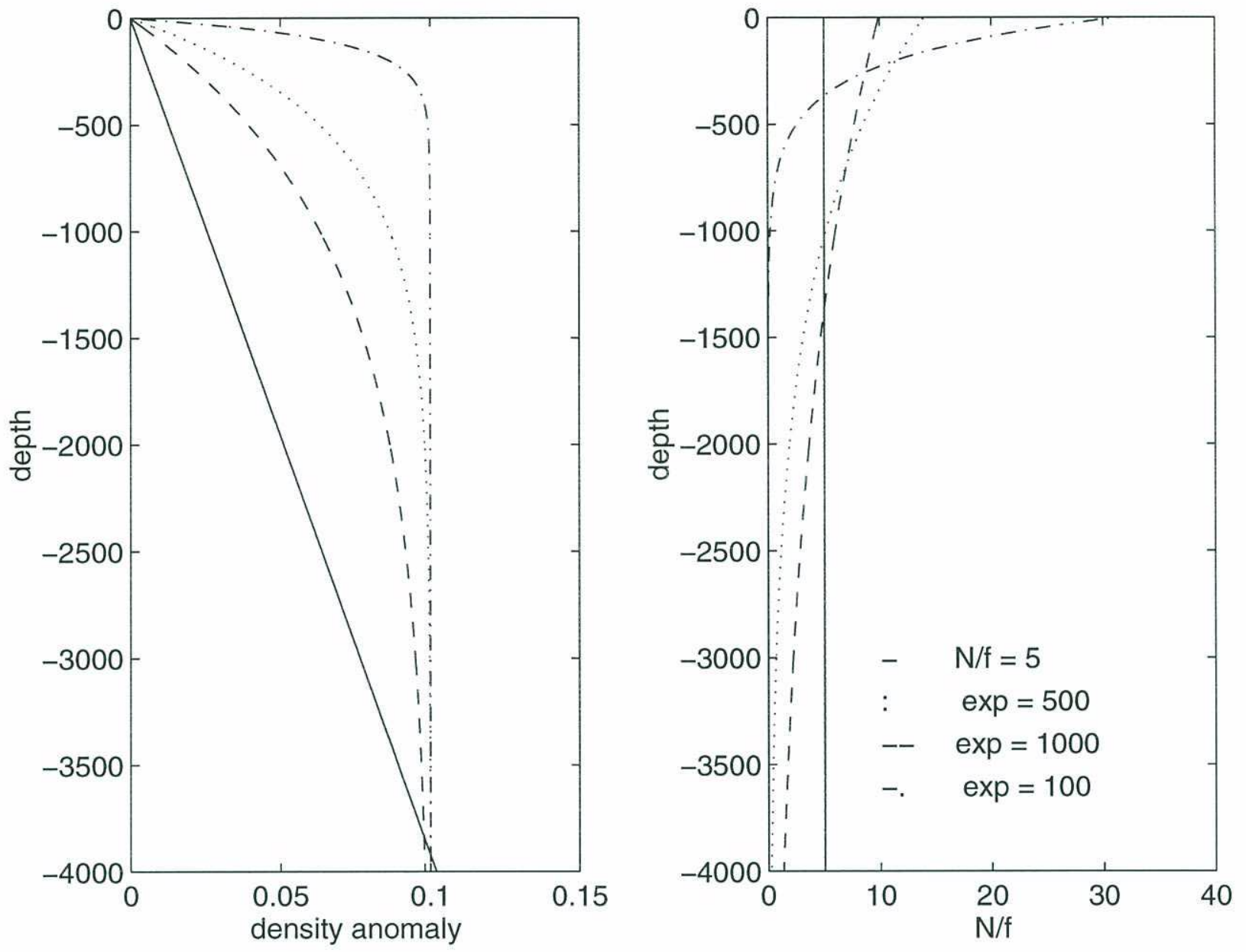

Figure 4.3: The left hand panel shows three exponential profiles, with e-folding scales of 100, 500 and 1000 meters, all chosen to have the same surface to bottom total density difference as a constant profile with $N / f=5$. The right hand panel shows the buoyancy frequency profiles associated with these exponential density profiles, again referenced to the constant stratification $N / f=5$ case. 
(a)

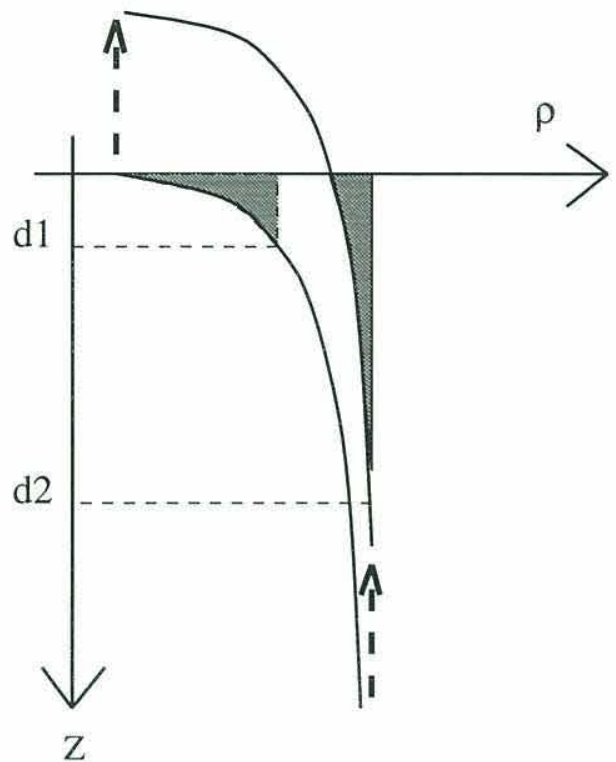

(b)

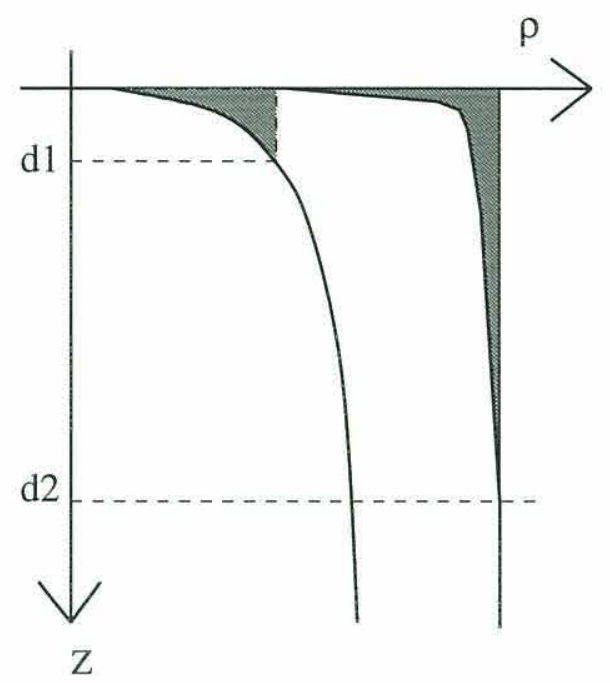

Figure 4.4: This figure shows set of profiles, one background and one in a region of doming. Panel (a) represents the doming with a lifting of the profile (as denoted by the heavy dashed arrows), whereas panel (b) employs a squeezing of the isopycnals. The shaded area represents the total heat content difference between the original exponential stratification and the uniform profile which exists after convection has occurred. The measures of convective depth for each profile, given some uniform amount of surface cooling, are denoted $\mathrm{d} 1$ and $\mathrm{d} 2$ respectively. Clearly, conserving the area of heat content loss due to convection, penetration depth will be much greater in the region of doming irrespective of whether the doming is produced by lifting or squeezing of the exponential profile.

of doming, for a uniuform stratification. In the case of lifted isopycnals, the two depths of penetration, $\mathrm{d} 1$ and $\mathrm{d} 2$, are identical, as expected given equation 4.1. The penetration depth into the squeezed profile is actually less than in the background profile because of the higher near surface stratification in the region of doming.

For the case of exponential stratification, a solution for the convective depth can be obtained by setting the total surface density flux equal to the change in the total density of the water column during convection, giving:

$$
B T=\frac{g}{\rho_{0}} \int_{d}^{0}(\rho(d)-\rho(z)) d z .
$$

Substituting in for $\rho(d)$ and $\rho(z)$ from equation 4.2 gives,

$$
B T=\frac{g}{\rho_{0}} \int_{d}^{0}\left(K\left(1-e^{d / \sigma}\right)-K\left(1-e^{z / \sigma}\right)\right) d z .
$$




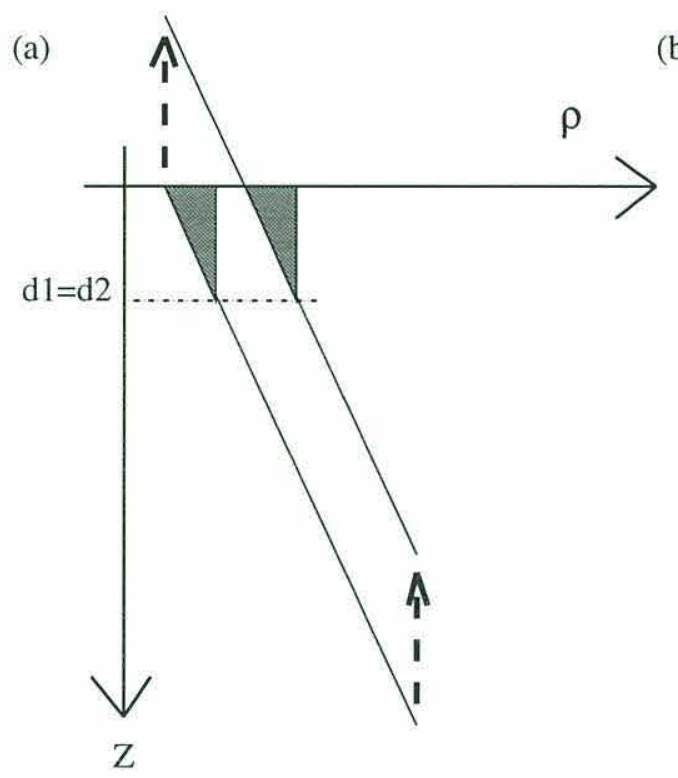

(b)

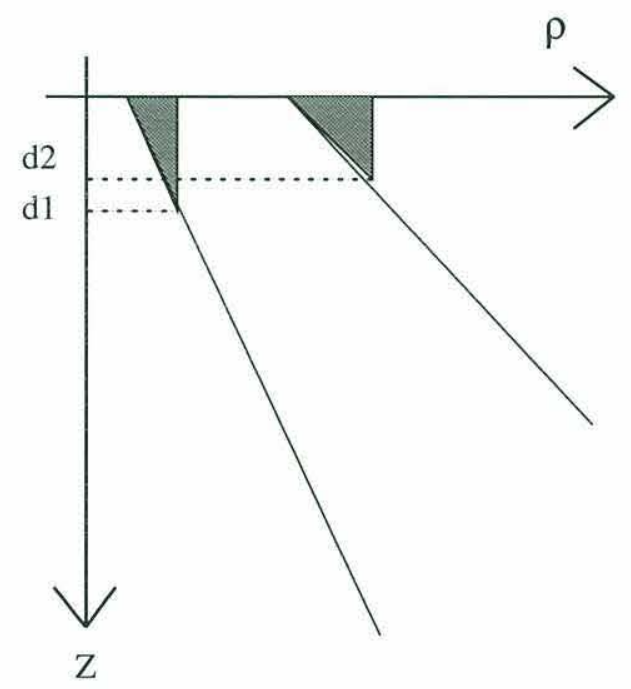

Figure 4.5: This figure shows set of profiles, one background and one in a region of doming. Panel (a) represents the doming with a lifting of the profile (as denoted by the heavy dashed arrows), whereas panel (b) employs a squeezing of the isopycnals to represent the doming. In this case, unlike for the exponential profile, depth of penetration in the region of doming, $d 2$, are not enhanced relative to the background level, d1. As expected, constant stratification precludes chimney formation in regions of domed isopycnals. 
Which simplifies to:

$$
B T=\frac{g}{\rho_{0}} K \int_{d}^{0}\left(e^{z / \sigma}-e^{d / \sigma}\right) d z .
$$

This integral is a trivial one; indeed, that is why the exponential stratification was chosen in the first place. Finally, an implicit analytical expression for convective penetration depth is obtained:

$$
B T=\frac{g}{\rho_{0}}\left(K \sigma+K d e^{d / \sigma}-K \sigma e^{d / \sigma}\right) .
$$

Although an explicit formula for the convective depth, $d$, is not achievable, iterative solutions of this implicit formulation are readily obtainable.

As an example, consider the stratification with e-folding scale, $\sigma$, of 500 meters and surface to bottom density difference, $K$, of 0.1 . Using a surface buoyancy flux, $B$, of $3 \times 10^{-7}$, which corresponds to a heat flux of approximately $60 \mathrm{~W} / \mathrm{m}^{2}$, and solving iteratively for $\mathrm{d}$ as a function of time gives the analytic predicted convective depth shown by the solid line in figure 4.6. For comparison, the predicted depth from equation 4.1 for constant stratification, using $N=1.2 \times 10^{-6} \mathrm{~s}^{-1}$ (the average value over the top 500 meters of the exponential profile) is shown as a dotted line.

Evidently, the initial convection is fairly well represented using an approximate vertical average of the vertically varying stratification. This can be seen by looking at the limit of equation 4.6 when $d \ll \sigma$. Taylor expansion forms for the exponentials given by:

$$
e^{d / \sigma} \approx 1+\frac{d}{\sigma}+\cdots
$$

can be substituted into equation 4.6 to give:

$$
B T=\frac{g}{\rho_{0}}\left(\frac{K d^{2}}{\sigma}\right) .
$$

Solving for $\mathrm{d}$ then gives:

$$
d=\sqrt{\frac{B T}{\frac{g K}{\rho \sigma}}}
$$

The vertical derivative of the original stratification is simply:

$$
\frac{\partial \rho}{\partial z}=-\frac{K}{\sigma} e^{z / \sigma}
$$


Applying the same Taylor series expansion, for $z \ll \sigma$, and keeping only the first term, gives:

$$
\frac{\partial \rho}{\partial z} \approx-\frac{K}{\sigma} .
$$

Or, in terms of the buoyancy frequency,

$$
N^{2} \approx \frac{g}{\rho} \frac{K}{\sigma}
$$

Substitution of this approximate buoyancy forcing into equation 4.9 reproduces the linear result:

$$
d \approx \sqrt{\frac{B T}{N^{2}}}
$$

Thus, in the limit of very shallow convection, the deepening is well approximated by the constant stratification expression. However, once the convection starts to penetrate into the region of significantly lower stratification which lies more than one e-folding scale from the surface, the difference between the prediction assuming constant $N^{2}$, and that using an exponential $N^{2}$ formula, becomes large. The dramatic increase in rate of convective deepening once the pycnocline has been ventilated is the readily apparent reason for this difference.

In order to compare with these analytical curves, I have run the SPEM in an extremely simple experiment. I configured the model as a square box with uniform surface cooling. The parameters associated with this run is listed as run N1 in table 4.1. There is no mean flow, and the now unnecessary sponge layers and radiative boundary conditions have been removed. Furthermore, the model is initialized with an exponential stratification identical to that used for the analytic calculation. As expected, the mixed-layer in this run deepens uniformly everywhere in the domain. Values for the penetration depth at five day intervals, as diagnosed from the model run, are shown as open circles in figure 4.6. Comparison with the implicit analytical result is extremely good. 


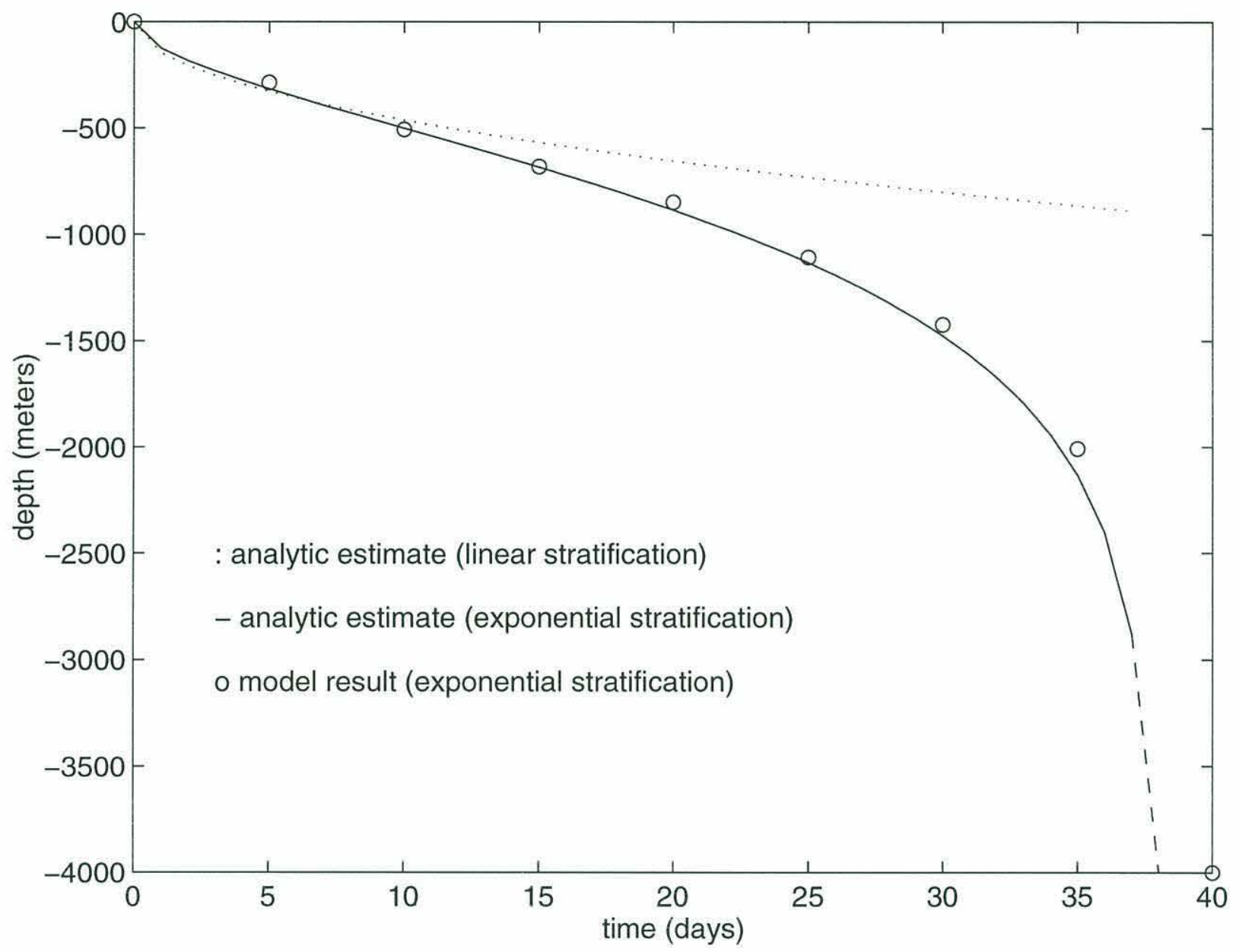

Figure 4.6: The solid line shows the expected penetration depth as a function of time using the implicit formula. The surface buoyancy flux is $1.5 \times 10^{-7}$ and the exponential stratification has an e-folding scale of 500 meters. The open circles show the result of a primitive-equation run with the same parameters, which is in good agreement with the analytic prediction. Note that the model data, because it has been saved only at 5 day intervals, first shows convection reaching the 4000 meter bottom of the domain on day 40 , but this is not inconsistent with the analytic curve. For purposes of comparison, the dotted line shows the analytic prediction using assuming a uniform stratification with $N / f=11$, which is the average value over the top 500 meters of the actual profile. It is evident that the initial penetration is close to the square root dependence expected for constant stratification, but that as the less stratified waters begin to ventilate, the rate of convective deepening is significantly enhanced. 


\begin{tabular}{|c|c|c|c|c|c|}
\hline Run & $\begin{array}{c}\text { Fractional } \\
\text { Height }(\delta)\end{array}$ & $\begin{array}{c}\text { Aspect Ratio } \\
(\Delta)\end{array}$ & $\begin{array}{c}\text { Rossby Number } \\
(R)\end{array}$ & $\begin{array}{c}\text { Stratification } \\
\text { Parameters }(\sigma, K)\end{array}$ & $\begin{array}{c}\text { convective Rossby } \\
\text { Number }\left(R_{N}\right)\end{array}$ \\
\hline N1 & 0 & 0 & 0 & $500,0.1$ & $9.7 \times 10^{-2}$ \\
\hline N2 & 0.5 & 0.16 & 0.08 & $500,0.1$ & $9.7 \times 10^{-2}$ \\
\hline
\end{tabular}

Table 4.1: A list of parameters associated with each SPEM run incorporating exponential backgound stratification.

\subsection{A Simple Isopycnal Doming Experiment}

This section describes a simple use of equation 4.6 to demonstrate doming isopycnals as a mechanism for convective preconditioning. A hypothetical section through a region of domed isopycnals is shown in figure 4.7. This section is comprised of 32 separate locations, each with an exponential density profile given by equation 4.2 . The doming is produced invoking the squeezing paradigm appropriate for flow over topography by varying the e-folding scale from a value of 400 meters at the edges to 100 meters in the center. For reference, the central profile is identical to the 100 meter e-folding profile shown in figure 4.3. The surface and deep water densities are the same throughout the section.

Given this set of exponential profiles, iterative solutions to equation 4.6 can be used to solve for the mixed-layer penetration depth as a function of position within the section. The surface density flux used for this calculation is equivalent to $30 \mathrm{~W} / \mathrm{M}^{2}$. Figure 4.8 shows the results as a time series of mixed-layer depths at two day intervals. For the first ten days the mixed-layer deepens throughout the domain, with slightly deeper penetration in regions with the lowest $N^{2}$ values, away from the central doming. However, the pycnocline is first penetrated in the domed region, leading to rapid penetration of a convective chimney beginning just after day 10 and reaching to the bottom of the domain by day 16. This convective deepening of a chimney in the region of isopycnal doming is a direct consequence of the fact that the initial density is not a constant $N^{2}$ profile. 


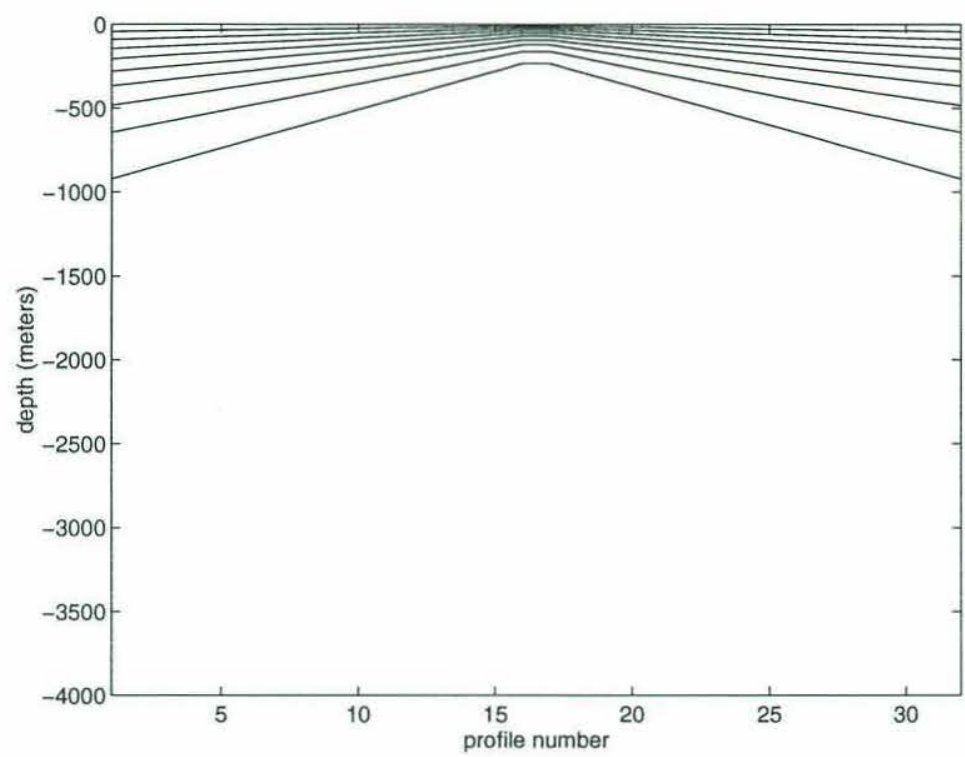

Figure 4.7: This figure shows a section through a hypothetical region of domed isopycnals. Each profile along the section has an exponential density profile. The e-folding scale of the exponential increases linearly from a value of 400 meters at the edges to 100 meters in the center. The surface temperature and deep water temperatures are held constant.

\subsection{Topographic Preconditioning Experiments}

Given the important role that nonuniform background stratification can potentially play in terms of the effectiveness of domed isopycnals as a source of preconditioning, it is interesting to investigate the effect such a stratification will have in the specific topographic preconditioning scenario associated with flow over isolated topography. The first question that needs to be addressed in this context is to what extent the initial Taylor cap spinup process and steady-state flow field before the onset of surface cooling are affected by an exponential background stratification. For this purpose, I have run the SPEM model with the parameter settings listed in table 4.1 as run N2. This run is configured identically to the constant stratification spinup runs, S1 and S2 listed in Table 3.2, with the exception of the background stratification, which is chosen to be the exponential profile used in run N1.

Figure 4.9 shows the density field on slices through the center of the domain for the initial condition and the spun-up steady-state flow. The doming of isopycnals associated 

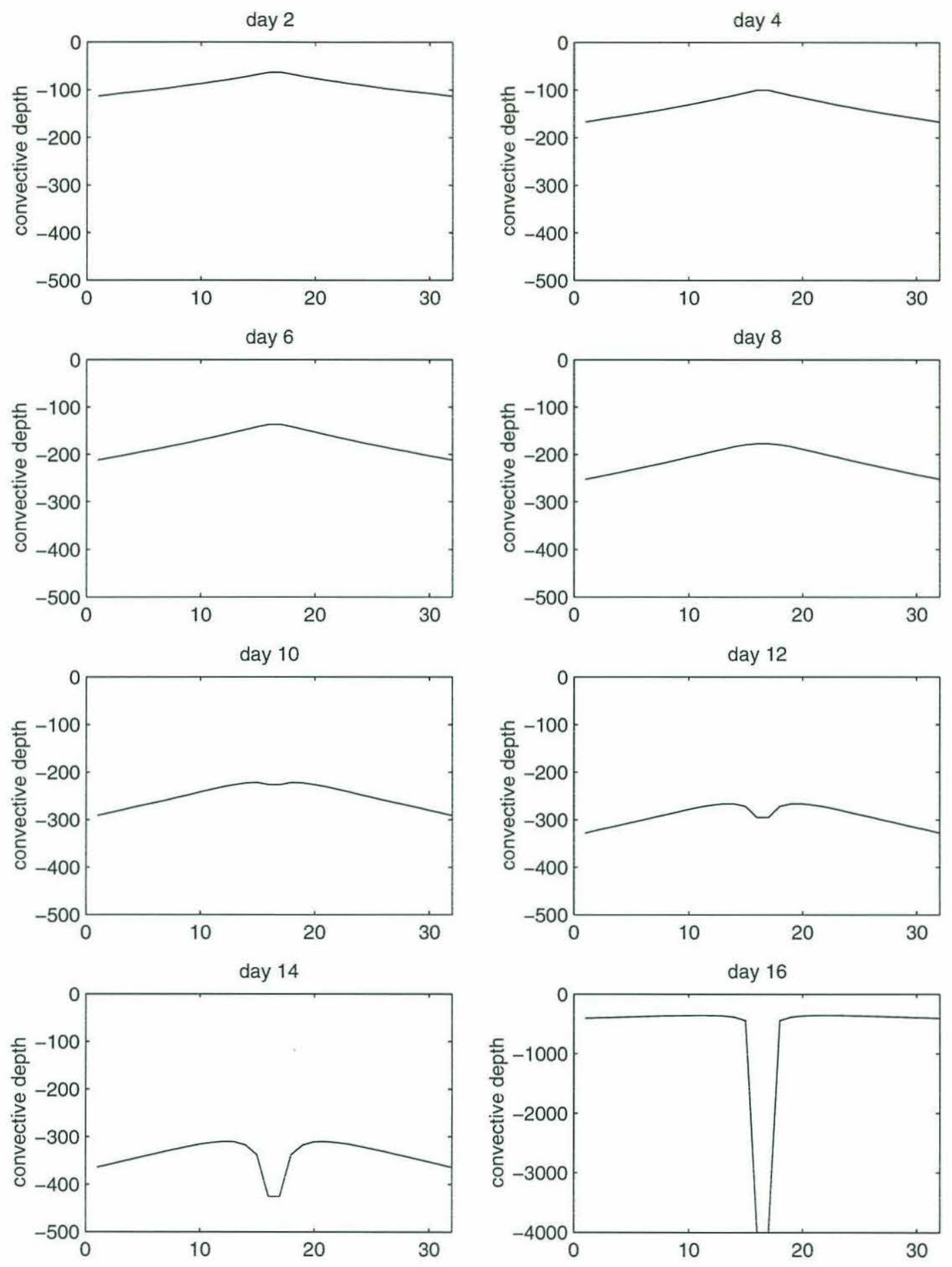

Figure 4.8: A time history of the mixed-layer depth associated with uniform surface cooling over the hypothetical section through a region of domed isopycnals as predicted by the analytic solution to convection into an exponential density profile. 

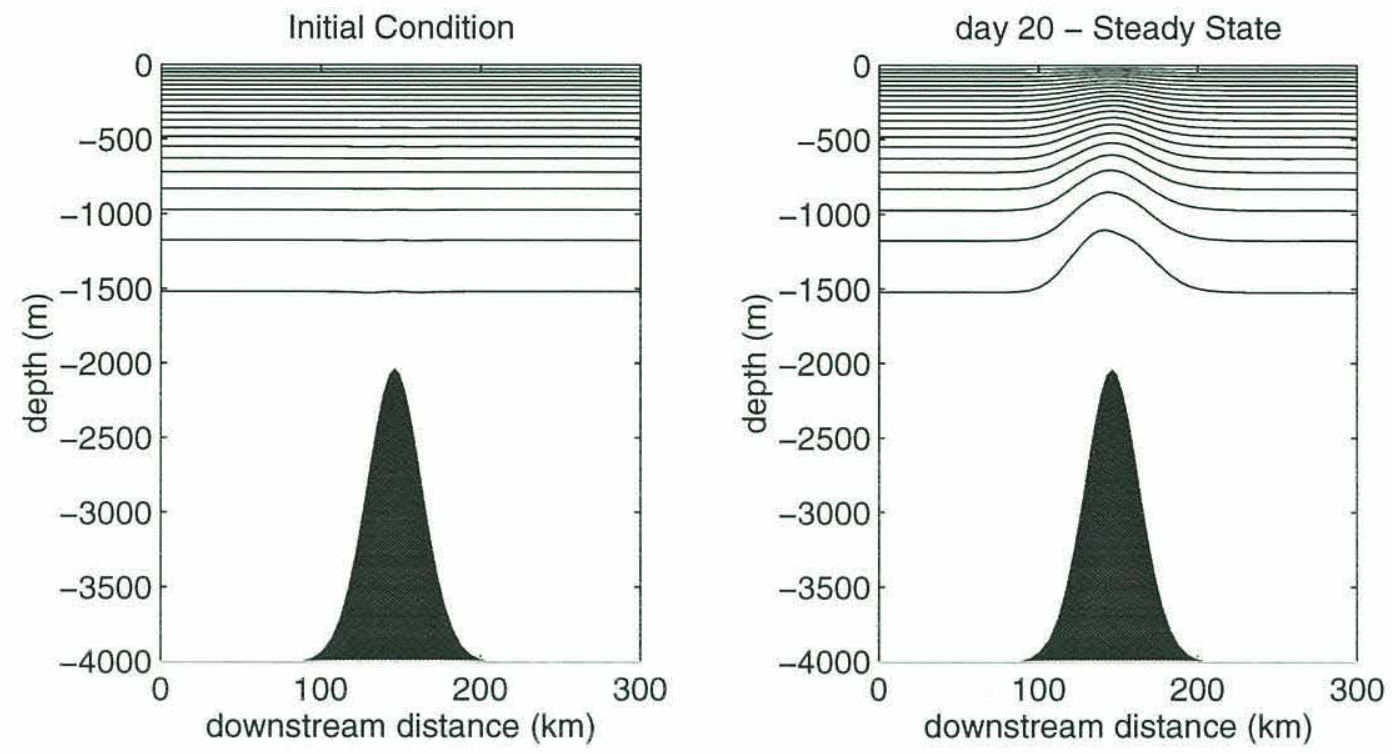

Figure 4.9: Slice plots of the density field through the center of the domain of run N2. On the left is the initial condition and on the right the spun up steady-state at day 20 .

with the spun-up anticyclonic Taylor cap is evident. It is interesting to note that despite the doming of the pycnocline, none of the isopycnals intersect the surface, indicating that the doming is produced by squeezing of isopycnals, rather than lifting. This is not due to a constant temperature boundary condition; recall from Chapter 2 that a zero gradient condition, associated with zero heat flux, is employed at the upper boundary. In fact, it is because there is no explicit vertical mixing in the interior that it is impossible for isopycnals to outcrop without surface forcing having been applied.

A time series of horizontal plots of the density field at 500 meters depth in figure 4.10 shows the spinup process. This figure can be compared with figure 2.3 , which shows the same time series, albeit at 2000 meters depth, for run S2. Run S2 is spun-up with the same parameters as this run, N2, with the exception of the background stratification, which is constant with an equivalent top-to-bottom density difference as the exponential used in run N2. Comparing the two figures, it is apparent that the nonuniformity of the background stratification has little effect on the nature of the spinup process. However, the details of the steady-state flow, especially the vertical extent of the region of trapped 
flow, which depend critically on the stratification, are expected to differ. The analytic WKB approximation for a steady-state Taylor cap in an exponentially stratified fluid is presented by Owens and Hogg [1980]. Their results, indicating a taller cap for an exponential stratification relative to one with a weak linear stratification equivalent to the deep part of the exponential, are consistant with the numerical results in this chapter.

In order to look at differences in the steady-state flow, a passive tracer slice through the center of the domain is shown in figure 4.11. This figure should be compared with the equivalent tracer slice for run $\mathrm{S} 2$, shown in figure 3.7. The most obvious difference between these two figures is that the region of trapped fluid, demarcated by the zero contour, extends significantly higher in the water column, in fact all the way to the surface, in run N2. Although the top to bottom total density differences are the same in these two runs, the exponential stratification run has very low values of buoyancy frequency at depth, allowing the influence of the topography to penetrate much higher into the water column.

Another interesting phenomenon visible in figure 4.11 , is the fact that the tilting over of the Taylor cap with height is predominantly occurring near the surface. With constant background stratification the Taylor cap also tends to tilt to the left (looking upstream) with height above the topography, but the tilting is more or less constant with height. One way of thinking about this tilting with height is in the context of a layer model. The lowest layer will have a region of trapped flow on the right side of the underlying topography (looking downstream). The next layer up feels this stagnant region in the same manner as it would topography and thus its own trapped retion is slightly to the right of the trapped region in the layer below. For stonger stratification the layers are compressed, leading to a stronger tilting with height. Thus, this tendency of the cap to tilt is directly related to the magnitude of the stratification, and a surface-intensified exponential stratification leads to a surface intensification of the tilting.

Surface cooling equivalent to approximately $60 \mathrm{~W} / \mathrm{m}^{2}$ is imposed uniformly over the surface of the model domain once the steady-state has been reached at day 20. A time 

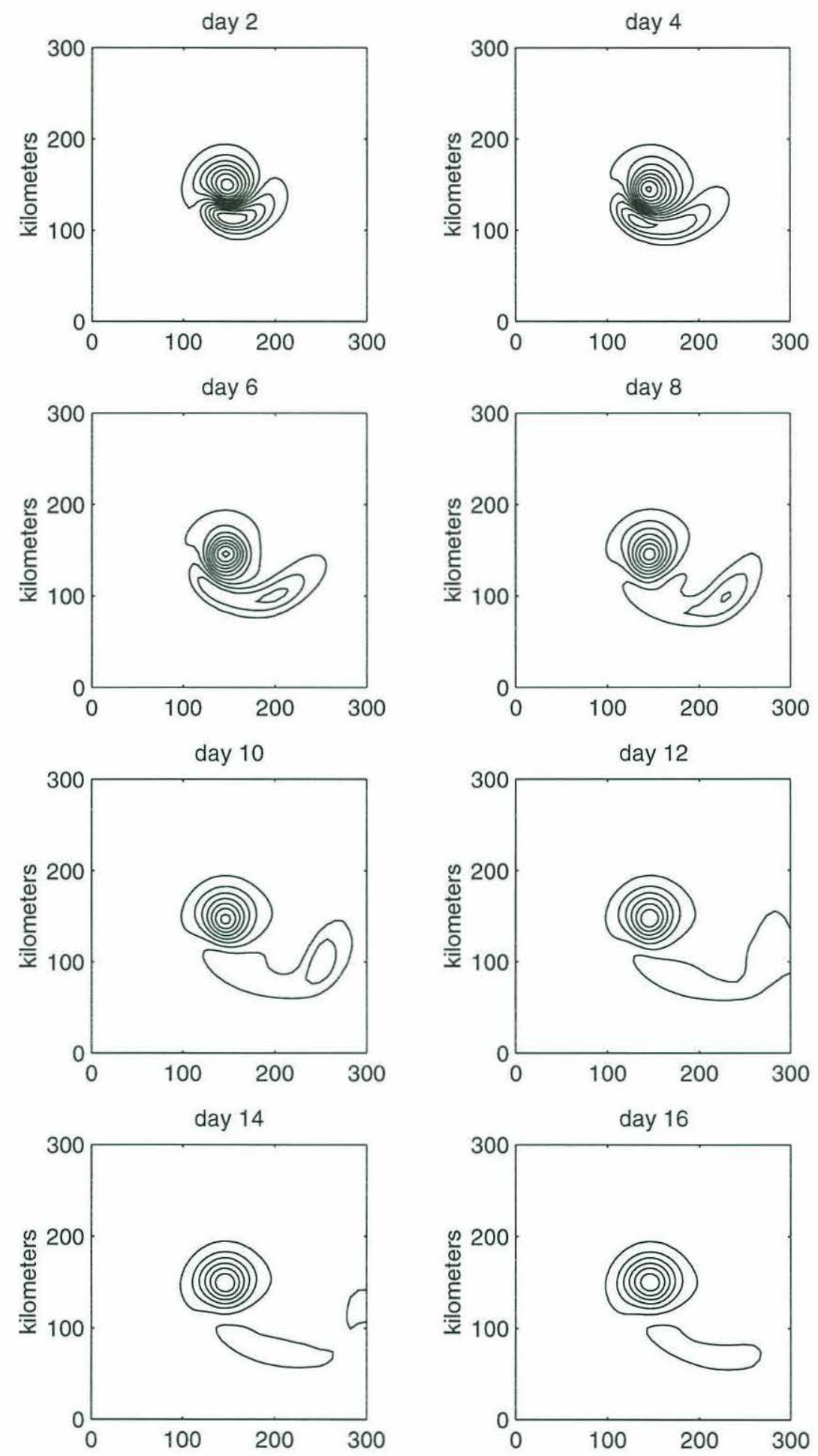

Figure 4.10: Time series of slab plots of the density field at 500 meters depth for the spinup of run N2, with exponential background stratification. 


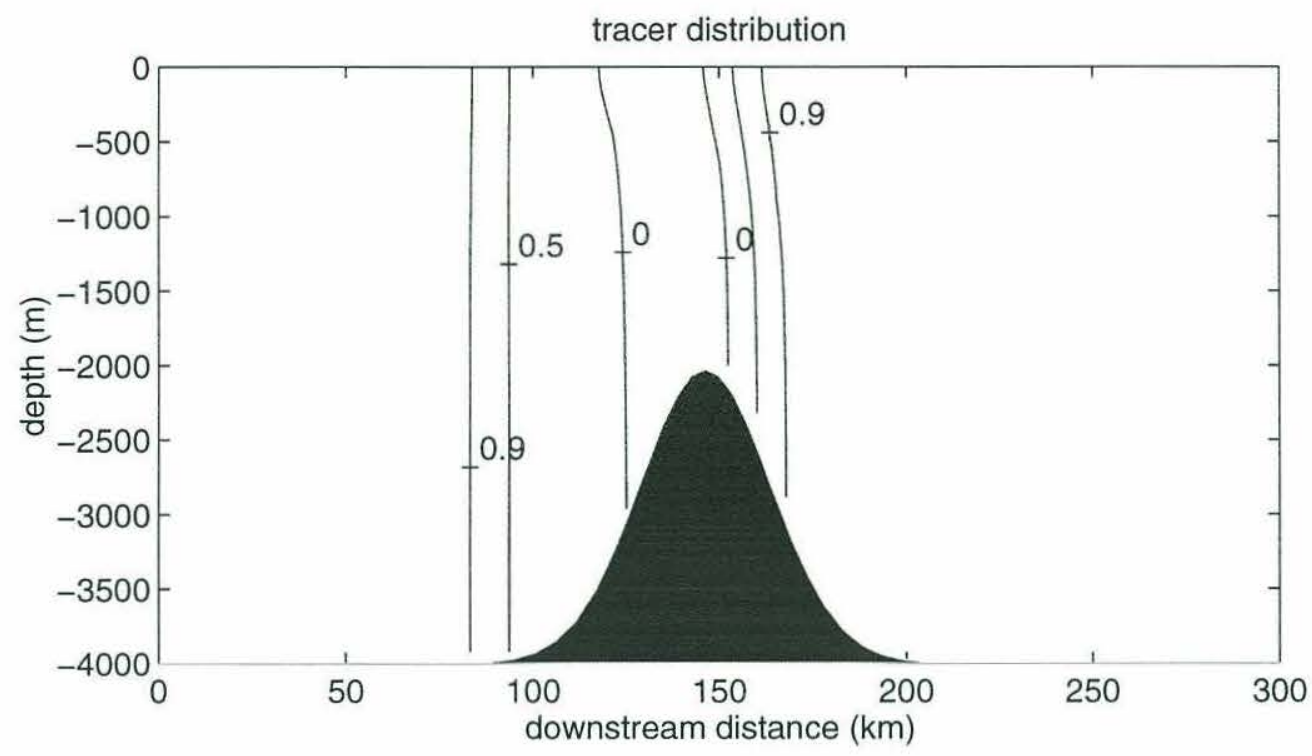

Figure 4.11: A slice plot of passive tracer distribution through the center of the steady-state of run N2. Tracer values of zero indicate water which remains from the initial state. Values of one have been ventilated from upstream.

history of density slice plots through the center of the domain at 20 day intervals is shown in figure 4.12. After 20 days of cooling, at day 40 of run time, a convective chimney has begun to form over the flank of the seamount, as in the constant stratification runs. For comparison with a constant stratification run with identical surface cooling and top to bottom total density gradient, recall figure 3.12 which shows a similar time series of density slices for run C1. After only 20 days of cooling the two runs have evolved similarly; both have a convective chimney reaching to approximately 1000 meters depth trapped over the flank of the seamount, with a somewhat less deep ambient mixed-layer in the rest of the domain. However, after another 20 days of cooling the two runs show a marked difference. In run N2, the pycnocline has fully outcropped at this point, leading to surface ventilation of the densest water in the domain. The convective chimney soon reaches to the bottom and contains the densest water found in the domain. A new dense water mass is being formed in this case. In the constant stratification run, $\mathrm{C} 1$, however, because the deep water is still as strongly stratified as the surface layers, the penetration depth of the chimney is not nearly as dramatic, and the densest bottom waters remain 
day 40

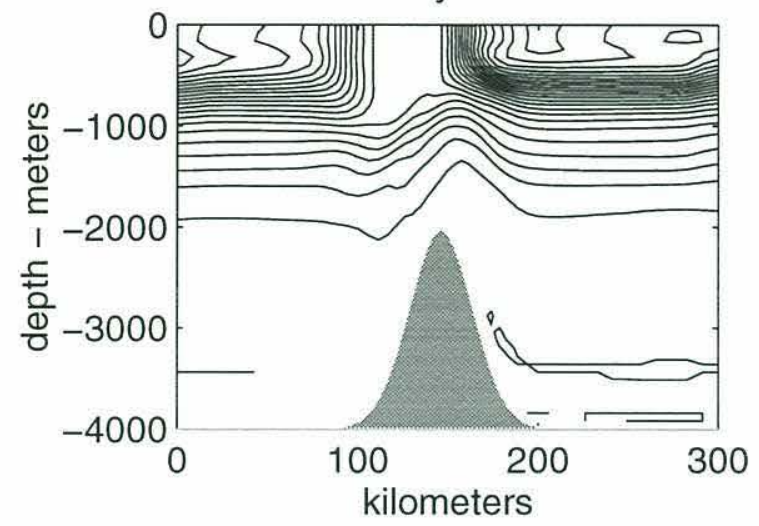

day 80

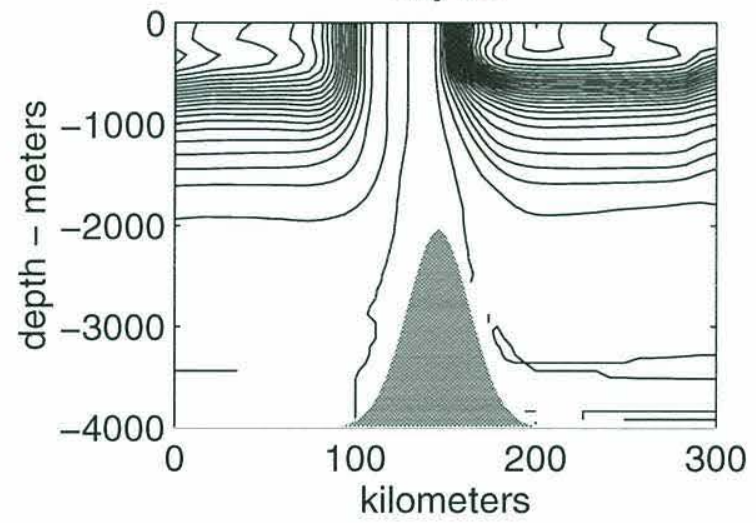

day 60

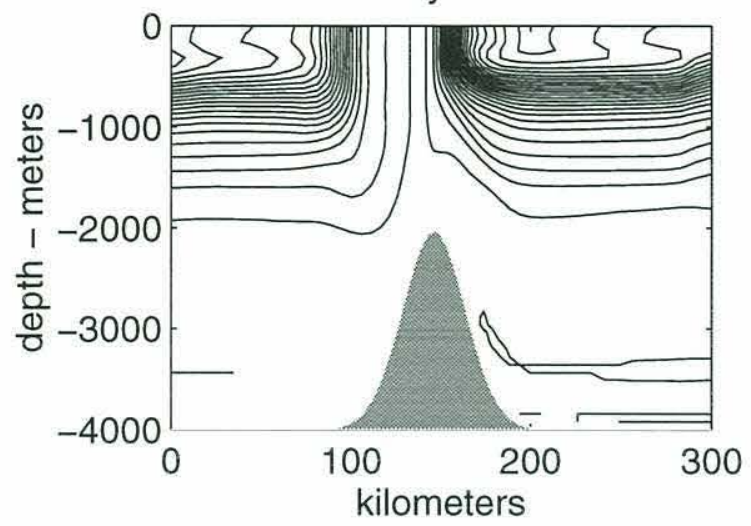

day 100

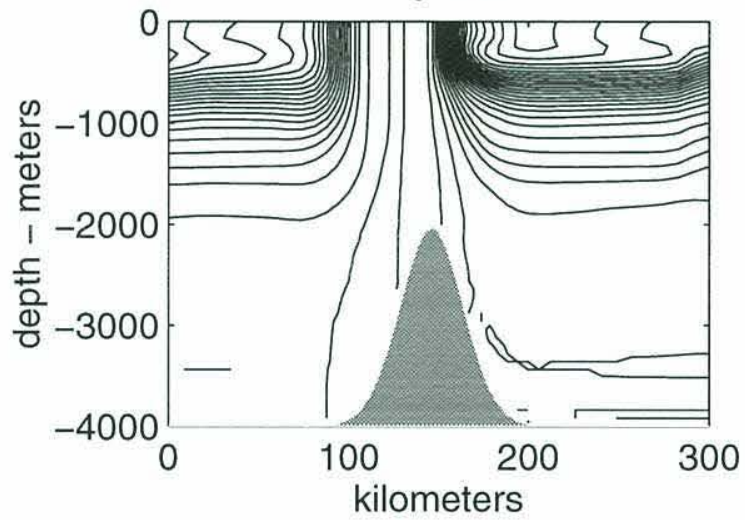

Figure 4.12: A time series of slices through the center of the domain from run N2 showing the convective penetration localized over the seamount. One key difference, as compared to constant stratification runs is the enhanced deepening leading to the generation of the densest water found anywhere in the domain once the pycnocline has been ventilated.

unventilated.

\subsection{Conclusions}

A simple exponential stratification is used to examine the effect of variable background stratification on preconditioning of convection. A one-dimensional solution for the depth of convection into an exponential background stratification for a given surface density flux is found. The one-dimensional solution is used to examine how a region of domed 
isopycnals will convect given uniform surface cooling. The initial penetration depth is slightly deeper away from the doming. The deep, less stratified, region is first ventilated in the center of the doming region, however, because of the proximity of the pycnocline to the surface. Once the pycnocline has outcropped, a rapid deepening of a deep convective chimney occurs.

The SPEM model, configured with an exponential background stratification and uniform surface cooling, but without bottom topography or mean flow, gives a rate of convective deepening in excellent agreement with the one-dimensional analytical result. In order to investigate the effect of nonuniform stratification in the topographic preconditioning problem, a Taylor cap spinup is performed with an exponential background stratification. The spinup process is qualitatively similar to the constant stratification spinup runs in Chapter 3. A cyclone and anticyclone are spun up over the topography, the cyclone is soon advected downstream, exiting the domain, while the anticyclone remains topographically trapped. The region of trapped flow extends higher in the water column than for runs with the same top-to-bottom density difference but a uniform background stratification, due to the low stratification at depth.

Uniform surface cooling leads, initially, to a convective chimney centered over the seamount flank as in uniform stratification runs. Once the pycnocline has outcropped however, the chimney deepens to the bottom of the domain rapidly, as in the doming experiment with the analytical, one-dimensional model. Thus, given a slightly more realistic stratification, with a near surface intensification in buoyancy frequency, the surface outcropping of sub-pycnocline isopycnals becomes the primary factor leading to convective penetration into the deep water. However, mean flow topographic interaction still serves to precondition the region where the pycnocline first outcrops, due to the domed isopycnals associated with the anticyclonic flow over the seamount. 


\section{Chapter 5}

\section{Shutdown of Convective Deepening}

\subsection{Introduction}

In the previous chapters, this thesis has been primarily concerned with the preconditioning phase of open ocean convection. Although the violent mixing phase is also included in the numerical integrations, nonhydrostatic physics at the kilometer and smaller scales, which describes the mixing process, is parameterized with the use of a simple convective adjustment scheme. The final phase of the convective process, sinking and spreading of the dense chimney, is examined in this chapter. In particular, details of the shutdown of convective deepening of a topographically preconditioned chimney are compared and contrasted to the more familiar shutdown of chimneys which are generated beneath isolated regions of strong surface cooling.

Generally, the separation of convection into a mixing phase followed by a spreading phase is predicated on the simplified picture of two separate dynamical regimes. During the mixing phase, it is assumed that the surface flux is balanced by vertical entrainment of heat from below as the chimney burrows into deeper water. In contrast, during the spreading phase, continued surface buoyancy losses are balanced by horizontal fluxes as the chimney breaks up and exchanges heat with the surrounding stratified water. This horizontal exchange is usually thought to occur as a result of baroclinic instability of the 
chimney, which leads to substantial horizontal eddy fluxes. Although this eddy exchange process is certainly an important one, in the context of topographically preconditioned chimneys it is also possible for the horizontal fluxes to be accomplished by the mean background flow.

\subsection{Mechanisms}

In general, convective chimneys in the ocean do not penetrate all the way to the ocean floor. There are several reasons why this may be the case. In the context of a newly formed, dense, surface water mass sinking into a stratified fluid, one might expect the dense water to sink until it reaches a depth where it matches the ambient density and is neutrally buoyant. On the other hand, if convection is forced by a continuous surface flux, rather than a specific surface density, the chimney might be expected to stop deepening when it comes under rotational control, and is broken up by strong baroclinic instability. The breakup of a convecting chimney due to baroclinic instability is examined in detail

by Visbeck et. al. [in press]. Applying closure ideas of Green [1970] and Stone [1972] to parameterize the horizontal transfer of heat by baroclinic eddies in terms of mean flow quantities, they deduce scaling laws for the maximum depth of convection and the time required to reach that depth:

$$
\begin{aligned}
h_{f} & =\gamma \frac{(B r)^{1 / 3}}{N}, \\
t_{f} & =\beta\left(\frac{r^{2}}{B}\right)^{1 / 3} .
\end{aligned}
$$

Here $r$ is the chimney radius, $B$ the suface buoyancy flux and $N$ the buoyancy frequency of the background stratification. $\gamma$ and $\beta$ are constants of proportionality with values of $3.9 \pm 0.9$ and $12 \pm 3$ respectively. These constants are derived from numerical integrations as well as laboratory experiments. 


\begin{tabular}{|l|c|c|c|c|c|}
\hline Run & $\begin{array}{c}\text { Fractional } \\
\text { Height }(\delta)\end{array}$ & $\begin{array}{c}\text { Rossby Number } \\
(R)\end{array}$ & $\begin{array}{c}\text { Radius of cooling disk, or } \\
\text { Efolding scale of seamount }\end{array}$ & $\begin{array}{c}\text { convective Rossby } \\
\text { Number }\left(R_{N}\right)\end{array}$ & $\begin{array}{c}\text { Stratification } \\
(N / f)\end{array}$ \\
\hline D1 & 0.5 & 0 & $20 \mathrm{~km}$ & $9.7 \times 10^{-2}$ & 5.556 \\
\hline C4 & 0.5 & 0.08 & $25 \mathrm{~km}$ & $9.7 \times 10^{-2}$ & 5.556 \\
\hline C6 & 0.5 & 0.08 & $25 \mathrm{~km}$ & $9.7 x 10^{-2}$ & 3.0 \\
\hline
\end{tabular}

Table 5.1: External parameters for convective shutdown experiments.

In order to compare the dynamics of a chimney generated in a quiescent fluid beneath a localized cooling patch as in Visbeck et. al. with topographically preconditioned chimneys, a disk-cooling experiment was performed with the SPEM model. The model friction parameters, timestep, and resolution are kept identical to the topographic run, C4, with which the comparison is to be made. This disk-cooling experiment is referred to as run D1 henceforth. Two topographically preconditioned runs are presented for comparison. The first is run C4, which is discussed in Chapter 3. The second, run C6, is identical to $\mathrm{C} 4$ in all respects except that a lower background stratification is used. All three runs, and the relevant non-dimensional parameters, are listed in Table 5.1. In each run the convective deepening is shut down in a different manner.

\subsubsection{Disk-Cooling Experiment - D1}

Figure 5.1 shows a vertical slice of the density field through the disk-cooled chimney in run D1. Note that in this run the bottom topography plays no role since there is no mean flow. As expected given the results from Visbeck et. al., the chimney first deepens following the analytic one-dimensional prediction. The strong horizontal density gradients separating the chimney from its surroundings imply an associated vertical shear in the velocity field. This shear manifests itself as a strong cyclonic rim current at the surface and a counter-rotating anticyclonic current at depth. Figure 5.2 shows horizontal slices through the domain at 1500 meters depth, near the base of the convective chimney. After about 20 days of cooling, strong baroclinic instability of the rim current leads 


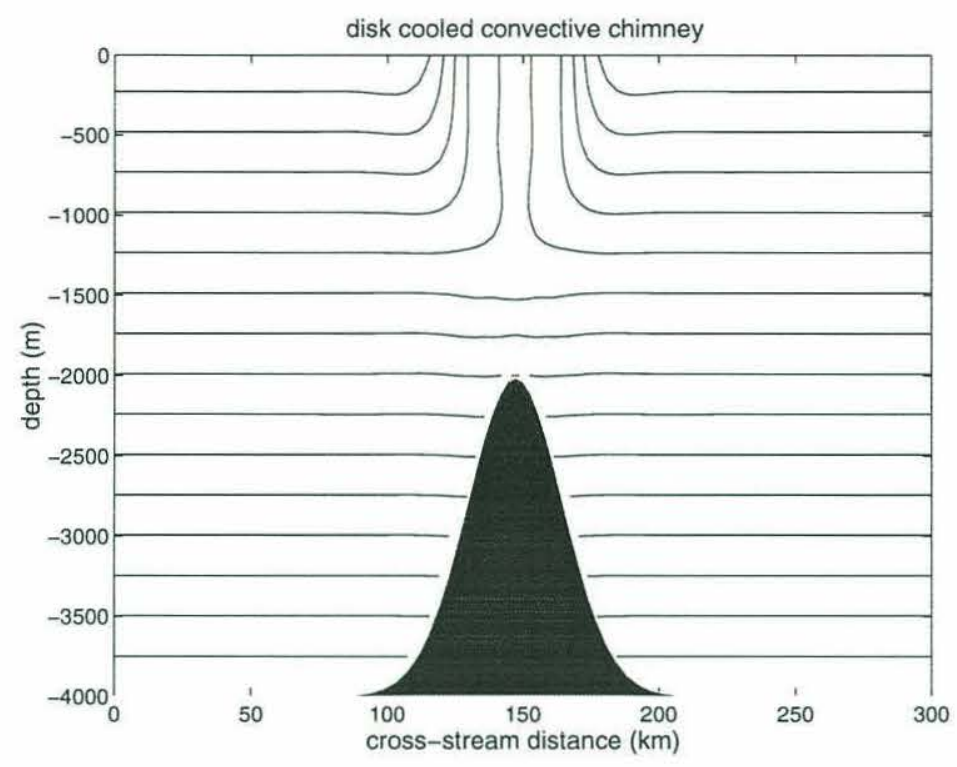

Figure 5.1: A cross section of the density field in run D1 after 20 days of cooling. In this case the bottom topography is not dynamically active since there is no mean flow. The chimney is being generated by a disk shaped region of cooling at the surface with a horizontal radius of 20 kilometers.

to large enough horizontal fluxes to shut down the convective process. The baroclinic instability is, in this case, visible as a mode four disturbance growing on the initially circular rim current. This instability of the rim current associated with chimneys formed beneath disk-shaped cooling regions is described in detail by several authors, including Jones and Marshall [1993], Hermann and Owens [1993], and Visbeck et.al. [in press].

The convective depth as a function of time from this model run is shown in figure 5.3 along with the one-dimensional, non-penetrative convective limit. Around day 20 the model chimney deepening starts to shut down. This departure from the one-dimensional analytic limit is due to the horizontal fluxe of heat as the baroclinic eddies carry stratified water underneath the cooling patch. This balance between horizontal advection of heat by baroclinic eddies and the surface cooling is precisely the paradigm proposed by Visbeck et. al. In Table 5.2, the final depth reached and the time required to achieve this depth for the chimney from run D1 are compared with values determined using equations 5.1 and 5.2. Although the depth reached here is on the high side of the range predicted, 

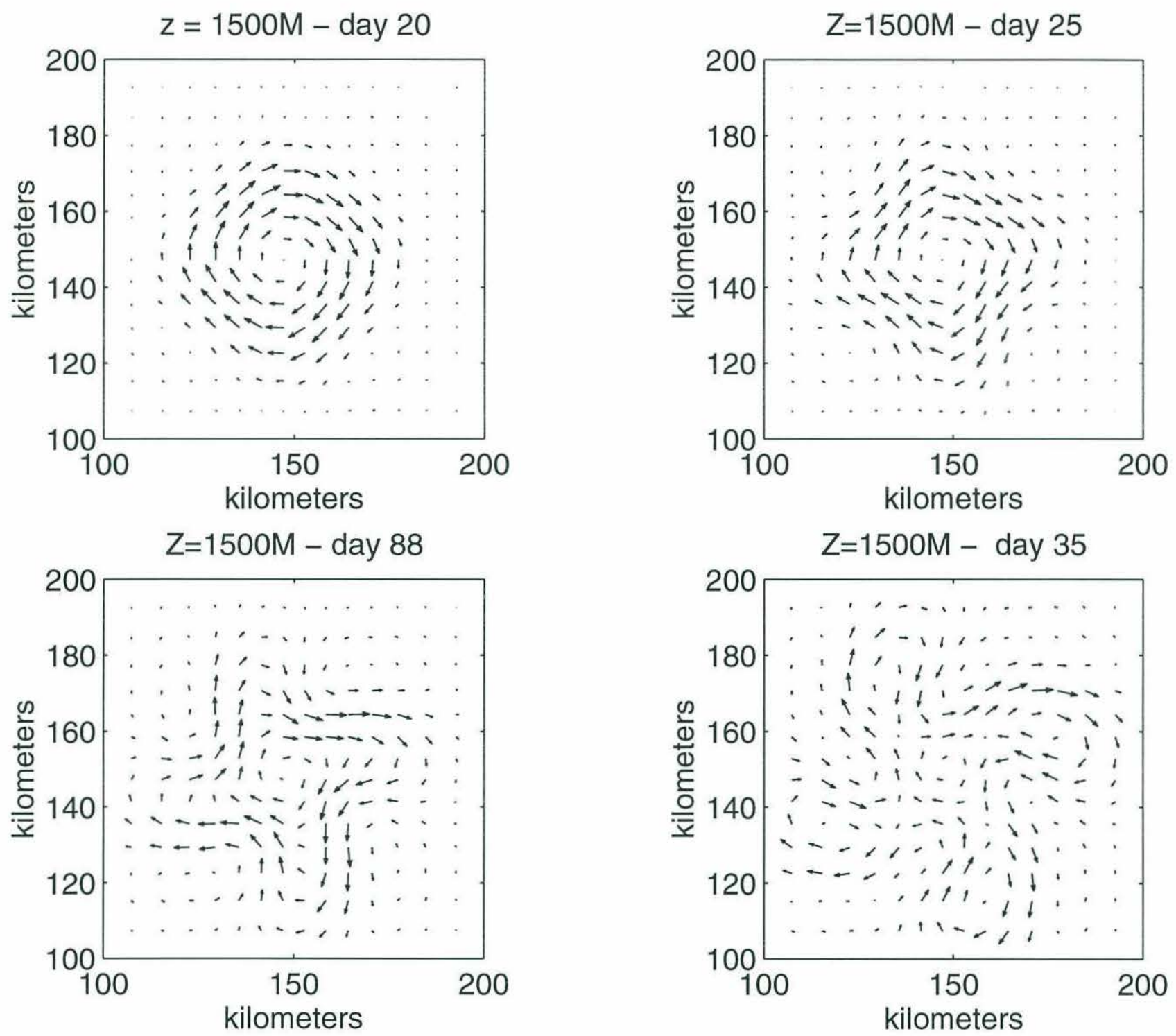

Figure 5.2: A time series showing the evolution of the horizontal velocity field at 1500 meters depth, near the base of the disk-cooled chimney in run D1. After initially deepening for twenty days the rim current begins to show a growing mode four instability. Over the next 15 days this instability grows rapidly. 


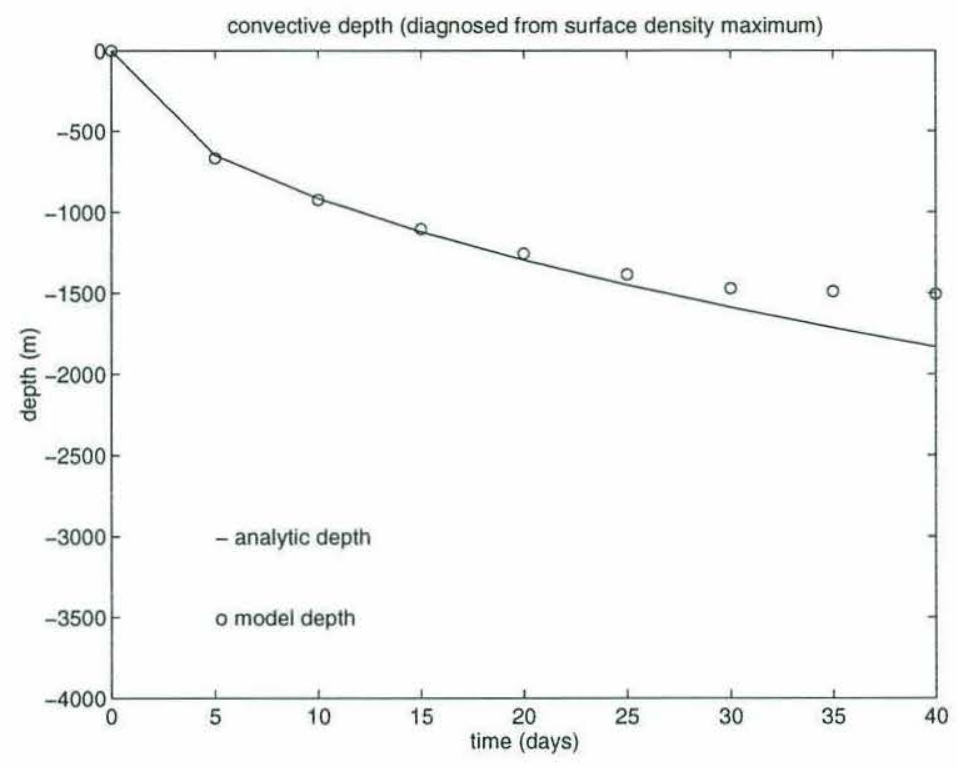

Figure 5.3: The time history of convective deepening of the disk-cooled chimney in run D1 is plotted with circles. The solid line shows the one-dimensional, non-penetrative convection limit for comparison. After roughly 25 days of cooling the horizontal fluxes of heat associated with baroclinic instability of the chimney have become sufficient to shut down the convection.

the agreement is fairly good. It is rewarding to see the similarity of these results despite the very different treatment of the convective process in the two studies. In the Visbeck study, the numerical runs were done with a nonhydrostatic model resolving plume scales, whereas the current model uses a simple convective adjustment scheme to parameterize the mixing process. Both numerical representations of convection, in addition to being mutually consistent, are also consistent with the laboratory results obtained by Hufford [1994] (which are also used in the Visbeck et. al. study).

\subsubsection{Stable Topographic Preconditioning Experiment - C4}

The convective depth time series for run $\mathrm{C} 4$ is shown in figure 5.4. Recall that for the topographic runs the cooling commences after an initial 20 day spinup period required to generate a steady-state Taylor cap over the topography. Although the convective depth history appears to be quite similar to that for the disk-cooling run (D1), in this case baroclinic eddies do not play a role in shutting down the convective deepening. After 


\begin{tabular}{|l|c|c|c|}
\hline & Visbeck et.al. Formula & Disk-Cooling & Topographic Preconditioning \\
\hline $\begin{array}{l}\text { Depth of convection (meters) } \\
\text { Time to reach } \\
\text { maximum depth (days) }\end{array}$ & $1000 \pm 200$ & 1250 & 1500 \\
\hline
\end{tabular}

Table 5.2: A comparison of the maximum depth of convection and the time taken to reach that steady-state depth for a chimney formed by cooling over a disk shaped region and one formed by uniform cooling over as topographically preconditioned domain.

50 days of cooling the model has come to a steady-state with no sign of instability of the chimney. Despite continued surface forcing, the convective deepening has been shut down. In this case, the surface cooling is being balanced by horizontal fluxes of heat due to the mean flow crossing isopycnals.

Figure 5.5 shows a series of constant depth slices of the density and velocity fields near the seamount for this steady-state. Away from the seamount, the mixed-layer density increases with distance downstream as discussed in section 3.2. There is a pronounced density anomaly, associated with the deep convecting chimney, centered over the right flank of the topography in the region of recirculating flow. In addition, there is a dense tail of enhanced mixed-layer deepening extending downstream from the seamount. Deeper down, at the 1500 meters depth, the isopycnal signal of the Taylor cap itself is visible, slightly to the right, looking downstream, of the base of the convective chimney. At 2000 meters depth, below the steady-state penetration depth of the chimney, only the signal of domed isopycnals associated with the Taylor cap is visible. Although the horizontal transport of heat by the mean flow across density gradients can explain the shutdown of convection, it does not explain why the chimney is not susceptible to growing baroclinic eddies. The strong horizontal density gradients around the chimney, in the presence of zonal flow, as visible for example in the surface and 1000 meter depth slices, are one indication of the likely occurrence of baroclinic instability, yet the chimney remains stable. 


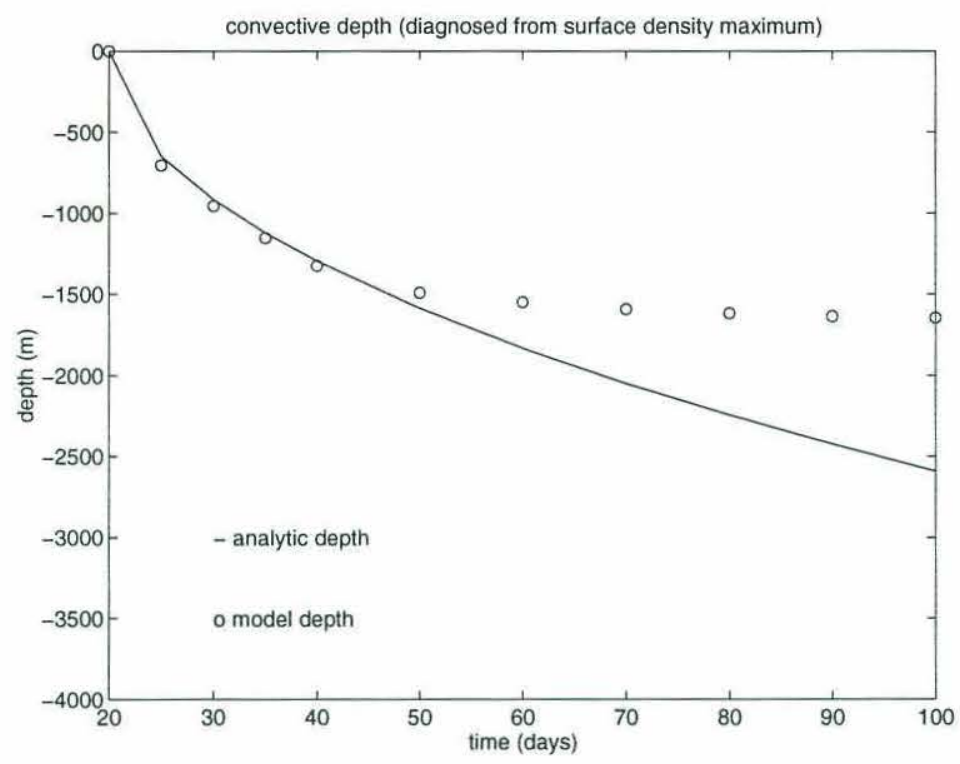

Figure 5.4: The time history of convective deepening of the topographically preconditioned chimney in run $\mathrm{C} 4$ is plotted with circles. The solid line shows the one-dimensional, nonpenetrative convection limit for comparison. After roughly 30 days of cooling the horizontal fluxes of heat associated with mean flow across isopycnals above the region of fluid trapping has become sufficient to shut down the convection.

\subsubsection{Unstable Topographic Preconditioning Experiment - C6}

Run C6 is different from run C4 only in that the background stratification is lower; the buoyancy frequency, $N$, is $3 \times 10^{-4} s^{-1}$ instead of $5.6 \times 10^{-4} s^{-4}$. This change allows significantly deeper penetration of the convective chimney. As shown in figure 5.6, the chimney deepens as predicted by the one-dimensional, non-penetrative estimate until it hits the bottom of the domain, which is denoted by the dashed line at 4000 meters depth. Shortly after reaching the bottom, however, the chimney sheds a baroclinic eddy. Unlike the robust mode four instability of experiment D1, however, the instability is confined to the region of enhanced deepening in the wake of the seamount.

Figure 5.7 shows a time history of the initial eddy shedding event which leads to the sudden shut-off in convective deepening seen in figure 5.6 at day 90. Clearly, based on a comparison of runs $\mathrm{C} 4$ and $\mathrm{C} 6$, the stability of topographically preconditioned chimneys is dependent on the exact parameter regime in which the chimney forms. Although a 

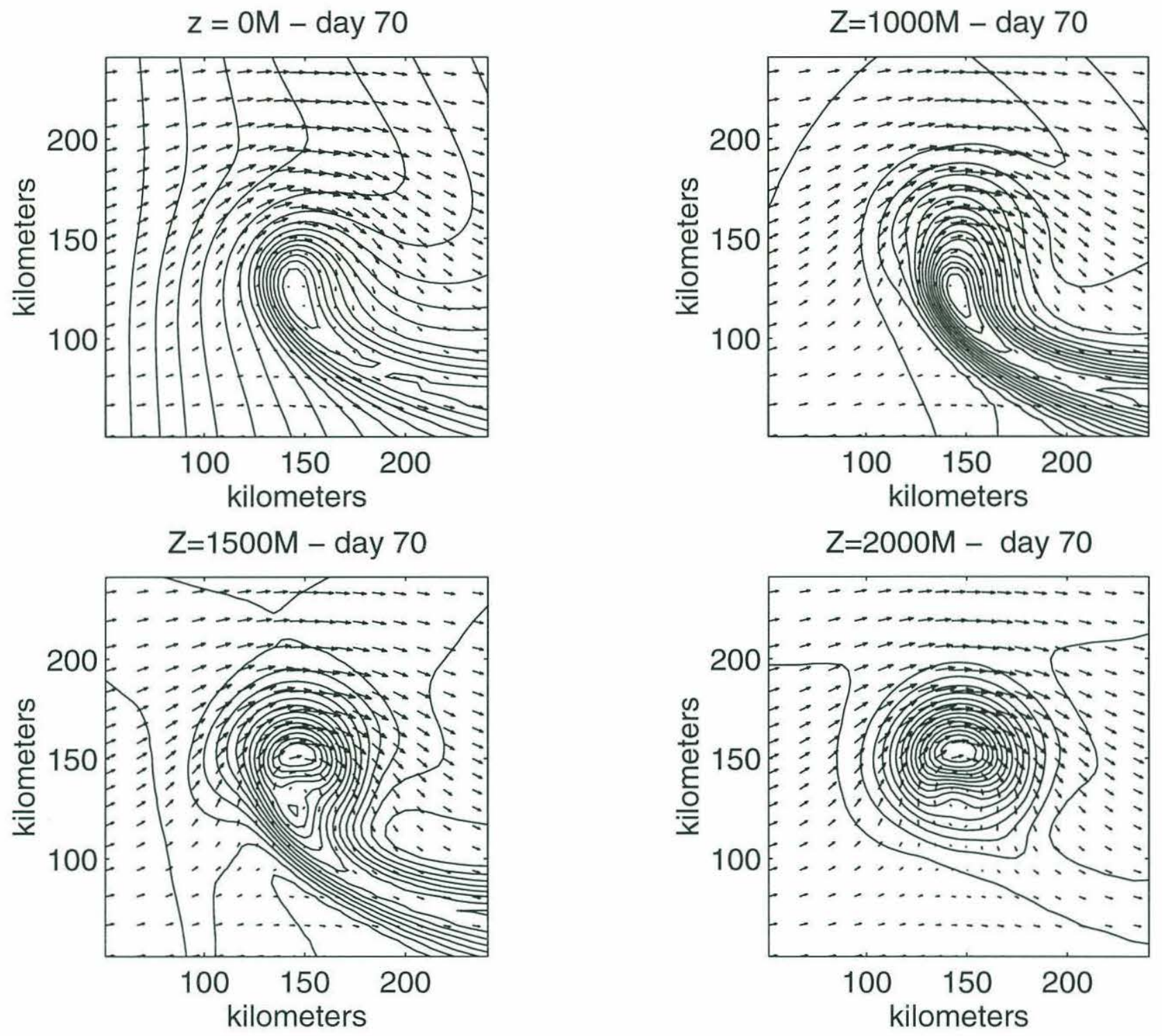

Figure 5.5: The steady-state horizontal velocity field at various depths in the topographic preconditioning run. Isopycnals are shown superimposed on the velocity vectors. In the upper water column, at the surface and at 500 meters depth, the mixed-layer deepening downtream from the inflow, the convective chimney over the seamount, and the dense wake are all visable. Deeper down, at 2000 meters depth, only the doming of isopycnals associated with the Taylor cap itself is visable. At 1500 meters depth, near the base of the chimney, both the chimney and the taylor cap are seen in the density field. 


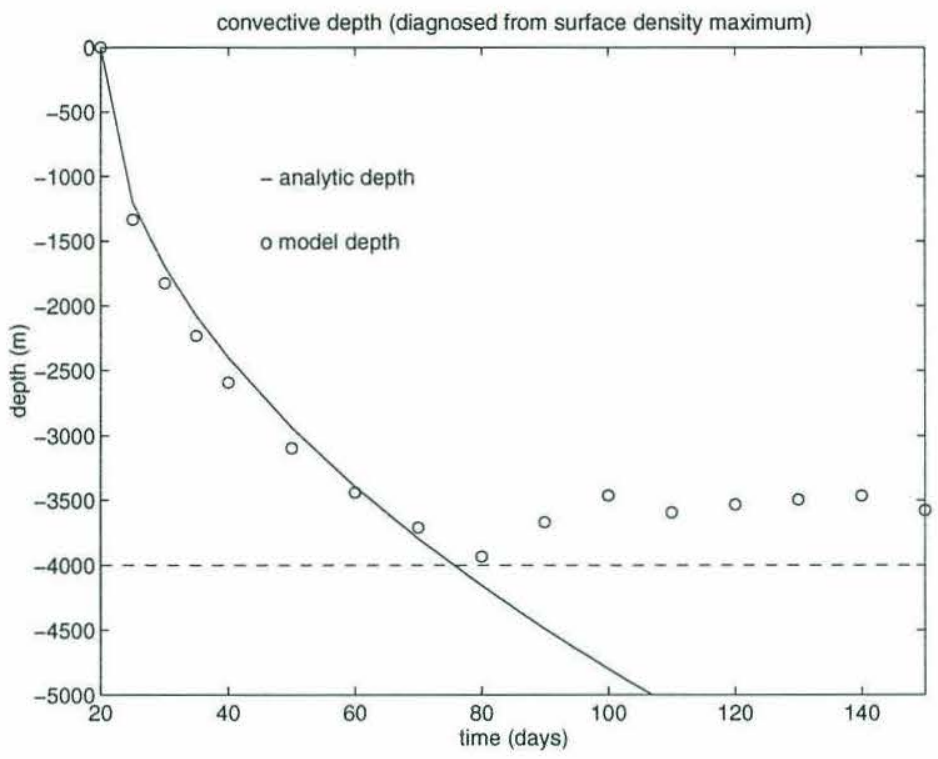

Figure 5.6: The time history of convective deepening of the topographically preconditionied chimney in run $\mathrm{C} 6$ is plotted with circles. The solid line shows the one-dimensional, nonpenetrative convection limit for comparison. After roughly 50 days of cooling, after the chimney has reached the bottom of the domain, a baroclinic eddy is shed and drifts downstream along the dense wake behind the chimney.

complete parameter dependency study is beyond the scope of this thesis, possible reasons for the remarkable stability of some such chimneys is investigated in section 5.3.

\subsection{Baroclinic Instability of Convective Chimneys}

The convective chimneys in runs D1 and $\mathrm{C} 4$ are of roughly the same radius and reach to similar depths and yet only the disk-cooling experiment is prone to baroclinic instability. The schematic in figure 5.8 demonstrates the essential cause of the unstable nature of the disk-cooled chimney. At depth, the low potential vorticity interior of the chimney compared to the ambient stratification produces a potential vorticity gradient oriented radially outward. At the surface, however, the positive density anomaly can be represented as a positive vorticity anomaly, giving a potential vorticity gradient oriented radially inward. The thin sheet of potential vorticity which replaces the surface density anomaly in this argument is generally refered to as a "Bretherton sheet" after Bretherton 

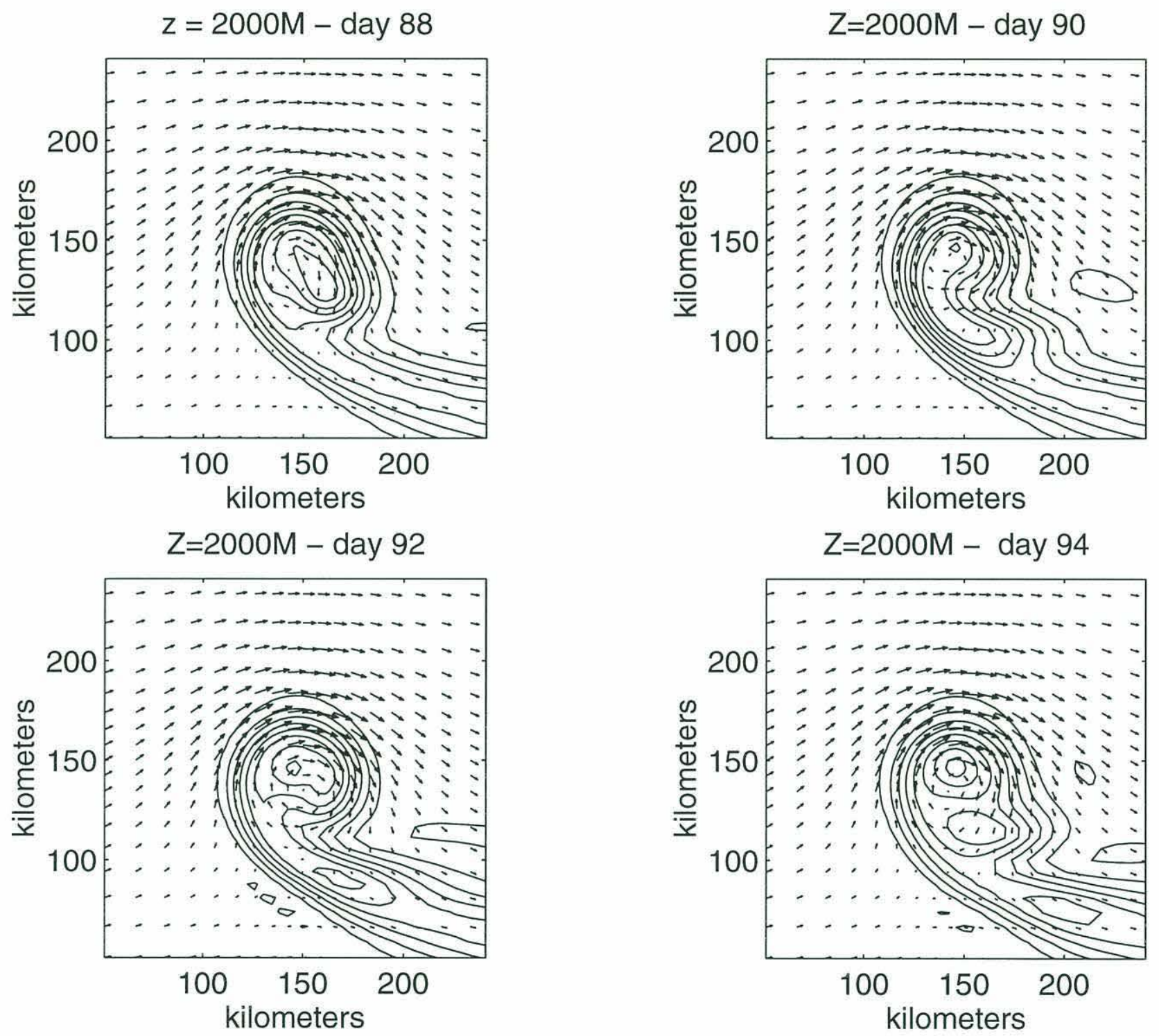

Figure 5.7: A time series showing the evolution of the horizontal velocity field at 2000 meters depth in run $\mathrm{C} 6$ with density contours superimposed showing the pinching off of a baroclinic eddy. 


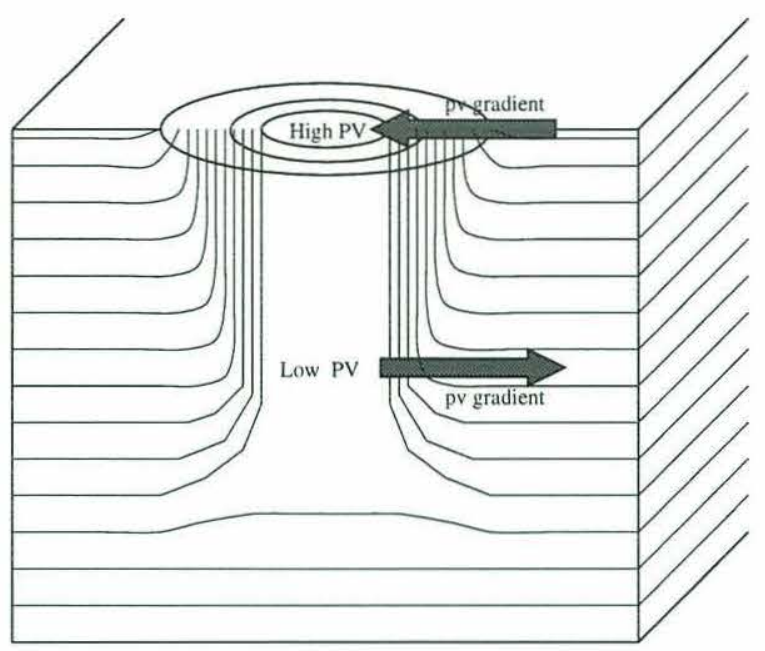

Figure 5.8: A schematic showing a cross section through a convective chimney. The change in potential vorticity gradient with height is a neccessary condition for baroclinic instability.

1966]. The change in sign of the potential vorticity gradient between the deep field and the surface Bretherton sheet is a necessary condition for baroclinic instability.

For comparison with this schematic representation, the potential vorticity can be diagnosed from model runs. I have calculated the potential vorticity on surfaces of constant depth rather than isopycnals, another source of some error. However, tests calculating potential vorticity on isopycnal surfaces show no qualitative differences in the resultant fields. The potential vorticity, as I have calculated it from the model fields, is defined as:

$$
p v=(\xi+f) \frac{\partial \rho}{\partial z}
$$

where the relative vorticity is given by:

$$
\xi=\frac{\partial v}{\partial x}-\frac{\partial u}{\partial y}
$$

This form of potential vorticity, which ignores the contribution from vertical shear terms, is a simplification of the full potential vorticity, $(f+\vec{\omega}) \cdot \nabla \rho$. Tests comparing the full potential vorticity with the simplified form in equation 5.3 show the neglected terms to be small in this case, justifying use of the simplified form. 
The potential vorticity field after 20 days of cooling at various depths in run D1 is shown in figure 5.9. Here the potential vorticity has been normalized by $f \frac{\partial \rho}{\partial z}$ of the initial stratification. Zero values inside the chimney are due to the complete homogenization of the water column by the convective adjustment scheme. The exterior values, far from the chimney in the horizontal as well as below the chimney, are exactly one since the stratification there has not been altered and the relative vorticity effects are confined to the rim current associated with the chimney walls. At 1000 meters depth, near the base of the chimney, the potential vorticity gradient is clearly radially outward. As expected, this chimney does break up due to baroclinic instability.

There are several key differences between the disk-cooled and topographically preconditioned chimneys. Some of these differences are examined in turn as possibly accounting for the difference in the instability properties for the two different chimneys. The upper two panels in figure 5.10 compare X-Z density slices for the disk-cooled (run D1) and topographically preconditioned chimneys (run C4). The first obvious difference between these chimneys is that the surface density gradient corresponding to the edge of the convective topographically preconditioned chimney is less sharply defined than for the disk-cooled chimney. Because the chimney is being generated by the presence of a Taylor cap, in a region with much less sharply defined edges, the surface density gradients, and potential vorticity gradients, are reduced. In order to assess the possible importance of the sharp gradient imposed by the rather unrealistic tophat shape of the surface cooling disk, a disk-cooling experiment with a wide cosine shaped taper down of the surface forcing region was performed.

In this run the disk over which the full buoyancy forcing is applied is still given a radius of 20 kilometers, as in run D1. Rather than using a tophat, the edges of the disk are tapered down to zero forcing with a cosine shaped curve. The cosine function operates over a radial distance between the edge of the original disk, at 20 kilometers radial distance, and a maximum of 80 kilometers from the center of the disk. An X-Z slice through the chimney formed below this cooling distribution is shown in the lower 

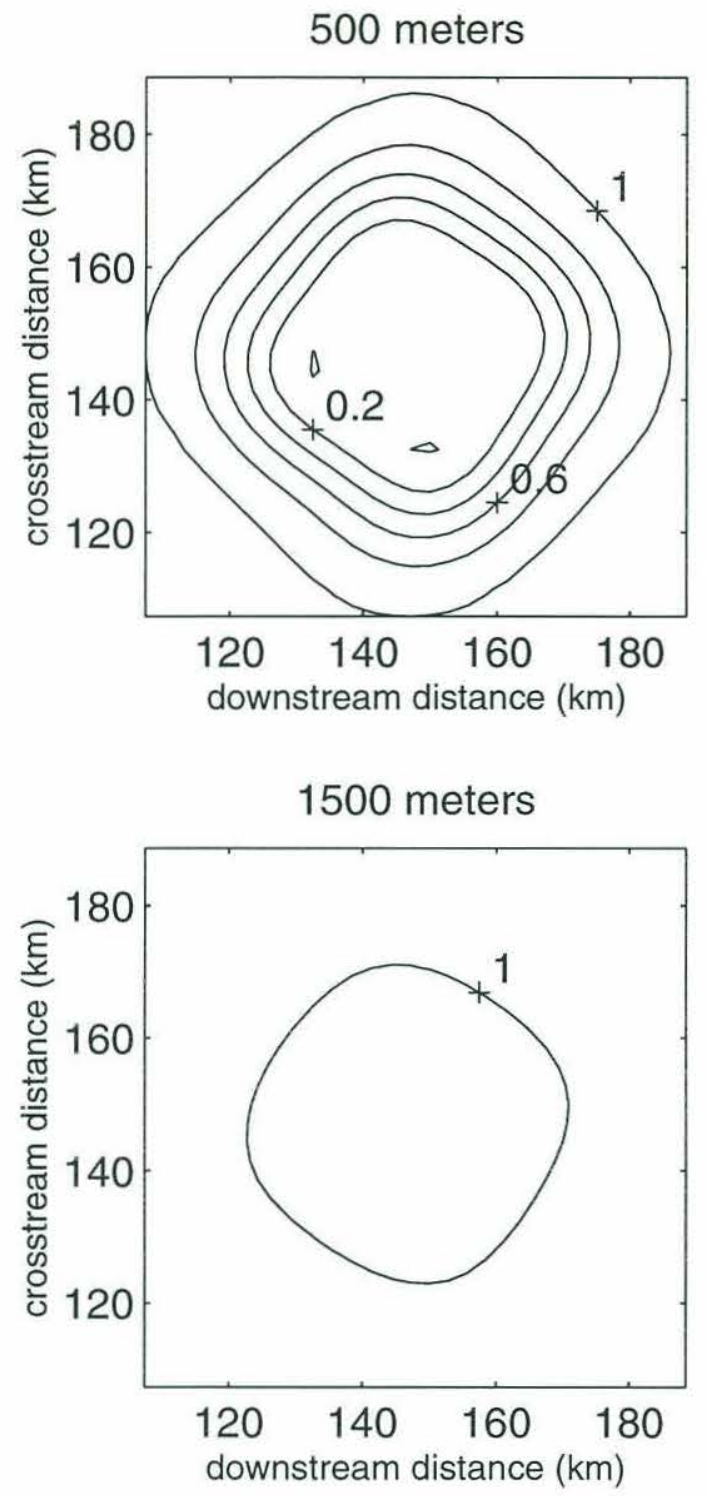
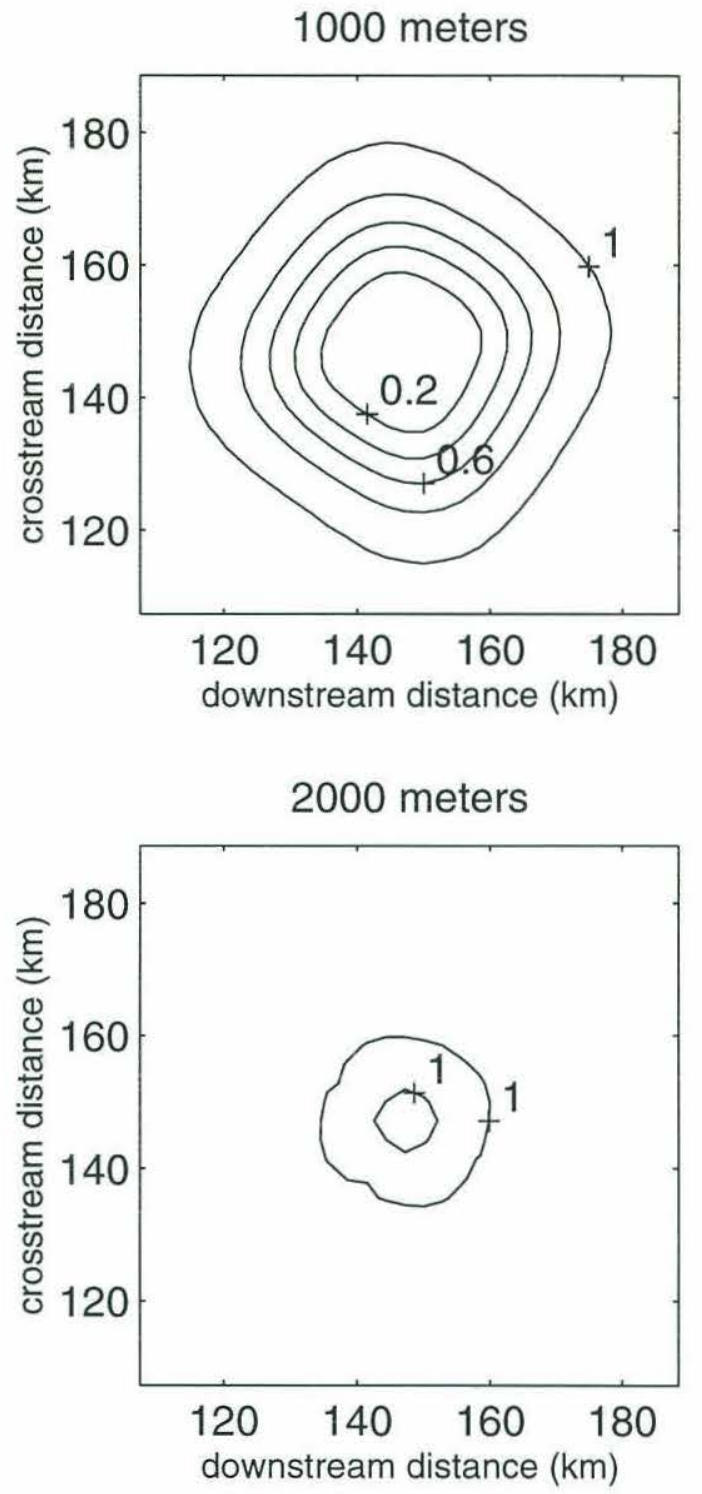

Figure 5.9: The potential vorticity field associated with the disk-cooled chiminey in run D1 at four different depths. Zero values in the center of the chimney indicate a complete vertical homogenization by the convective adjustment scheme. Outside the influence of the disk-cooling the initial values of one are unperturbed. Thus, the lack of contours in the lower two panels, below the base of the chimney, is simply a reflection of the homogeneity of the undisturbed initial field. In the upper two panels, the potential vorticity gradient in the chimney is radially outward as expected. 

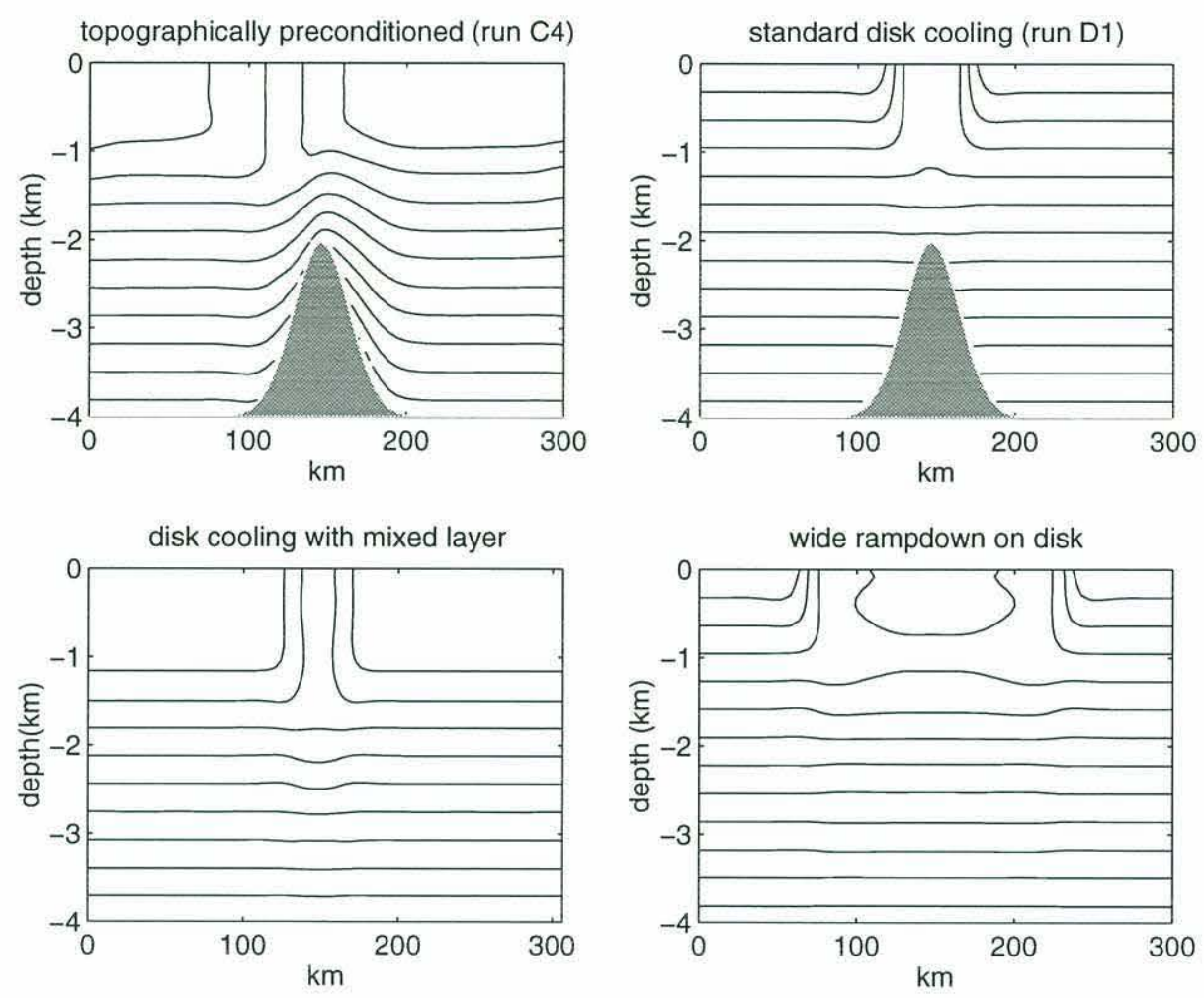

Figure 5.10: The upper two X-Z density slices compare the disk-cooled chimney (run D1) with the topographically preconditioned chimney (run C4). Two slightly modified disk-cooling runs, one with a wide taper down of the surface cooling around the edge of the disk and a second with an ambient mixed-layer depth outside the chimney itself, are also shown. 
right panel of figure 5.10 for comparison with runs D1 and C4.

The primary result from this run is that the chimney forced by a surface cooling distribution, which is tapered down over a wide zone around its edges, is still strongly baroclinically unstable. Due to the larger diameter of the chimney, a higher mode of instablity is evident as compared to the standard disk-cooling run, but the essential physics of baroclinic instablity shutting down the convective deepening remains. Thus, the artificial sharp edge to the cooling disk in this run and in run D1, as compared to run $\mathrm{C} 4$, does not appear to be the crucial condition for baroclinic instablility to occur.

A second characteristic of run $\mathrm{C} 4$ not found in D1, is the presence of a deep mixedlayer of low potential vorticity water outside the chimney itself. At 500 meters depth, for example, there is a radially outward gradient to the potential vorticity field in run D1, as seen in figure 5.9. Figure 5.11, by comparison, shows that the deep mixed-layer in run $\mathrm{C} 4$ causes the potential vorticity field at 500 meters depth to be nearly uniformly zero everywhere in the domain. The base of the chimney, however, is well below the ambient mixed-layer depth for the most part. Thus, there are radially outward potential vorticity gradients at 1000 meters depth. The high potential vorticity signal at 2000 meters depth, is due to lateral diffusion, which acts on sigma surfaces rather than isopycnals, leading to some crossisopycnal mixing and, consequently, a spurious pv source. Fortuitously this error is restricted to the deep Taylor cap region of very slow flow and domed isopycnals, and does not affect the use of pv as a diagnostic in the convective chimney.

In order to check if the presence of this deep mixed-layer outside of the chimney could inhibit the baroclinic instability, a disk-cooling run with an initial stratification including a 1000 meter deep ambient mixed-layer and otherwise identical to run D1 was performed. An X-Z density slice for the resulting chimney is shown in the lower left panel of figure 5.10. The instability of this chimney is qualitatively indistinguishable from the mode four growth of the standard disk-cooling run (D1), indicating that the presence of the low potential vorticity mixed-layer is not inhibiting the chimney instability process.

Finally, a crucial difference between the disk-cooling chimney and the topographically 
500 meters

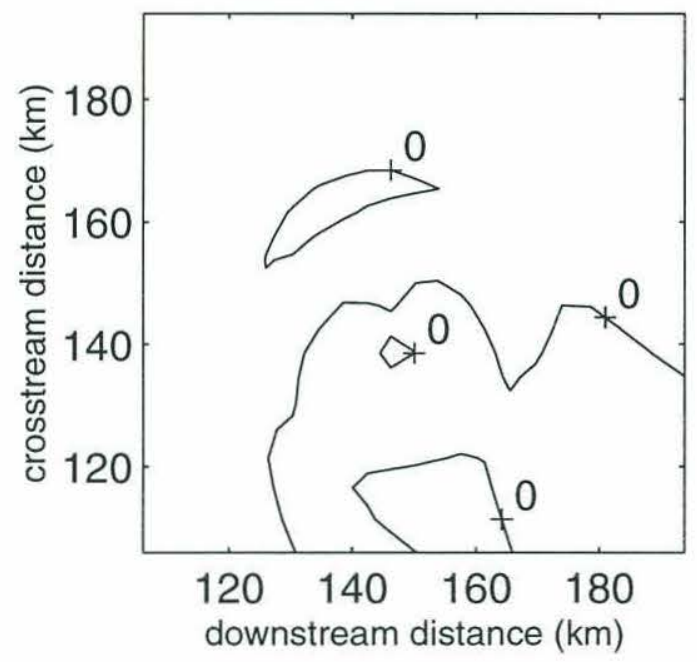

1500 meters

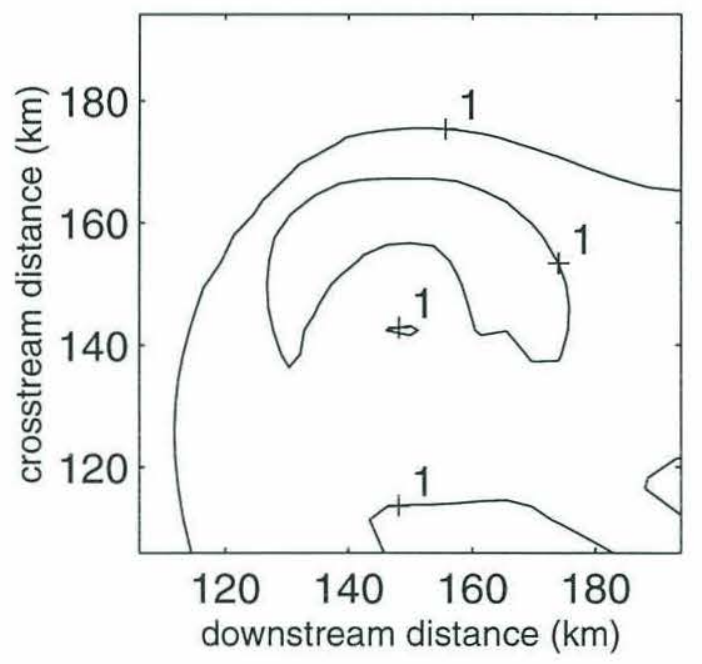

1000 meters
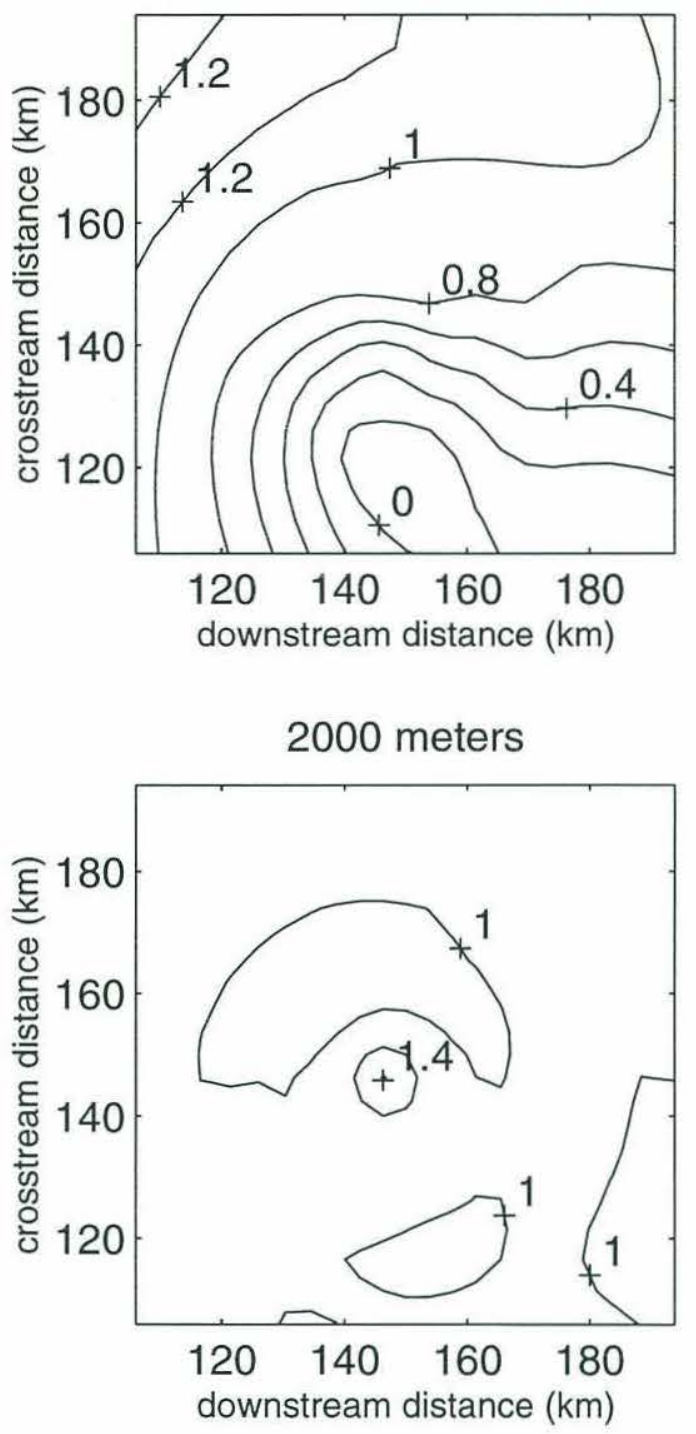

Figure 5.11: The potential vorticity field associated with the topographically preconditioned chiminey in run $\mathrm{C} 4$ at four different depths. Potential vorticity has been scaled by $f \frac{\partial \rho}{\partial z}$ of the background stratification. Zero values in the surface mixed-layer and the center of the chimney indicate a complete vertical homogenization by the convective adjustment scheme. Outside and below the influence of the disk-cooling the initial values of one are unperturbed. The potential vorticity gradient in the chimney at 1000 meters depth is radially outward in part. Although not as simple as the corresponding picture for the disk-cooled chimney, the neccessary condition for instability does exist. 
preconditioned one is the presence of the barotropic mean flow in the latter case. As evident in figure 5.5, there is a strong zonal flow in the region of the horizontal density gradients associated with the chimney walls. One possible explanation for the lack of growing instabilities on this horizontal density gradient is that, although the conditions for unstable growth do exist, the mean flow is so strong that any growing disturbances are advected downstream faster than they can grow locally. For comparison, in the diskcooling runs the velocity field, shown in figure 5.2 , is entirely recirculating around the chimney.

In order to test whether this advective effect is indeed responsible for the inhibition of instability one can compare estimates of the timescale for instability growth with the advective timescale given by the magnitude of the mean flow and the horizontal scale of the chimney. One rough estimate for the growth rate of the most unstable wave comes from the original estimate by Eady [1949] for parallel flow with uniform stratification:

$$
k c_{i} \propto \frac{0.3 f}{\sqrt{R}_{i}} \propto \frac{0.3 f \frac{\partial u}{\partial z}}{N} .
$$

With an associated timescale:

$$
T_{\text {eady }}=\frac{1}{k c_{i}}
$$

Taking the approximate shear from the Taylor cap flow from run $\mathrm{S} 2$ to be $5 \mathrm{~cm} / \mathrm{sec}$ over 2000 meters, as seen for example in figure 3.6, and using the initial background value of $5.556 \times 10^{-4}$ for $N$, gives a Richardson number of approximately 500 . The corresponding growth rate of the fastest growing unstable mode according to equation 5.6 is slightly over 8 days.

Another estimate of the growth rate of instabilities can be obtained by examining the numerical growth rate of the mode four instability on the disk-cooling run, which has basic chimney characteristics very similar to this topographic chimney. Figure 5.2 shows the development of this instability with time. The growth rate for this instability appears to be on the order of 10 days.

The advective timescale for the mean flow to advect disturbances beyond the chimney 
region is simply a function of the chimney radius and magnitude of the mean flow.

$$
T_{\text {advective }}=\frac{L}{U}
$$

Substitution of a background flow speed of $0.2 \mathrm{~m} / \mathrm{sec}$ and the approximate radius of the chimney, $20 \mathrm{~km}$, into equation 5.7 leads to an advective timescale of approximately 1 day. Note that, if anything, this is an overestimate of the advective timescale since the flow is actually significantly accelerated above the background levels in the vicinity of the northern edge of the chimney as shown in figure 5.5.

Both estimates of the timescale for the growth of baroclinic instabilities are an order of magnitude greater than the advective timescale. This indicates that the likely cause for the apparent stability of the topographic chimney is not a lack of conditions for unstable growth. Rather, the growing instabilities are simply being advected downstream, out of the region where the conditions allow for growth, faster than they can grow locally within that region.

Given the complicated structure of the convective chimney superimposed on Taylor cap flow it is difficult to assess the instability growth rate with more accuracy than these simple scaling arguments supply. One possibility, however, is to look at the semicircle theorem for parallel quasigeostrophic flow, which supplies bounds on the growth rate of the fastest growing baroclinic mode. Given a linear, quasigeostrophic, parallel flow over a flat bottom and assuming a standard waveform disturbance of the form,

$$
\phi=\Re \operatorname{eal}\left(\Phi(y, z) e^{i k\left(x-c_{r} t\right)} e^{k c_{i} t}\right),
$$

the growth rate of any given mode is just $k c_{i}$. The semicircle theorem [e.g. Pedlosky 1987], which gives bounds on the possible values of $c_{i}$, is:

$$
c_{i}^{2} \leq\left(\frac{U_{\max }-U_{\min }}{2}\right)^{2}+\frac{\beta}{k^{2}+\pi^{2} / 4}\left(\frac{U_{\max }-U_{\min }}{2}\right)
$$

For the case of an f-plane this reduces to:

$$
c_{i}^{2} \leq\left(\frac{U_{\max }-U_{\min }}{2}\right)^{2},
$$


which can be calculated as a function of downstream distance using the steady-state velocity from the $\mathrm{C} 4$ model run. A time scale for the growth rate can now be calculated by assuming that the fastest growing mode is on the order of four times the Rossby radius (after Pedlosky [1987]):

The wavenumber, $\mathrm{k}$, is then simply

$$
k=\frac{2 \pi f}{4 N H} .
$$

These formulae for $k$ and $c_{i}$ give another estimate of the bound on local growth rate, as a function of downstream distance. This bound is plotted in figure 5.12 along with the local advective timescale. Again, even in the region of maximum growth rate, the advective timescale is significantly faster than that for instability growth. Obviously, this theory is not strictly applicable to the steady-state flow field of run $\mathrm{C} 4$, since the flow is not parallel, is nonlinear and is not quasigeostrophic. However, in addition to the preceding scaling arguments, it provides an indication that the instability growth rates are likely to be too small to lead to a breakup of the convective chimney.

\subsection{Conclusions}

The final depth to which convection penetrates is examined in this chapter. A run is made in which a chimney is generated without topographic preconditioning through the application of a disk-shaped cooling function at the surface. This disk-cooling run reproduces the results of Visbeck et. al. in which horizontal fluxes of heat associated with baroclinic instability of the rim current shut down the deepening of the convective chimney. This hydrostatic model and simple convective adjustment scheme agree with the scalings Visbeck et. al. have derived using a much higher resolution nonhydrostatic model and laboratory experiments.

In contrast to the disk-cooled chimney, the topographically preconditioned chimneys catalogued in Chapter 3 are remarkably stable. Scaling arguments show that the expected 


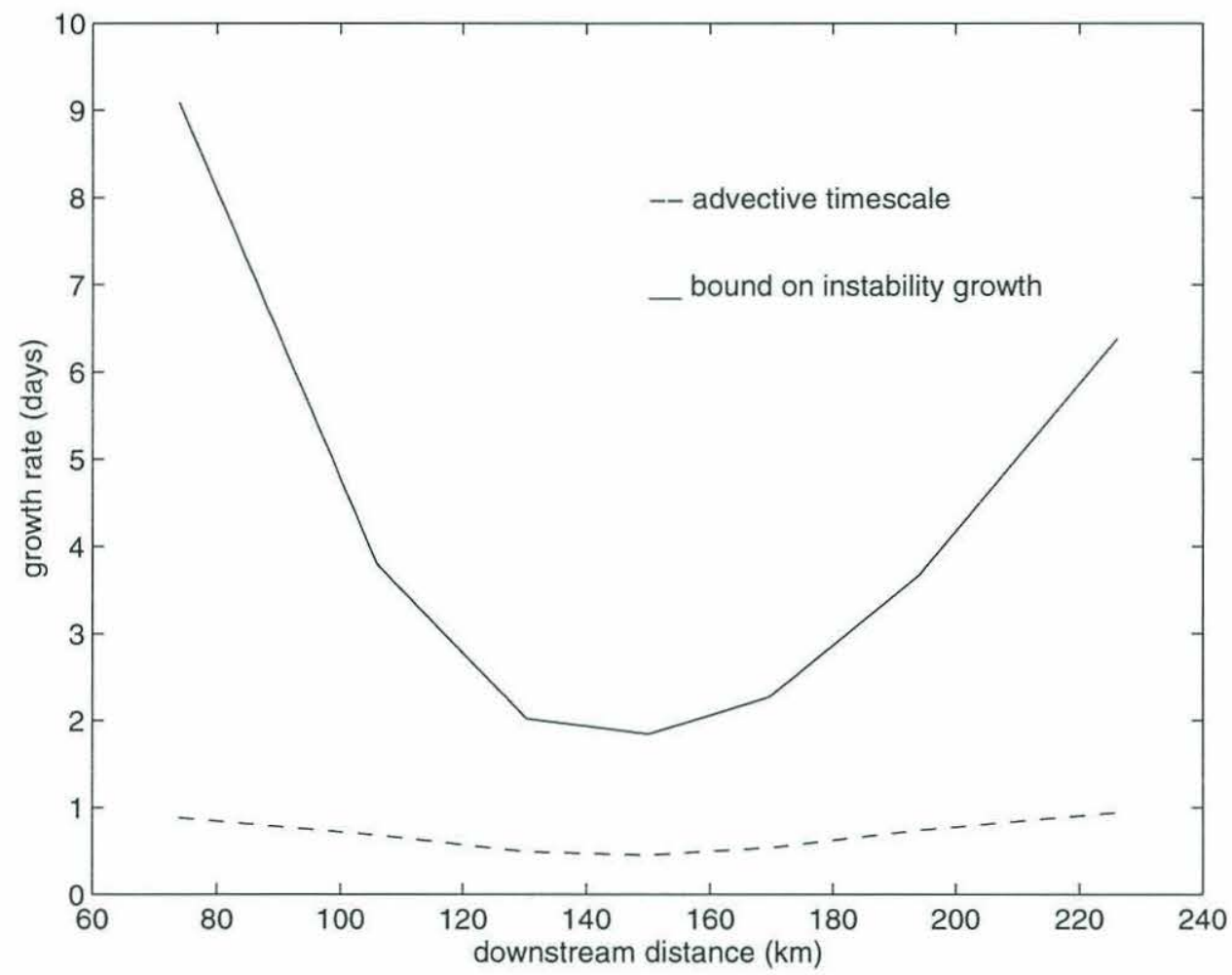

Figure 5.12: A comparison of the advective time required for the mean flow to flush through the region of the chimney walls and the quasigeostrophic bound on the time scale of instability growth derived from the steady-state velocity field in run $\mathrm{C} 4$ as a function of distance from the inflow. 
time scale for the growth of baroclinic instabilities is about an order of magnitude larger than the time for the mean flow to advect particles past the chimney feature. Thus, in the context of a topographically trapped chimney, any initial perturbation to the chimney is advected downstream beyond the region where conditions for growth exist much faster than it can grow locally. In addition to the scaling arguments, quasigeostrophic theory also suggests that the growth rate, relative to the advective timescale, is too slow for instabilities to develop.

The inhibition of instability growth by mean flow advection occurs in most of the runs which were run out to steady-state. In only one run, with a low enough background stratification for the convection to reach the bottom of the domain, were baroclinic eddies generated. These eddies were pinched off in the region of the dense wake downstream of the Taylor cap, rather than the circularly symmetric modal pattern seen in the diskcooled run.

One might expect weaker mean flows to allow baroclinic instability to develop. However, if there is no mean flow, there is no Taylor cap. In other words, in the limit of weak mean flows, there is no longer topographic preconditioning, and no chimney will form. As the mean flow is increased enough to allow a Taylor cap and convective chimney to form, it becomes too strong to allow baroclinic instabilities to grow locally. Thus the inhibition of baroclinic instability is likely to be robust within the range of mean flow speeds wherein the topographic preconditioning mechanism is applicable. Consequently, inferences about the role of eddy fluxes in oceanic convection based on the more common disk-cooling type of experiment, which parameterizes the preconditioning process by setting the chimney scale equal to the surface forcing scale, should be treated with care. If the preconditiong process being parameterized in such disk-cooling experiments is a topographic mechanism such as that modeled in this thesis, the role of baroclinic eddies is probably being overemphasized.

In the case of these stable topographically preconditioned chimneys it is the horizontal advection of heat across isopycnals in the upper water column which eventually balances 
the cooling over the surface of the chimney and shuts down the convective deepening. This steady-state depth can be somewhat deeper than the final depth of disk-cooled chimneys given the same rate of cooling and initial stratification. In addition, even after the chimney has stopped burrowing into deeper water, its stability allows for further ventilation of the greatest density class that it has reached. In addition to the initial preconditioning effect of the topography, inhibition of baroclinic instability may also allow enhanced ventilation of deep waters in regions of mean flow over isolated bottom topography. 


\section{Chapter 6}

\section{Conclusions}

\subsection{Summary of the thesis}

In this thesis I have investigated the role that isolated topography in the presence of a mean flow can have on convective processes forced at the surface of the ocean. It is shown that topography can serve to select a location where convection will preferentially occur. This preconditioning is primarily due to isolation of the fluid over the seamount from horizontal fluxes of heat by the mean flow. When surface intensification of the background stratification is included, doming of isopycnals associated with the anticyclonic Taylor cap circulation also plays a preconditioning role. In addition to selecting the location for chimney formation, the topography sets an oceanographic scale for the chimney, despite the generally much larger scale of atmospheric forcing. Finally, the presence of a mean flow past the topographically trapped chimney can inhibit the breakup of the chimney due to baroclinic instability.

Chapter 3 outlines a simple set of experiments where a Taylor cap is spun up over an isolated Gaussian seamount in a constant background stratification using a primitiveequation model. Uniform negative buoyancy forcing is applied over the surface of the steady-state Taylor cap. The surface mixed-layer away from the influence of topography deepens with a square root dependence on distance downstream from the inflow, as 
predicted by simple non-penetrative convection theory acting over a time equal to the advective time required for the inflow to reach that downstream location. Over the seamount, where the flow is trapped and is not being ventilated by the mean flow from upstream, the mixed-layer deepens significantly further than elsewhere in the domain. Within the topographically trapped chimney the deepening initially follows the onedimensional, analytic limit for the maximum convective deepening that could occur over the total period of time for which the cooling is applied. Downstream of the topography there is a dense wake of deep mixed-layer where particles are slowed in the region of the seamount, but not trapped, and experience the surface cooling for a longer period of time as a result.

The essential physics of this topographic preconditioning is the interaction of the surface mixed-layer with the region of topographically trapped fluid. This interaction is increased at low Rossby number since the slower flow speeds tend to increase mixed-layer depth and also increase the height to which the Taylor cap reaches. Similarly, lower stratification parameter, $N / f$, both increases mixed-layer penetration and Taylor cap height. Increasing the convective Rossby Number (non-dimensionalized surface forcing) increases the mixed-layer depth, thus increasing the preconditioning effect without directly effecting the Taylor cap itself. Seamount fractional height brings the region of trapped fluid higher, increasing the preconditioning effect, without directly affecting the mixed-layer depth. The dependence on seamount aspect ratio, the final non-dimensional parameter, was not investigated.

In chapter 4 the preconditioning effect of domed isopycnals is investigated. For uniform stratification, such as that used for the runs in Chapter 3, it is shown that domed isopycnals do not precondition for deeper convection. In fact, if anything the higher stratification required if the isopycnals dome up while holding surface temperature constant can actually decrease the depth of penetration. Allowing for a nonuniform, surface intensification in the stratification is the key to having domed isopycnals play a preconditioning role. An analytic formula for exponential stratification analogous to the well 
known one-dimensional, non-penetrative convection into uniform stratification is developed.

As predicted by this analytic formula, the region of isopycnal doming will be the first place where the pycnocline ventilates. Once the deep water has been ventilated, the convection rapidly penetrates into the deep region of weaker stratification. A steady-state Taylor cap is spun up with an exponential stratification identical to that used for the analytical calculation. This run confirms that a nonuniform stratification significantly enhances the preconditioning over the topography by allowing the domed isopycnals to play a role. The depth of convection in the numerically modeled chimney is in good agreement with the implicit analytical prediction.

The shutdown of the deepening of convective chimneys is investigated in Chapter 5. Previous modeling of convection using high resolution nonhydrostatic model have been forced by disk shaped surface cooling distributions, setting the scale of the oceanic response with the surface forcing. These chimneys deepen initially following the onedimensional, non-penetrative convection limit. However, they are prone to breakup due to baroclinic instability of the chimney. The horizontal fluxes of heat associated with the baroclinic instability are sufficient to shut down the convective deepening. One such disk cooled run is reproduced using the SPEM model, and compared with a topographically preconditioned chimney holding all other parameters constant.

One remarkable difference between the chimney generated by a disk of cooling at the surface and that in which the topography sets the oceanographic scale is a lack of instability in the topographically trapped chimney. The principal mechanism responsible for instability, a change in the sign of the potential vorticity gradient with height, is present in both chimneys. However, in the case of the topographically preconditioned chimney, the expected time scale for the growth of baroclinic eddies is an order of magnitude smaller than the advective timescale associated with mean flow past the chimney. Thus, any perturbation that begins to grow on the topographically preconditioned chimney is advected downstream much faster than it can grow locally. The shutdown of deepening, 
in the topographically preconditioned case, occurs as a result of fluxes of heat by the mean flow rather than baroclinic eddies.

Synthesizing the results of chapters 3,4 , and 5 it is clear that isolated topography can potentially play an important preconditioning role by setting the location and scale of oceanic convective chimneys. The basic preconditioning effect is an interaction between the surface mixed-layer and the trapped fluid in the Taylor cap. The preconditioning can be further enhanced by incorporating a surface intensification of the background stratification, which allows the pycnocline to be ventilated first in the region of domed isopycnals associated with the Taylor cap. Finally, the convection can penetrate deeper and ventilate for a longer period of time because of the stability of the chimney due to the presence of a mean flow.

Given these conclusions, it is worth recalling that the process oriented modeling experiments in this thesis were motivated by the presence of high levels of convection over Maud Rise in the Weddell Sea. The range of Rossby numbers, stratification parameters, and seamount fractional heights ysed are reasonable in the context of flow over Maud Rise. Similarly, the range of buoyancy fluxes applied is consistent with the mean level of wintertime cooling in the Weddell sea. Much stronger buoyancy fluxes, perhaps as high as $1000 \mathrm{~W} / \mathrm{m}^{2}$, are of course likely to occur at times, especially when strong low pressure systems force divergent ice motion and open leads in the sea ice. These short term bursts of cooling, operating on time scales of a few days, are probably important in selecting the exact location and time of initiation of convective events over Maud Rise.

In order to resolve the Rossby radius of deformation, while maintaining the ability to run the model many times through a wide range of parameter space, it was necessary to use a seamount aspect ratio which is not appropriate for Maud Rise. The model seamounts have a horizontal length scale of only 25 kilometers, whereas Maud Rise has a horizontal scale of more like 100 kilometers. It is assumed that the use of a wider seamount would lead to the same basic processes as the modeled, more narrow, seamounts in this study. 
In addition, this study neglects salinity as a dynamic variable as well as the role of sea ice, both of which certainly play important roles in convection occurring over Maud Rise. Another criticism of the dynamical picture of topographic preconditioning presented in this thesis is the use of a purely barotropic mean flow. The volume flux through the model domain, a uniform $0.2 \mathrm{~m} / \mathrm{sec}$ over 300 kilometers width and 4 kilometers depth, amounts to an enormous and unrealistic 240 Sverdrups. In addition, in the real ocean one does not find flow completely devoid of shear in the vertical (or horizontal). In response to this potential drawback, it should be noted that the volume flux, although admittedly very large, includes a great deal of flow which travels through the domain well beyond the region affected by the presence of the seamount. The volume flux past a region of 40 kilometers width, around the seamount, is a somewhat more palatable 32 Sverdrups.

The issue of how inclusion of vertical shear will affect the topographic preconditioning problem is an interesting one. To first order, the effect of vertical shear will be to decouple the surface mixed-layer from the bottom trapped Taylor cap, thus reducing the effectiveness of the topographic preconditioning mechanism. However, weaker flows at depth, as are found in the Weddell Gyre for example, would allow the Taylor cap to penetrate higher in the water column, thereby increasing the likelihood of interaction with the surface mixed layer. Another interesting possibility to consider is the case in which mean flow at depth is strong enough to spin up a steady-state Taylor cap, thus enabling a chimney to form, but too weak to inhibit baroclinic instability of the chimney. This would perhaps lead to an interesting depth dependent structure as baroclinic instability leads to breakup of the base of the chimney.

Despite the numerous potentially important processes not captured by this model, the first order picture of topographic preconditioning which is presented is probably robust. A more complicated scenario, including salinity, vertical shear in the inflow, sea ice, and more realistic topography would certainly lead to quantitatively different levels of convection. However, the qualitative picture of Taylor cap interaction with the surface mixed layer as a preconditioning mechanism, as well as the first order parameter 
dependencies for this process, are likely to remain valid. Thus, while it does not provide a quantitative, or prognostic, measure of convection in the Weddell sea, this thesis does outline the likely basic physical mechanism involved as well as how the process depends on some of the more basic oceanographic parameters.

\subsection{Future Work}

There are several possible extensions to this work. Continuing the parameter study of chapter 3, the dependence on seamount aspect ratio could be investigated. Although very wide seamounts would be expensive to run while still resolving the internal Rossby radius, the seamount width is likely to be an important parameter since the height of topographic influence, $N / f L$, is dependent on the horizontal scale of the topography.

The inhibition of baroclinic instability by the mean flow is also an area where further work could be concentrated. At very low Rossby number it is not possible to generate a steady-state Taylor cap. Instead, the initial spinup leads to topographically trapped anticyclonic and cyclonic vortices which co-rotate around the seamount. Thus, it is not easy to investigate the regime where mean flow is weak enough to allow baroclinic instabilities to develop in the context of topographically preconditioned chimneys. However, the problem can be examined quite readily in the context of simpler disk cooling experiments.

For example, consider cooling applied over a disk shaped region with uniform background stratification and a barotropic mean flow. The limit of zero mean flow is simply the disk cooled chimney from chapter 5 , which deepens below the disk until convection is shut down by lateral fluxes of heat associated with baroclinic instability of the chimney rim current (recall run D1, shown in figure 5.2). Given weak enough mean flows one would expect little deviation from this dynamical regime.

Indeed, a test disk cooling identical to run D1 except with a $1 \mathrm{~cm} / \mathrm{sec}$ mean flow applied is shown in figure 6.1. Although a slight downstream elongation of the rim current is apparent, the baroclinic instability is clearly in evidence. For comparison, a second run 

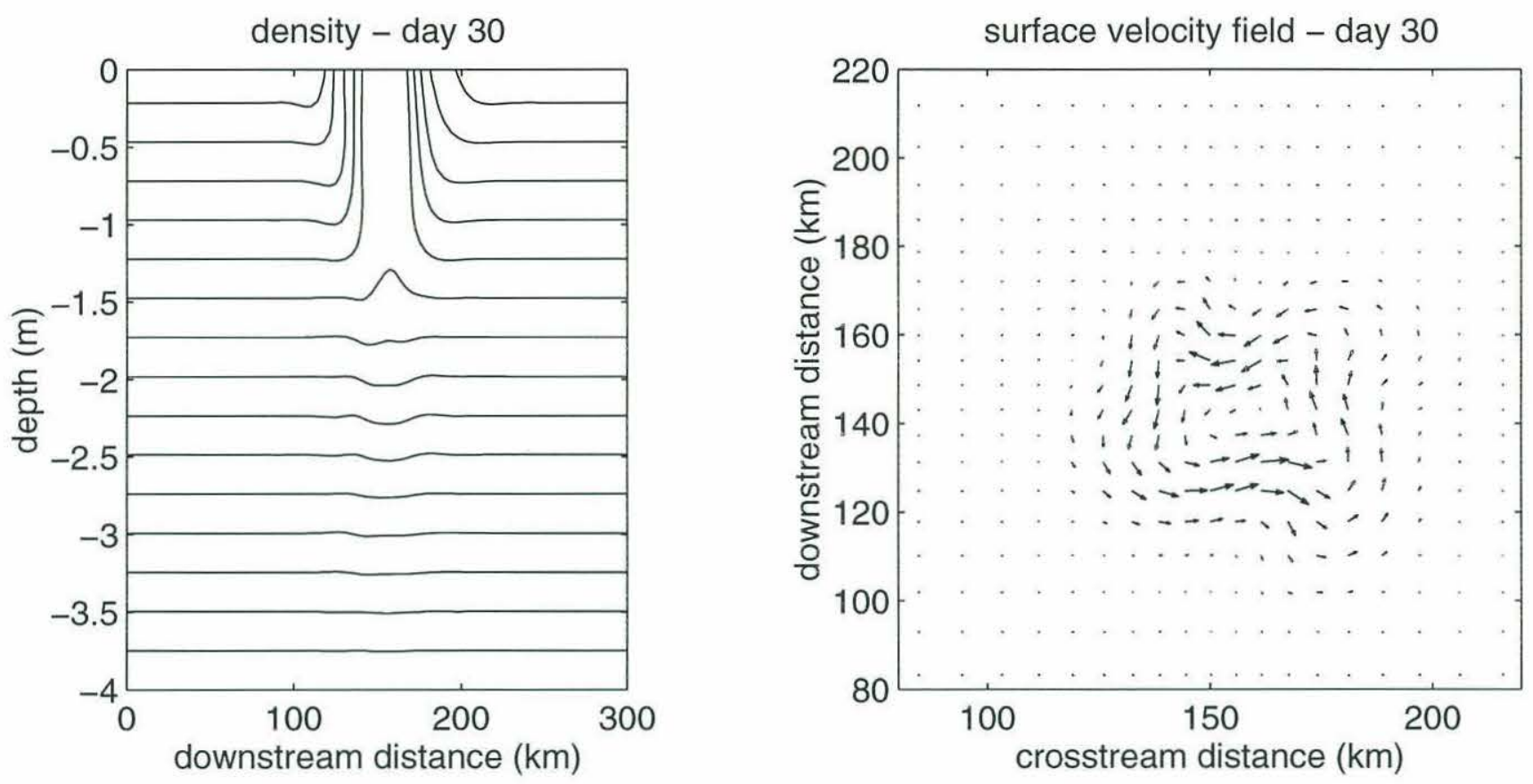

Figure 6.1: A Y-Z density slice and the surface velocity field for a disk cooling experiment with a mean flow of $1 \mathrm{~cm} / \mathrm{sec}$

with a $10 \mathrm{~cm} / \mathrm{sec}$ mean flow is shown in figure 6.2 . In this case the mixed-layer deepens with downstream distance below the cooling and extends at constant depth from the downstream edge of the cooling disk to the domain exit. This mixed-layer deepening is certainly not chimney shaped, and, in addition, show no signs of baroclinic instability. These two runs are presented here simply as one possible avenue of research into how to understand the inhibition of baroclinic instability of convective chimneys by the mean flow. Perhaps, in the regime where the rim current associated with the convective deepening is stronger than the background flow, perturbations are not advected downstream and local unstable growth becomes possible. A study of the dependence of such a critical mean flow speed on the other parameters in the problem, such as stratification and buoyancy forcing, would be a straightforward and elucidating extension to the thesis.

Finally, another interesting extension of this process oriented study would be to try and model the Maud Rise scenario more realistically in order to get bounds on the rate of formation of deep water there and perhaps outline the tendency of the system to flip 

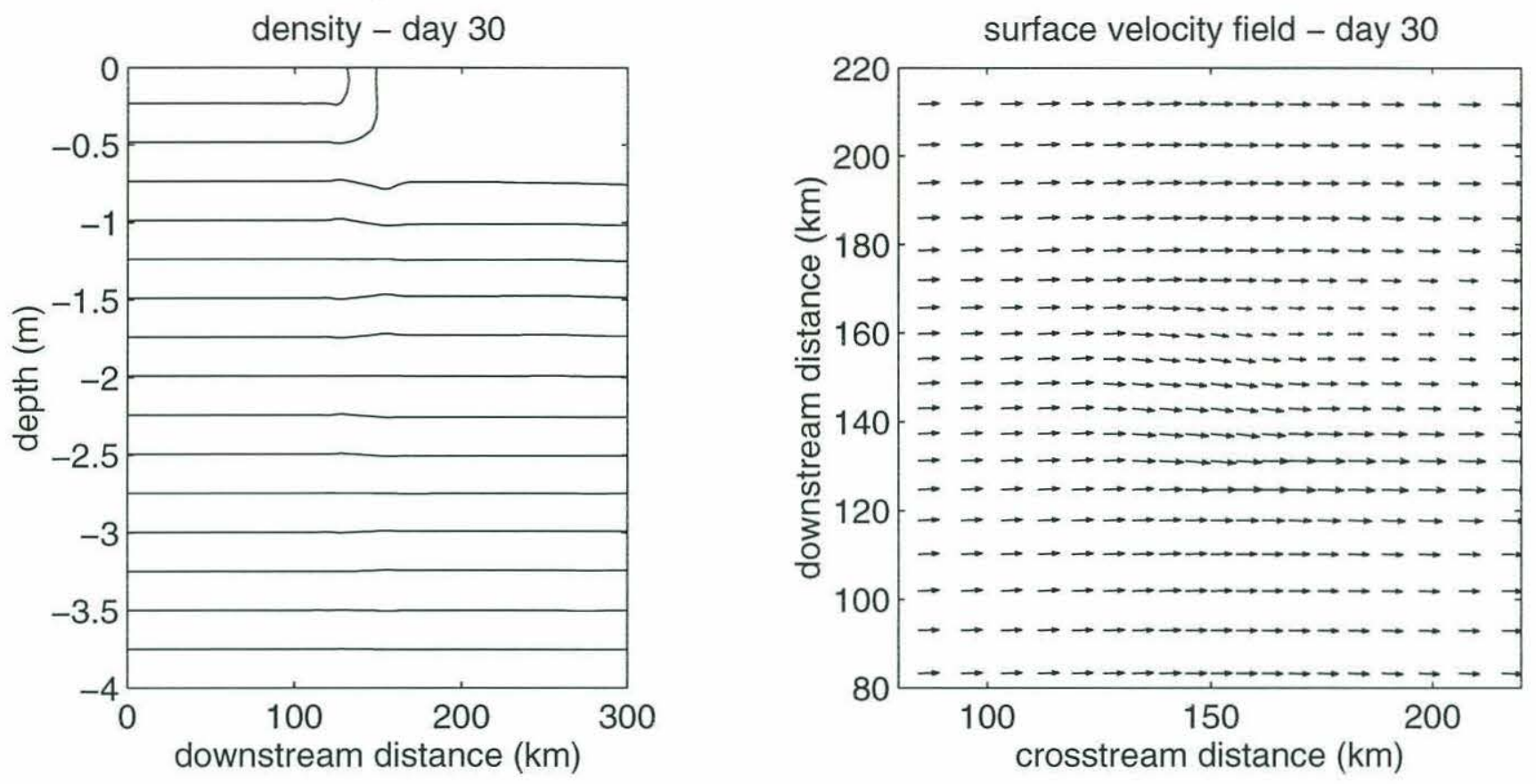

Figure 6.2: A Y-Z density slice and the surface velocity field for a disk cooling experiment with a mean flow of $10 \mathrm{~cm} / \mathrm{sec}$

from polynya to non-polynya modes. Moving towards such a realistic study would first entail the inclusion of salinity in the equation of state, vertical and horizontal shear in the incoming mean flow, and a sea ice model. 


\section{REFERENCES}

Bennet, A. (1975) Open Boundary Conditions for Dispersive Waves, Journal of the Atmospheric Sciences, 33, 176-182.

Bersch, M. (1988) On deep convection in the Weddell Gyre, Deep Sea Research, 35, 1269- 1296.

Bretherton, F. (1966) Critical layer instability in baroclinic flows, Quarterly Journal of the Royal Meteorological Society, 92, 325-334.

Brugge, R., H.L. Jones, and J.C. Marshall (1991) Non-hydrostatic ocean modelling for studies of open-ocean deep convection, Deep Convection and Deep Water Formation in the Oceans, Chu and Gascard (Editors), 325-340.

Buckingham, E. (1914) On physiclly similar systems; illustrations of the use of dynamical equations, Phys. Rev. IV, 4, 345.

Chapman, D. (1985) Numerical Treatment of Cross-Shelf Open Boundaries in a Barotropic Coastal Ocean Model, Journal of Physical Oceanography, 15, 1060-10756.

Chapman, D., and D. Haidvogel (1992) Formation of Taylor caps over a tall isolated seamount in a stratified ocean, Geophysical and Astrophysical Fluid Dyanamics, 64, 31-65.

Eady, E. (1949) Long waves and cyclone waves, Tellus, 1, 33-52.

Foldvik, A., T. Gammelsrod, and T. Torresen, (1985) Hydrographic observations from the Weddell Sea during the Norwegian Antarctic Research Expedition 1976/77, Polar Research, 3, 177-193.

Foldvik, A., T. Gammelsrod, and T. Torresen, (1985b) Physical oceanography studies in the Weddell Sea during the Norwegian Antarctic Research Expedition 1978/79, Polar Research, 3, 195-207.

Gloerson, P., W. Campbell, D. Cavalieri, J. Comiso, C. Parkinson, and H. Zwally (1992) Arctic and Antarctic Sea Ice, 1978-1987: Satellite Passive-Microwave Observations and Analysis, NASA SP-511, 290 pgs.

Gordon, A.L. (1978) Deep Antarctic Convection West of Maud Rise, Journal of Physical Oceanography, 8, 600-612.

Gordon, A.L. (1982) Weddell Deep Water Variability, Deep Sea Research, 40,supplement, 199-217. 
Gordon, A., and B. Huber (1990) Southern Ocean Winter Mixed Layer, Journal of Geophysical Research, 95, C7, 11655-11672.

Green, J. (1970) Transfer properties of the larege-scale eddies and the general circulation of the atmosphere, Quarterly Journal of the Royal Meteorological Society, 96, 157185 .

Haidvogel, D., J. Wilkin, and R. Young (1991) A Semi-spectral Primitive Equation Ocean Circulation Model Using Vertical Sigma and Orthogonal Curvilinear Horizontal Coordinates, Journal of Computational Physics, 94, 151-185.

Hakkinen, S. (1988) A Note on Chimney Formation in Ice Edge Regions, Journal of Geophysical Research, 93, 8279-8282.

Hermann, A.J. and W.B. Owens (1993) Energetics of Gravitational Adjustment for Mesoscale Chimneys, Journal of Physical Oceanography, 23, 346-371.

Hide, R., and A. Ibbetson (1966) An experimental study of Taylor columns. Icarus, 5, 279- 290.

Hide, R., A. Ibbetson, and M.J. Lighthill (1968) On the slow transverse flow past obstacles in a rapidly rotating fluid. Journal of Fluid Mechanics, 32, 251-???.

Hogg, N.R. (1973) On the stratified Taylor column. Journal of Fluid Mechanics, 58, 517- 537.

Hogg, N.R. (1973b) The preconditioning phase of MEDOC 1969-II. Topographic effects, Deep Sea Research, 20, 449-459.

Howard, L. (1961) Note on a paper of John Miles, Journal of Fluid Mechanics, 10, 509-512.

Hufford, G. (1994) Parameterization of convection in a rotating stratified ocean; Comparison of numerical and laboratory experiments with theory. M.Phil. thesis, M.I.T.

Huppert, H.E., and K. Bryan (1976) Topographically generated eddies, Deep Sea Research, 23, 655-679.

Israeli, M. and S. Orszag (1981) Approximation of Radiation Boundary Conditions, Journal of Computational Physics, 41, 115-135.

Jacobs, S.J. (1964) The Taylor column problem. Journal of Fluid Mechanics, 58, 517537. 
Johnson, E.R. (1978) Trapped vortices in rotating flow, Journal of Fluid Mechanics, 86, 209-224.

Jones, H. and J. Marshall (1993) Convection with Rotation in a Neutral Ocean: A Study of Open-Ocean Deep Convection, Journal of Physical Oceanography, 23, 1009-1039.

Killworth, P.D. (1979) On "Chimney" Formations in the Ocean, Journal of Physical Oceanography, 9, 531-554.

Killworth, P.D. (1983) Deep convection in the world ocean, Reviews of Geophysics and Space Physics, 21, 1-26.

Klinger, B. A., J. Marshall, and U. Send (in press). Representation and parameterization of deep convective plumes by mixing. Submitted to Journal of Geophysical Research.

Legg, S. and J. Marshall (1993) A Heton Model of the Spreading Phase of Open-Ocean Deep Convection, Journal of Physical Oceanography, 23, 1040-1056.

Marshall, J., J. Whitehead and T. Yates (in press) Laboratory and Numerical Experiments in Oceanic Convection.

Martinson, D.G., P.D. Killworth, and A.L. Gordon (1981) A Convective Model for the Weddell Polynya, Journal of Physical Oceanography, 11, 466-488.

Maxworthy, T. and S. Narimousa (1994) Unsteady, Turbulent Convection into a Homogeneous, Rotating Fluid, with Oceanographic Applications, Journal of Physical Oceanography, 24, 865-887.

McCalpin, J. (1994) A Comparison of Second-Order and Fourth-Order Pressure Gradient Algorithms in a Sigma-Co-ordinate Ocean Model, International Journal for Numerical Methods in Fluids, 18, 361-383.

McCartney, M.S. (1975) Inertial Taylor columns on a beta plane, Journal of Fluid Mechanics, 68, 71-95.

Motoi, T., N. Ono, and M. Wakatsuchi (1987) A mechanism for the Formation of the Weddell Polynya in 1974, Journal of Physical Oceanography, 17, 2241-2247.

Muench, R.D. (1988) Relict Convective Features in the Weddell Sea, Deep Convection and Deep Water Formation in the Oceans, Chu and Gascard (Editors), 53-67.

Orlanski, I. (1976) A Simple Boundary Condition for Unbounded Hyperbolic Flows, Journal of Computational Physics, 21, 251-269. 
Ou, H.W. (1991) Some Effects of a Seamount on Oceanic Flows, Journal of Physical Oceanography, 21, 1835-1845.

Owens, W. and N. Hogg (1980) Oceanic observations of stratified Taylor columns near a bump, Deep Sea Research, 27a, 1029-1045.

Parkinson, C. (1983) On the Development and Cause of the Weddell Polynya in a Sea Ice Simulation, Journal of Physical Oceanography, 13, 501-511.

Pedlosky, J. (1987) Geophysical Fluid Dynamics, Springer-Verlag, 2nd edition, 710 pgs.

Smith, L. (1992) Numerical Simulations of Stratified Rotating Flow over Finite Amplitude Topography, Journal of Physical Oceanography, 22, 686-696.

Stone, P. (1972) A simplified radiative-dynamical model for the static stability of rotating atmospheres, Journal of Atmospheric Science, 29, 405-418.

Swallow, J.C. and G.F. Caston, (1973) The preconditioning phase of MEDOC 1969-I. Observations, Deep Sea Research, 20, 429-448.

Taylor, G.I. (1923) Experiments on the motion of solid bodies in rotating fluids. Proceedings of the Royal Society A, 104, 213-218.

Thompson, L. (1990) Flow over finite isolated topography, Ph.D. Thesis, Massachusetts Institute of Technology/Woods Hole Oceanographic Institution 91-05, 222 pp.

Thompson, L. (1993) Two-Layer Quasigeostrophic Flow over Finite Isolated Topography, Journal of Physical Oceanography, 23, 1297-1314.

Verron, J., and C. Le Provost (1985) A numerical study of quasi-geostrophic flow over isolated topography, Journal of Fluid Mechanics, 154, 231-252.

Verron, J. (1986) Topographic Eddies in Temporally Varying Oceanic Flows, Geophysical and Astrophysical Fluid Dynamics, 35, 257-276.

Visbeck, M., J. Marshall and H. Jones (in press) On the Dynamics of Convective "chimneys" in the Ocean, submitted to Journal of Physical Oceanography.

Zwally H., J. Comiso, C. Parkinson, W. Campbell, F. Carsey, and P. Gloerson (1983) Antarctic Sea Ice, 1973-1976: Satellite Passive-Microwave Observations, NASA SP459, 206 pgs. 